Tome 56, no 30. - Septembre 1949.

Travaux du Laboratoire de zoologie et d'anatomie comparée.

Université de Lausanne. Directeur: Professeur Robert Matтhey.

\title{
Les chromosomes des Chiroptères et des Insectivores
}

\author{
par \\ René Bover, lic. ès sciences.
}

(Avec 220 figures dans le texte et la planche 2.)

\section{SOMMAIRE}

\section{Avant-propos}

\section{Première partie: Les chromosomes des Chiroptères}

I. Historique . . . . . . . . . . . . . . . . . . . . . 375

II. Matériel . . . . . . . . . . . . . . . . . . . . 376

III. Le cycle spermatogénétique des Chiroptères . . . . . . 378

IV. Observations cytologiques . . . . . . . . . . . . . 379

1. Rhinolophidae:

Rhinolophus ferrum-equinum Schreb. . . . . . 379

Rhinolophus euryale Blasius . . . . . . . . . . 382

Rhinolophus hipposideros Bechst. . . . . . . . 386

2. Vespertilionidae:

Myotis myotis Borkhausen . . . . . . . . . . . 389

Myotis daubentonii Leisler . . . . . . . . . . . 393

Myotis mystacinus Leisler . . . . . . . . . . . 396

Myotis emarginatus Geoffroy . . . . . . . . . 397

Pipistrellus pipistrellus Schreb. . . . . . . . . . 398

Pipistrellus nathusii Keyserling et Blasius . . . 399

Miniopterus schreibersii Natterer . . . . . . . . 401

Plecotus auritus L. . . . . . . . . . . . . . 403

Barbastella barbastellus Schreb. . . . . . . . 407

3. Nycteridae:

Nycteris sp. . . . . . . . . . . . . . 409

Rev. Suisse de Zool., T. 56, 1949 . 25 
V. Discussion . . . . . . . . . . . . . 410

1. Les hétérochromosomes des Chiroptères . . . . 410

2. Liaison entre $\mathrm{X}$ et $\mathrm{Y}$ à la méiose . . . . . . . . . 413

3. Pré-réduction et post-réduction. . . . . . . . . 415

4. Hétérochromatie des chromosomes sexuels . . . . 415

5. Evolution chromosomique chez les Chiroptères . . 417

6. Systématique et chromosomes . . . . . . . . . . 419

Deuxième partie: Les chromosomes des Insectivores . . . . 420

I. Historique . . . . . . . . . . . . . . . . . . . . . 421

II. Matériel . . . . . . . . . . . . . . . . . . . . . . 421

III. Le cycle spermatogénétique des Insectivores . . . . . . 422

IV. Observations cytologiques . . . . . . . . . . . . . . 423

1. Erinaceidae:

Erinaceus europaeus L. . . . . . . . . . . . . 423

2. Talpidae:

Talpa europaea L. . . . . . . . . . . . . . 425

3. Soricidae:

Neomys fodiens Pallas . . . . . . . . . . . . 428

Crocidura russula Herm.. . . . . . . . . . . . 431

Sorex araneus L. . . . . . . . . . . . . . . . 437

V. Discussion . . . . . . . . . . . . . . 450

1. Les hétérochromosomes des Insectivores . . . . 451

2. Pré-réduction et post-réduction . . . . . . . . . 452

3. Hétérochromatie des chromosomes sexuels . . . . 452

4. Evolution chromosomique chez les Insectivores . . 453

Conclusions générales . . . . . . . . . . . . . 455

Résumé . . . . . . . . . . . . . . . . . 456

Auteurs cités. . . . . . . . . . . . . . . . 457

\section{AVANT-PROPOS}

Malgré le nombre déjà élevé de travaux parus dans de nombreux pays, la cytologie descriptive est loin d'avoir épuisé son sujet. En effet, si près d'un million d'espèces animales ont été décrites jusqu'à ce jour, une très faible fraction seulement a sollicité l'attention des cytologistes. Des ordres entiers sont encore pratiquement inexplorés, soit à cause des difficultés inhérentes à la capture du 
matériel, soit en raison d'obstacles techniques, tandis que d'autres groupes, d'un abord plus facile, ont donné lieu à de nombreux travaux. C'est ainsi que chez les Mammifères, on ignore presque tout des chromosomes des Monotrèmes, des Edentés, des Pinnipèdes, des Chiroptères et des Insectivores. En outre, de nombreuses contributions sont actuellement sans valeur, ayant été entreprises avec une technique périmée.

Ce travail vise à combler partiellement ces lacunes. Le sujet m'en fut proposé par le professeur R. Маттнеy en 1946. J'exprime d'emblée à mon maître ma très vive gratitude pour l'intérêt qu'il n'a cessé d'accorder à mes recherches et pour sa collaboration de tous les instants. Je remercie également toutes les personnes qui m'ont aidé à capturer les animaux nécessaires à cette étude, m'ont envoyé du matériel ou m'ont indiqué des stations où se trouvaient des colonies de Chiroptères: M. J.-L. Perrot m'a procuré des Noctules, M. Aellen m'a rapporté d'Afrique équatoriale des préparations de Nycteris, M. le professeur Brumpt et ses collaborateurs m'ont accueilli très aimablement à la station zoologique de Richelieu, MM. Heim de Balsac, Mislin, Dottrens, Hainard et LAURENT m'ont fourni de précieux renseignements. J'adresse encore mes remerciements à M. le $\mathrm{D}^{\mathrm{r}}$ REvilliod, qui a bien voulu confirmer quelques déterminations de Chiroptères et d'Insectivores, ainsi qu'à M. le Dr J. DE BEAU MONT, dont les conseils et les critiques m'ont été très utiles.

Au cours de cette étude, j'ai concentré mon attention sur trois points précis:

Tout d'abord, la détermination exacte du nombre et de la forme des chromosomes, dans les divisions spermatogoniales et réductionnelles. En second lieu, la forme et le comportement des hétérochromosomes. Enfin, comparant les résultats obtenus, j'ai cherché à les relier aux problèmes plus généraux de l'évolution et de la classification.

Ce travail est divisé en deux parties distinctes: la première est consacrée aux chromosomes des Chiroptères, la seconde à ceux des Insectivores. J'ai donné une plus grande importance à l'une des espèces étudiées, Sorex araneus, à cause de l'intérêt tout particulier que présente son trivalent sexuel. 


\section{TECHNIQUE}

J'utilise la technique de Minouchi (1928) telle que Matthey (1936) l'a décrite dans son travail sur les hétérochromosomes des Vertébrés. Toutes les pièces ont été fixées au Flemming sans acide acétique. Les coupes sont colorées à l'hématoxyline ferrique de

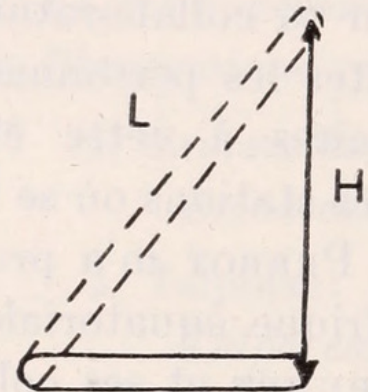

$P$

FIG. 1.

Schéma montrant la méthode graphique utilisée pour rétablir la longueur réelle d'un chromosome oblique. P: projection horizontale du chromosome, dessinée à la chambre claire. $\mathrm{H}$ : différence de niveau entre les deux extrémités. L: longueur réelle.

Heidenhain, à la fuchsine basique de Feulgen, ou à la triple coloration de Flemming. Les dessins ont été faits à la chambre claire, avec la combinaison optique suivante: obj. imm. Leitz 1/12, ocul. 20, tube tiré à 156. Le croquis, agrandi directement à 6550 fois, à l'aide d'un appareil que j'ai mis au point et dont je donne la description ailleurs, est terminé au crayon, puis à l'encre de Chine. Les caryogrammes sont élaborés à partir de photocopies des dessins: je découpe les chromosomes et je les arrange par paires, en tenant compte à la fois de leur taille et de leur forme, et en contròlant la séria-

tion par l'examen direct de la figure au microscope. Lorsqu'un chromosome n'est pas horizontal, je calcule sa longueur réelle graphiquement, en mesurant, à l'aide de la vis micrométrique graduée, la différence de niveau entre ses deux extrémités. Il est évident que cette méthode n'est valable que si le chromosome en question est à peu près rectiligne (fig. 1).

Pour chaque espèce, j'ai donné une ou plusieurs microphotographies, que le lecteur trouvera à la planche II. 
PREMIẼRE PARTIE

\section{LES CHROMOSOMES DES CHIROPTÈRES}

\section{HISTORIQUE}

Les travaux consacrés aux chromosomes des Chiroptères sont peu nombreux. Les difficultés matérielles des récoltes peuvent expliquer que la plupart des cytologistes ne se soient pas intéressés à ce groupe.

Van der Stricht (1910), étudiant l'ovogenèse de Vesperugo noctula, trouve 9 à 10 éléments dans les divisions haploïdes.

Aтнias (1912) décrit 16 bivalents chez la femelle de Rhinolophus hipposideros, et 15 à 24 chez Vesperugo serotinus.

JoRDAN (1912) compte environ 24 chromosomes et suppose une digamétie $X O$ chez le mâle d'une espèce qui n'est pas désignée exactement.

Les données de Hance (1917) sont plus vraisemblables, bien que l'auteur considère le nombre diploïde de 40 chromosomes comme approximatif. Maîheureusement, l'espèce n'est pas nommée.

En revanche, le travail de Painter (1925) est d'un plus grand intérêt, puisque Nyctinomus mexicanus, étudié par l'auteur américain, est le seul représentant de la famille des Molossidae dont la formule chromosomique soit actuellement connue. PaInter, qui n'a observé que des divisions diploïdes, décrit 48 chromosomes et une digamétie ô de type $X Y$ chez cette Chauve-souris.

Tout récemment enfin, Makino (1948) a étudié deux espèces asiatiques: chez Pteropus dasymallus inopinatus Kuroda, un Mégachiroptère provenant des îles Riou-Kiou, le cytologiste japonais compte 38 chromiosomes, la plupart métacentriques, dont une paire d'hétérochromosomes $X Y$; chez Rhinolophus ferrum-equinum nippon Temminck, une sous-espèce de Rhinolophus ferrum-equinum Schreber que j'ai étudié, Makino trouve 29 bivalents dans les spermatocytes de premier ordre. Ces résultats concordent entièrement avec ceux que j'ai obtenus chez l'espèce européenne. 
Mentionnons pour terminer la note que j'ai publiée en collaboration avec $R$. Matthey, donnant les résultats acquis à la fin de l'année 1947 (Matтhey et Bovey, 1948).

Pour tout ce qui concerne les travaux sur les chromosomes des Mammifères, je renvoie le lecteur à l'ouvrage de Matthey: Les chromosomes des Vertébrés, (Rouge-Lausanne). Il y trouvera d'ailleurs une partie des figures du présent travail.

\section{MATÉRIEL}

La recherche du matériel a été souvent ardue et décevante. En effet, si les Chauves-souris sont faciles à trouver durant la mauvaise saison dans les grottes, les souterrains, les trous de murs ou les clochers, où elles reviennent régulièrement hiverner chaque année, il n'en est pas de même en été. A cette saison, la seule qui soit favorable pour une étude chromosomique, la plupart des espèces se dispersent dans les forêts, et les mâles vivent en général solitaires ou en petits groupes, en des gîtes qui ne sont pas fixes. Les femelles, les jeunes de l'année et quelques mâles plus âgés forment des colonies plus grandes, où il est facile de retrouver la même espèce chaque année. Mais ces colonies ne fournissent que très peu de mâles adultes.

J'ai essayé de fouiller les trous d'arbres, mais, dans nos forêts, les arbres creux sont rares, et la plupart des cavités sont vides. En fait, cette méthode ne m'a donné aucun résultat. Restaient les toits de vieux châteaux, de maisons, d'églises: j'en ai visité plusieurs centaines, trouvant presque toujours Rhinolophus hipposideros, Myotis myotis, et, plus rarement, Plecotus auritus. Peu à peu, de nombreux amis et collègues me signalèrent des localités propices ou m'envoyèrent du matériel, et les prospections devinrent plus efficaces grâce aux expériences acquises. .Il m'a fallu cependant parcourir plusieurs milliers de kilomètres pour recueillir le matériel de treize espèces européennes, dont dix furent capturées en Suisse romande et trois en France. Ajoutons encore deux espèces africaines dont le matériel a été obligeamment fixé par un ami, ce qui porte à quinze le nombre total des espèces que j'ai eues sous les yeux. Malheureusement, deux espèces, Nyctalus noctula et Epomophorus sp. 
ne m’ont donné aucun résultat, la première ayant été fixée trop tard, la seconde dans de mauvaises conditions. Chez trois autres espèces, Myotis mystacinus, M. emarginatus et Nycteris sp., les observations sont incomplètes, et je n'en rends compte qu'à titre provisoire.

Au cours de mes expéditions, j'ai pu faire de nombreuses observations faunistiques et écologiques qui seront publiées prochainement.

\section{LISTE DES ESPÈcES ÉTUdiéES ${ }^{1}$.}

\begin{tabular}{|c|c|c|c|}
\hline Espèce & Adultes & Jeunes & Localités \\
\hline $\begin{array}{c}\text { Rhinolophidae } \\
\text { Rhinolophus ferrum-equinum }\end{array}$ & & & \\
\hline Schreb. . . & 2 & 4 & $\begin{array}{l}\text { Richelieu (Indre-et-L.) } \\
\text { Taizé (Saône-et-Loire) }\end{array}$ \\
\hline euryale Blasius . . & 1 & 4 & St-Paterne (Indre-et-L.) \\
\hline " $\quad$ hipposideros & 13 & 10 & \\
\hline Vespertilionidae & 13 & 10 & Lausame el enviroms \\
\hline Myotis myotis Borkhausen . . & 4 & 9 & Lutry, Chillon, Oron \\
\hline " daubentonii Leisler. . . & 3 & 2 & Genève. \\
\hline " mystacinus Leisler . . & 2 & 6 & Romanel s/Lausanne \\
\hline - " emarginatus Geoffroy. . & 一 & 4 & Richelieu (Indre-et-L.) \\
\hline $\begin{array}{c}\text { Pipistrellus pipistrellus } \text { Schreber } \\
\text { „) } \\
\text { nathusii Keyserling }\end{array}$ & 3 & 1 & Cheseaux, Oron, Allaman \\
\hline et Blas. . . . & 2 & - & Cheseaux, Allaman \\
\hline Miniopterus schreibersii Natt. . & 11 & 1 & $\begin{array}{l}\text { Chambrelien s/Neuchâtel, } \\
\text { Saillon (Valais) }\end{array}$ \\
\hline Plecotus auritus L. . . . . . . & 4 & 1 & $\begin{array}{l}\text { Salins, Neuchâtel, Mon- } \\
\text { theron s/Lausanne }\end{array}$ \\
\hline Barbastella barbastellus Schreber & 5 & 1 & Apples, Rolle \\
\hline $\begin{array}{c}\text { Nyctalus noctula Schreber } * \\
\text { Nycte RIDAE }\end{array}$ & 2 & - & Allaman, Epalinges \\
\hline Nycteris sp. $\underset{\text { PTEROPIDAE }}{.} \cdot \cdot \cdot \cdots \cdot$ & 1 & - & Tibati (Cameroun) \\
\hline Epomophorus sp. *. . . . . & 1 & - & Tibati (Cameroun) \\
\hline
\end{tabular}

Le nombre relativement grand d'individus sacrifiés illustre bien la difficulté de la fixation des chromosomes chez les Mammifères. Les échecs proviennent aussi, il est vrai, de fixations faites à une époque défavorable. En moyenne, un individu sur cinq a livré de bons résultats, et de toutes les espèces étudiées, c'est Rhinolophus hipposideros qui m'a donné le plus de difficultés.

1 Les espèces marquées du signe * n’ont pas donné de figures numérables. 


\section{LE CYCLE SPERMATOGENÉTIQUE DES CHIROPTÈRES}

On sait, depuis les travaux de Courrier (1927) que la spermatogenèse se déroule en été chez les Chiroptères. Comme mes observations sont en général en accord avec celles de cet auteur, ce chapitre sera bref.

En hiver, durant le sommeil léthargique, les tubes séminifères sont très petits, et ne renferment, à part les cellules de Sertoli, que des spermatogonies et parfois quelques spermatozoïdes provenant de la saison précédente. Toutefois l'activité mitotique n'est jamais totalement suspendue: j’ai en effet trouvé des mitoses spermatogoniales, assez rares il est vrai, à la fin de décembre, chez un Oreillard. Ce n'est cependant qu'au début de l'été, en mai ou en juin, que commence, chez les Chiroptères adultes de notre région, l'activité mitotique intense qui caractérise la reprise de la spermatogenèse. A ce moment, on trouve d'une part des métaphases isolées et rares, de grande taille, qui lorsqu'elles sont bien fixées donnent des images excellentes; d'autre part des mitoses nombreuses et groupées, mais de petite taille, et qui restent dans la plupart des cas indéchiffrables: ce sont les cellules-filles des ultimes générations goniales. Au moment où apparaissent les premières métaphases auxocytaires, les mitoses spermatogoniales sont déjà très rares. Plus tard, on n'en trouve que très difficilement, du moins chez les adultes de plus de deux ans. Du 15 juillet au 15 août, on peut observer tous les stades de la spermatogenèse. Dès le début de septembre, les tubes séminifères ne montrent plus aucune division, et les spermatogonies qui restent forment, avec les cellules de Sertoli, une mince strate entourant la masse spermatique. Quelques-unes entrent en division en automne déjà, et cette activité mitotique discrète reconstitue peu à peu le stock de gonies.

Chez l'adulte, les conditions météorologiques influencent le cycle spermatogénétique. Ainsi, en 1947, année dont l'été fut chaud et peu pluvieux, tous les individus capturés à la fin d'août ou au début de septembre furent inutilisables, leur spermatogenèse étant déjà complètement terminée. L'année suivante, le printemps et le début de l'été furent relativement froids et pluvieux; je fus très surpris 
de voir des noyaux pachytènes et de nombreuses divisions réductionnelles dans des testicules fixés au milieu de septembre.

L'âge est le second facteur dont j'ai pu déceler l'influence sur le déroulement de la spermatogenèse. Les jeunes naissent au mois de mai ou de juin, peu après le rassemblement des femelles en colonies d'élevage (Wochenstuben). En quelques semaines, ils atteignent la taille des adultes, mais ils ne font pas de spermatogenèse durant la première année, bien que leurs testicules montrent parfois de belles métaphases spermatogoniales. On trouve encore dans les colonies d'élevage, outre les femelles et les jeunes de l'année, quelques rares mâles adultes, dont la dentition intacte et l'ossification incomplète montrent clairement le caractère juvénile. Agés probablement d'une année, ils ont rejoint la colonie où ils sont nés l'année précédente, tandis que les autres mâles vivent isolés ou par petits groupes. Or, j'ai constaté sans exception que leur spermatogenèse, la première de leur existence, se produit avec un retard de plus d'un mois par rapport au rythme normal du mâle âgé. Ces faits ont été vérifiés chez trois espèces: Barbastella barbastellus, Pipistrellus pipistrellus, Rhinolophus ferrum-equinum.

Les deux espèces africaines que j'ai pu obtenir, Epomophorus sp. et Nycteris sp., dont les testicules ont été fixés en juin, présentent à cette époque tous les stades de la spermatogenèse.

\section{ORSERVATIONS CYTOLOGIQUES}

Autant que possible, j'ai observé les stades suivants: division spermatogoniale, première et seconde division de maturation, avec anaphase de profil montrant le comportement des hétérochromosomes. Une étude plus détaillée des stades de la méiose m'a donné l'occasion de préciser, chez Myotis myotis, l'évolution du complexe sexuel $X Y$.

\section{Famille des Rhinolophidae.}

Rhinolophus ferrum-equinum Schreber.

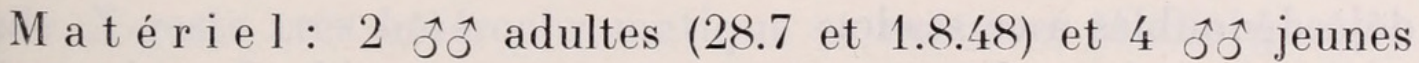
(28.7.48); ces derniers, âgés de trois mois environ, n'ont donné aucun résultat. 
O b s e r v a t i o n s : Les divisions spermatogoniales (fig. 2 et 3 ) attestent d'emblée le nombre élevé de chromosomes, 58 chez cette espèce. La plupart d'entre eux sont en forme de bâtonnets plus ou moins longs, à centromère subterminal. Sept éléments, parmi
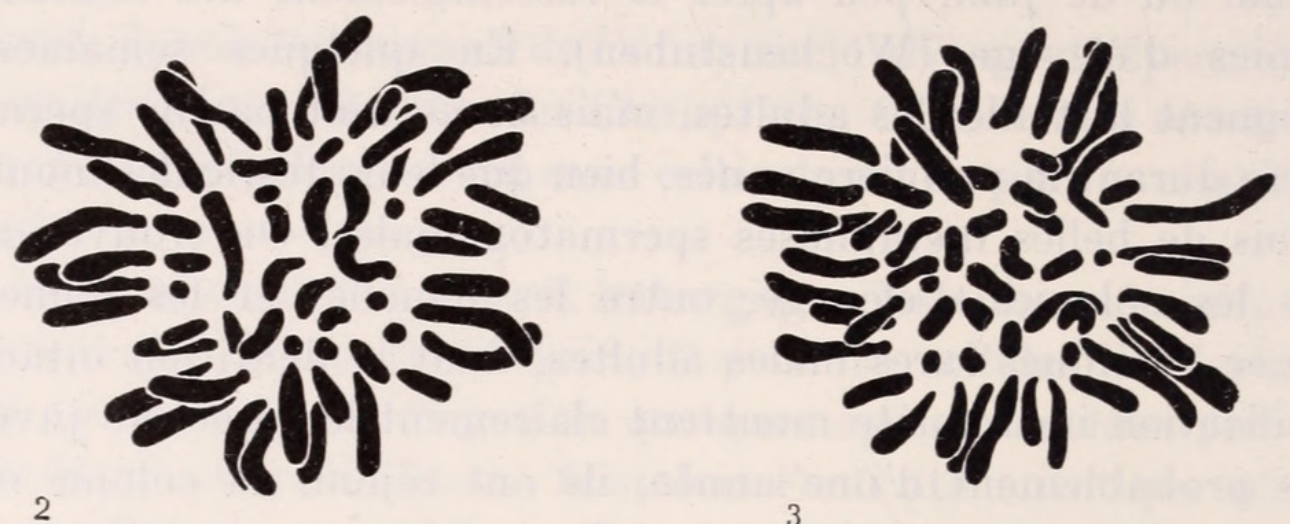

FIG. 2 et 3. - Rhinolophus ferrum-equinum Schr. Métaphases spermatogoniales. Hématoxyline $(\times 4000)$.

lesquels figure le chromosome $Y$, sont punctiformes. L'appariement des chromosomes (fig. 4) met en évidence les chromosomes sexuels $X$ et $Y$, de tailles très différentes. $X$ est un métacentrique à bras

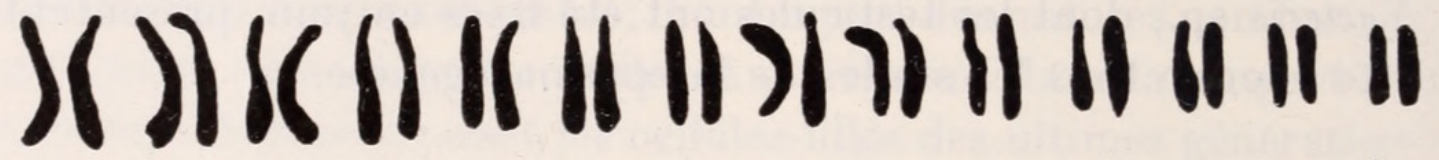

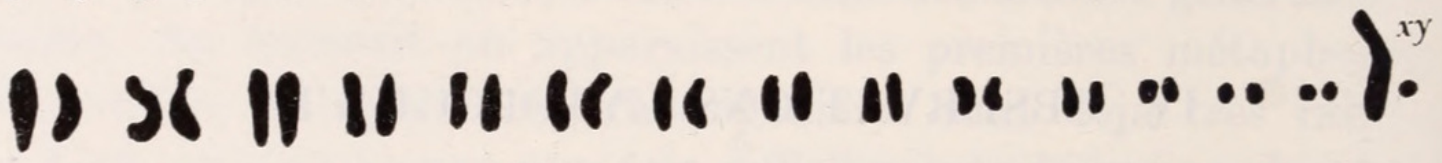 \\ FIG. 4. - Rhinolophus ferrum-equinum Schr. Chromosomes spermatogoniaux $(\times 4000)$.}

inégaux, qui n'est pas hétérochromatique dans les divisions goniales. Quant à $Y$, c'est l'un des plus petits éléments punctiformes.

Les premières divisions réductionnelles (fig. 5-8) sont d'une qualité exceptionnelle, et j'en ai examiné plusieurs dizaines. Toutes montrent clairement 29 bivalents, répartis très régulièrement en une plaque équatoriale petite et dense. La plupart d'entre eux forment des anneaux, parfois ouverts vers l'extérieur, l'un des chiasmas s'étant déjà résolu. Les plus petits bivalents sont souvent déjà dédoublés à ce stade.

Le complexe $X Y$ est aisément reconnaissable dans les métaphases auxocytaires; d'une part parce que son axe est déjà parallèle 
au fuseau bien avant l'anaphase, et que sa hauteur est supérieure à celle des bivalents autosomiques; et d'autre part grâce à sa forme particulière. Les métaphases fortement différenciées révèlent une hétérochromatie positive persistante, qui permet de suivre ses composants $X$ et $Y$ jusqu'à la fin de la deuxième division de maturation.

Les hétérochromosomes occupent une position périphérique (fig. 5 et 6 ) ou centrale (fig. 7 et 8). La séparation des éléments $X$
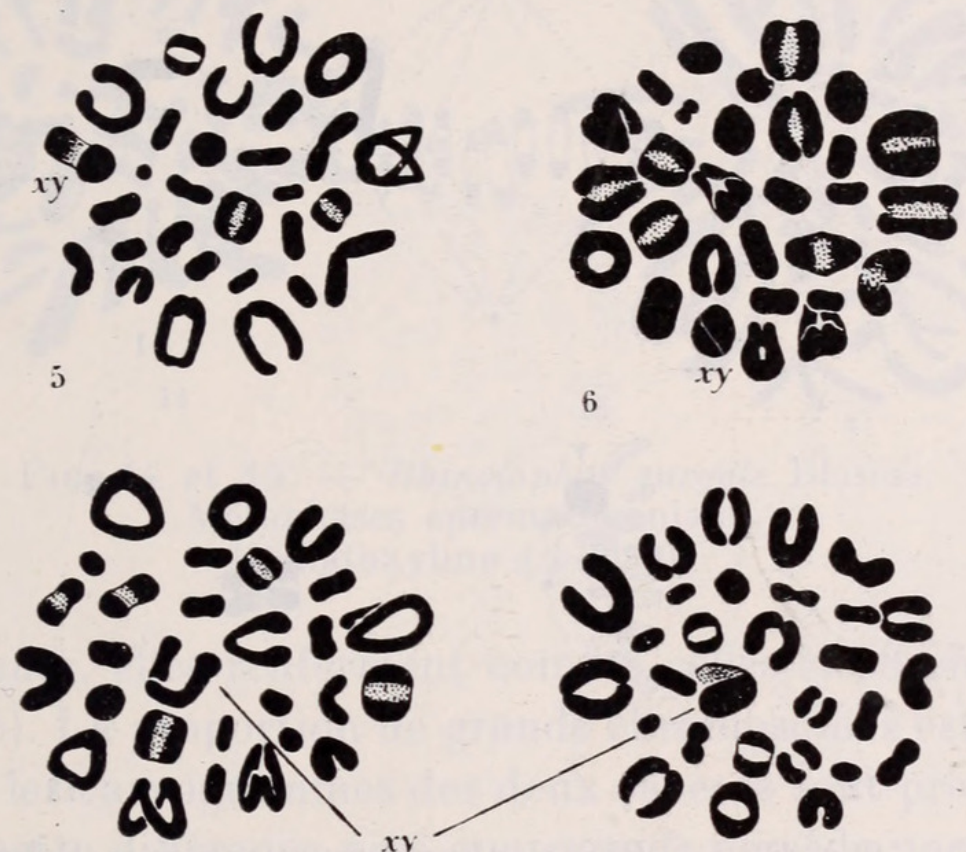

7

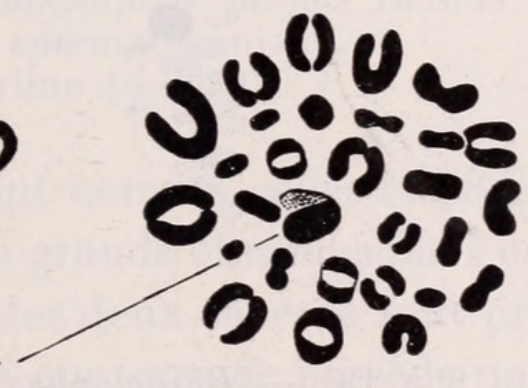

8

Fig. 5 à-8. - Rhinolophus ferrum-equinum Schr. Métaphases auxocytaires; $x y=$ hétérochromosomes. Hématoxyline $(\times 4000)$

et $Y$ est plus précoce que celle des dyades autosomiques (fig. 9), et au début de l'anaphase déjà, le chromosome $Y$ est à l'extrémité terminale du grand bras de l' $X$, prolongeant ce dernier qui est maintenant perpendiculaire à la plaque équatoriale. Quant au bras court de l' $X$, il est en général tourné vers l'extérieur du fuseau, obliquement. L'ensemble figure une lettre i, qui attire l'attention lorsqu'on examine une métaphase de profil, surtout si les autosomes sont peu colorés (fig. 10). Malgré la séparation précoce des chromosomes $X$ et $Y$ (fig. 11), leur ascension polaire se fait en même temps que celle des autosomes. A la fin de l'anaphase, la dyade $X$ reste très hétérochromatique et massive, tandis que les autosomes prennent un aspect flou. Les deux chromatides de l' $X$ apparaissent souvent écartées dans le grand bras (fig. 12). 
Durant l'intercinèse, les noyaux sont pâles, et les autosomes invisibles à l'intérieur. En revanche, les hétérochromosomes, appliqués contre la membrane nucléaire, restent fortement colorés. Il est facile de reconnaître les deux cellules qui sont issues du même spermatocyte I: leurs noyaux sont en effet très rapprochés, et la membrane qui les sépare est souvent marquée par la présence de la
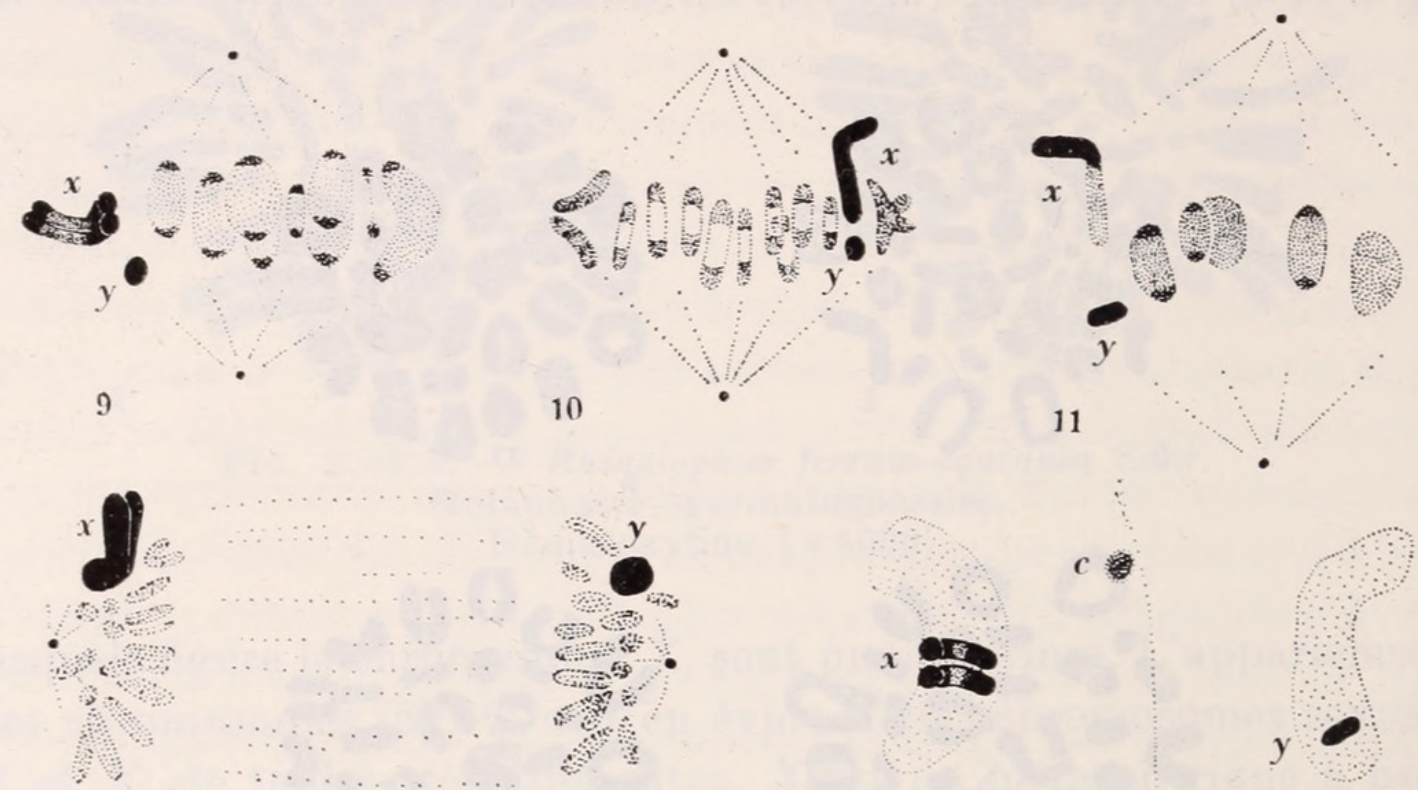

12

13

Fig. 9 à 13. - Rhinolophus ferrum-equinum Schr.

FIG. 9 à 11: Métaphases auxocytaires vues de profil, et montrant les hétérochromosomes $x$ et $y$. - Fig. 12: Fin d'anaphase de première cinèse. - FIg. 13: Télophase. $c=$ corps chromatoïde. Hématoxyline $(\times 4000)$.

plaque intermédiaire. Dans ces paires de spermatocytes II, j'ai toujours trouvé $X$ dans un noyau, $Y$ dans l'autre: leur ségrégation est donc pré-réductionnelle (fig. 13).

Je n'ai pu numérer avec certitude les secondes cinèses de maturation, mais j'ai pu constater par contre que l'évolution des hétérochromosomes est en tout point semblable à celle que je décris chez Myotis myotis (cf. p. 389).

\section{Rhinolophus euryale Blasius.}

M a t é r i e l: 1 ô adulte et 4 jeunes $\widehat{o} \widehat{o}(28.7 .48)$. Ces individus, provenant de Saint-Paterne (Indre-et-Loire, France), appartiennent à la sous-espèce atlanticus décrite par Andersen et Matschie 
(1904), et ont été capturés dans la grotte où Trouessart les avait signalés il y a soixante-dix ans déjà. Les jeunes n'ont pas donné de figure utilisable.

$\mathrm{O}$ b s e r vations: Les divisions spermatogoniales sont fort rares dans mon matériel. Très semblables à celles de Rhinolophus.

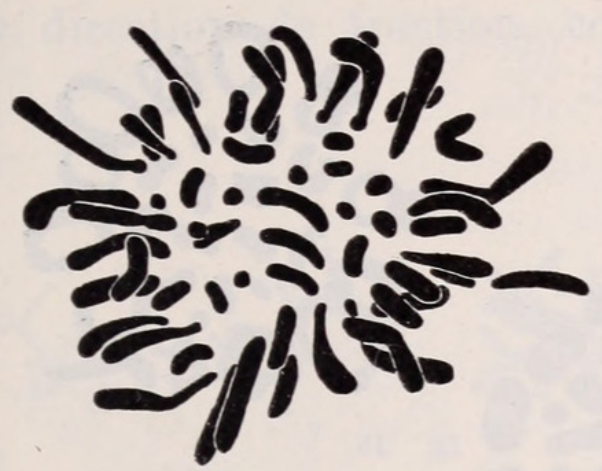

14

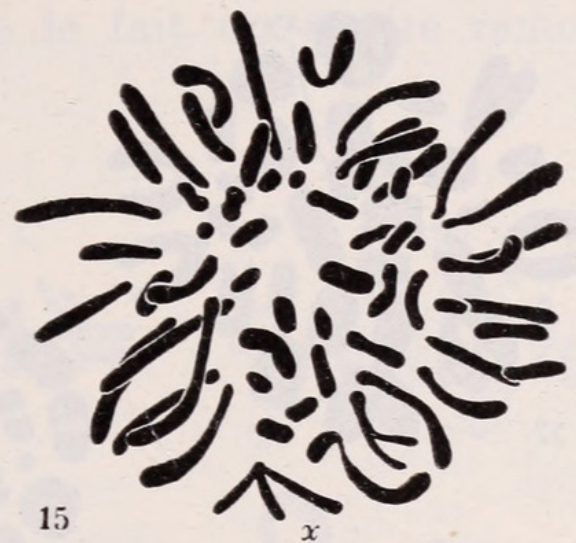

15

FIG. 14 et 15. - Rhinolophus euryale Blasius. Métaphases spermatogoniales. Hématoxyline $(\times 4000)$.

ferrum-equinum, elles renferment comme celles-ci 58 chromosomes (fig. 14 et 15). La proportion de grands chromosomes est à peu près la même, et les caryogrammes des deux espèces sont presque superposables, à cette différence près que euryale possède une paire d'au-

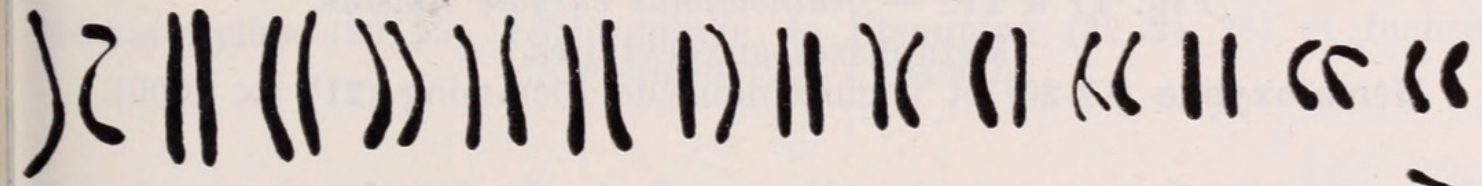

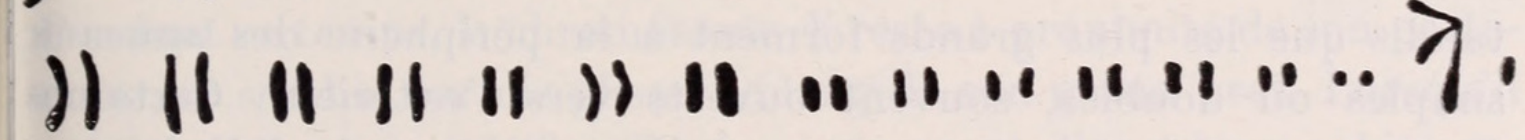

FIG. 16. - Rhinolophus euryale Blasius.

Chromosomes spermatogoniaux $(\times 4000)$.

tosomes métacentriques, de taille moyenne, tandis que ferrum equinum n'a probablement que des acrocentriques, à part l' $X$. Parmi les plus petits éléments, on peut dénombrer six ou sept chromosomes punctiformes, dont l' $Y$. Un des composants de la figure 15 est nettement hétérochromatique, et ses deux chromatides divergent largement dans l'un des bras: il s'agit probablement de l'hétérochromosome $X$, comme le montre la sériation du caryogramme (fig. 16). 
Les premières divisions de maturation (fig. 17-21) confirment le nombre diploïde observé dans les divisions goniales: elles montrent en effet 29 bivalents, rangés en une plaque équatoriale un peu plus grande, en moyenne, que celle de Rhinolophus ferrum-equinum, et disposés très régulièrement, les plus petits occupant le centre,
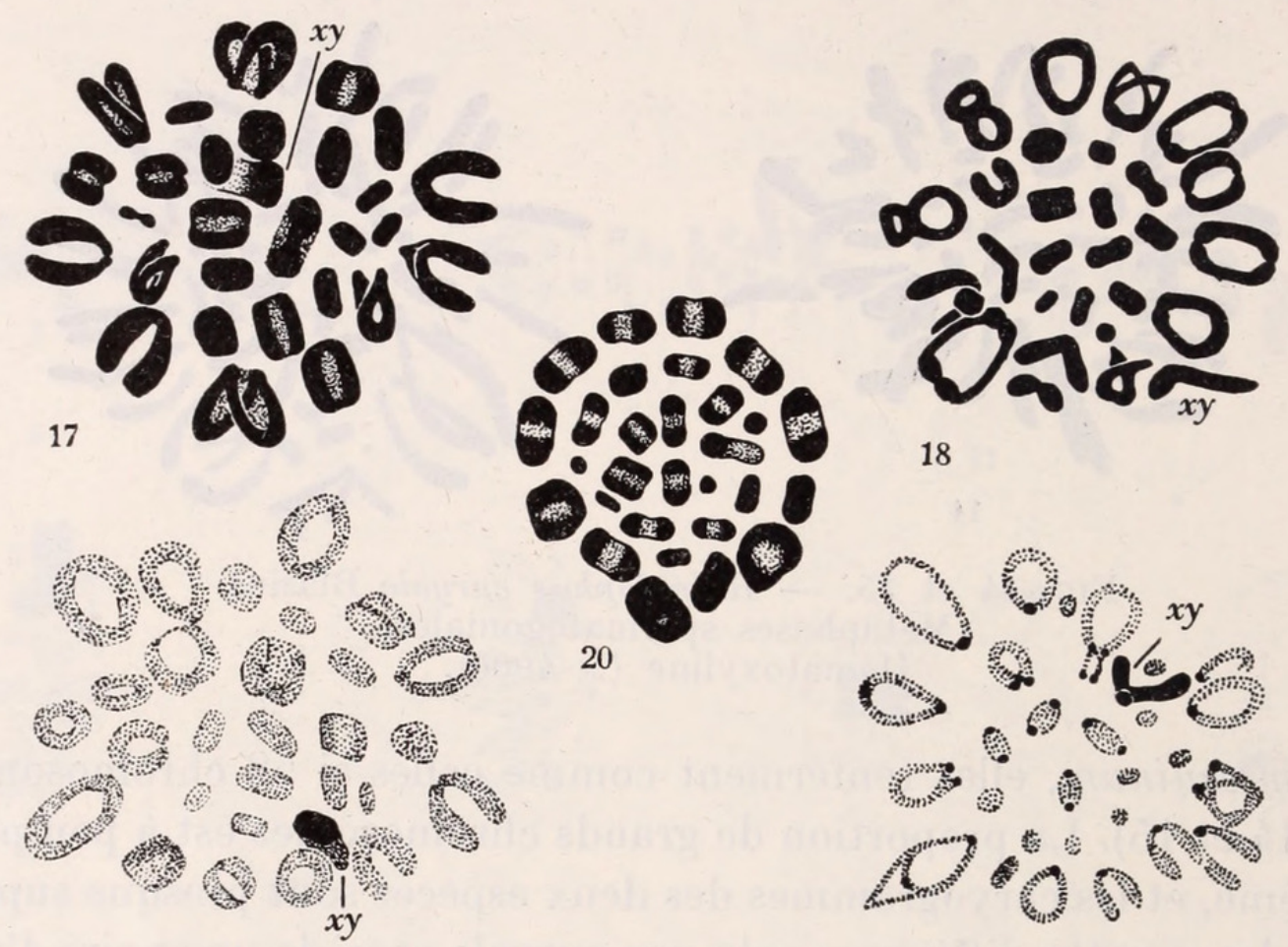

18

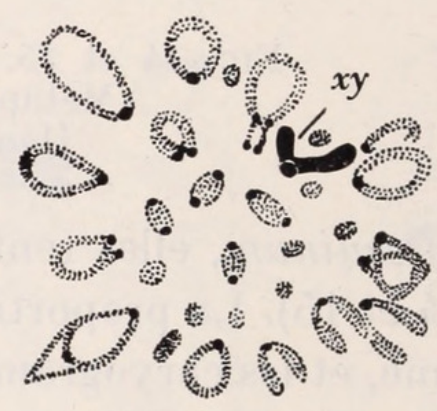

10

2]

FIG. 17 à 21. - Rhinolophus euryale Blasius.

Métaphases auxocytaires.

Hématoxyline (17-20) et trichromique de Flemming $(21)(\times 4000)$.

tandis que les plus grands forment à la périphérie des anneaux simples ou doubles, souvent ouverts vers l'extérieur. Certaines figures, peu colorées, mettent en évidence le couple $X Y$, hétérochromatique: généralement vertical, - l' $Y$ étant alors à l'extrémité du bras long de l' $X$-, il apparaît quelquefois parallèle au plan équatorial (fig. 18 et 21), et dans ce cas, l' $Y$ est proche de la région centromérique de l' $X$. Ces deux dispositions sont en apparence contradictoires, mais les métaphases vues de profil (fig. 22-27) donnent la clé du problème: au début de la métaphase, l' $X$ se place dans le plan équatorial, et l' $Y$ se dispose en parasynapsis (fig. 18, 21, 23). Ce stade semble très fugace, et difficile à observer, car à ce moment la paire $X Y$ ressemble à une tétrade autosomique. Très tôt, avant que les autres bivalents se soient divisés, les deux 
chromosomęs sexuels se séparent (fig. 23; voir aussi fig. $9, R h$. ferrum-eq.): $X$ tourne à angle droit, et son grand bras se place alors parallèlement à l'axe du fuseau. Cette rotation est probablement due à la résistance de la liaison entre $X$ et $Y$, - quelle que soit la nature de celle-ci, - et à la viscosité du cytoplasme: le chromosome $X$, tiré au niveau de son centromère, s'oriente parallèlement à la direction de traction, comme le fait un navire remorqué.

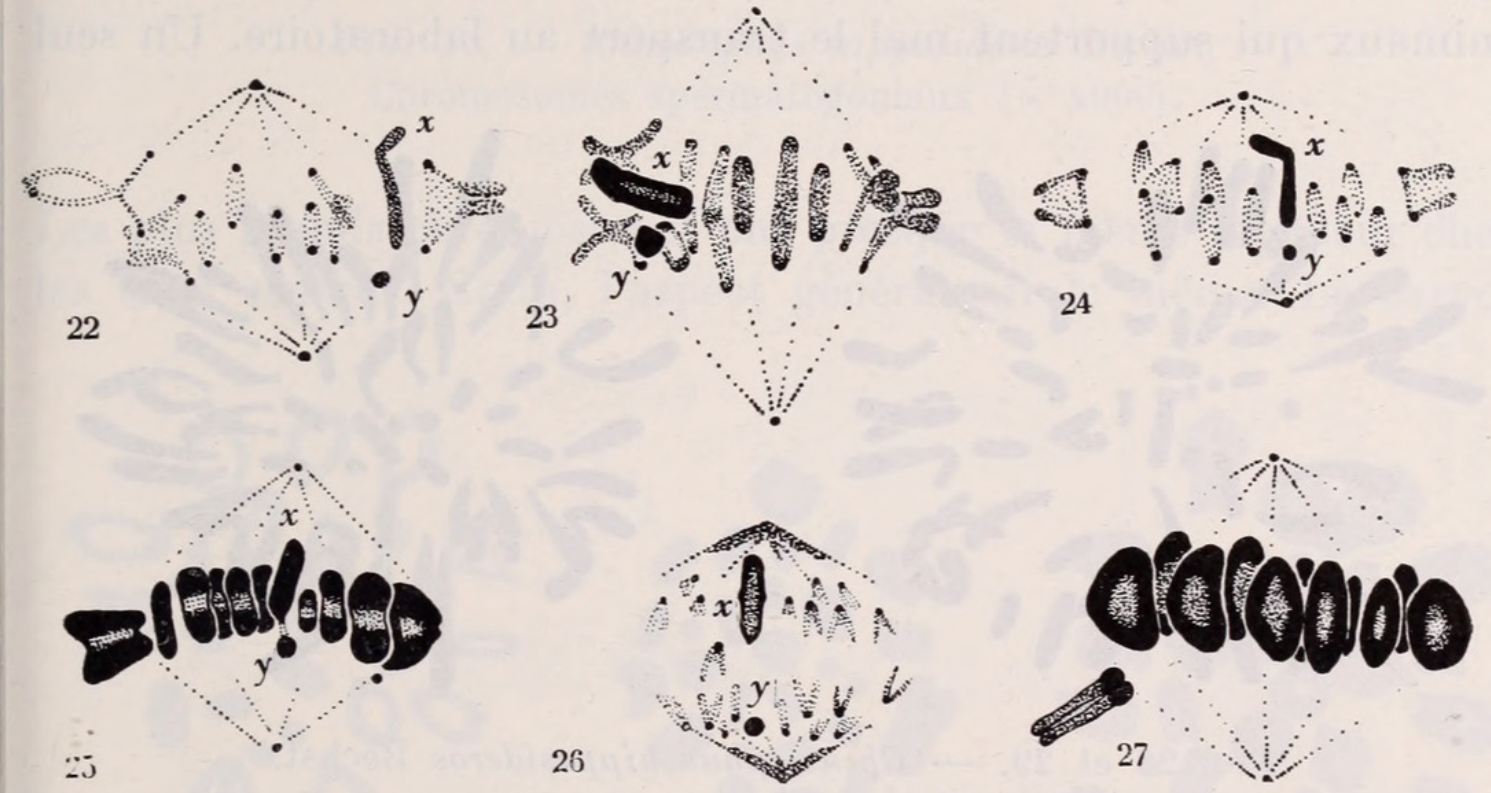

FIG. 22 à 27. - Rhinolophus euryale Blasius.

Métaphases et anaphases auxocytaires vues de profil, et montrant les hétérochromosomes $x$ et $y$.

Hématoxyline $(23,25)$, trichromique de Flemming $(22,24,26)$ et fuchsine de Feulgen $(27)(\times 4000)$.

Pendant ce temps, le chromosome $Y$ reste à proximité de son partenaire, et semble parfois glisser le long de son grand bras. Lorsqu'il atteint son extrémité (fig. 25), le mouvement d'anaphase subit probablement un temps d'arrêt, car ce stade typique est extrêmement fréquent. Parfois, une légère traînée plus foncée que le cytoplasme relie les deux constituants du couple $X Y$, et donne l'impression qu'ils sont attachés bout à bout. Il s'agit probablement d'un artifice, car l'apparition de cette traînée n'est pas constante, et dépend de la coloration.

Je n'ai trouvé qu'une seule figure qui puisse indiquer une postréduction des hétérochromosomes (fig. 27), et son interprétation est même sujette à caution. On peut donc considérer que la préréduction est normale chez Rhinolophus euryale. 
Les secondes divisions de maturation, fort rares dans mon matériel, n'ont pas été dessinées. L'évolution ultérieure des hétérochromosomes se poursuit comme chez Myotis myotis.

\section{Rhinolophus hipposideros Bechstein.}

M a t ér i e l: $13 \hat{\jmath} \widehat{o}$ adultes, 10 jeunes $\hat{o} \hat{o}$. La fixation s'est révélée difficile, probablement en raison de la fragilité de ces animaux qui supportent mal le transport au laboratoire. Un seul
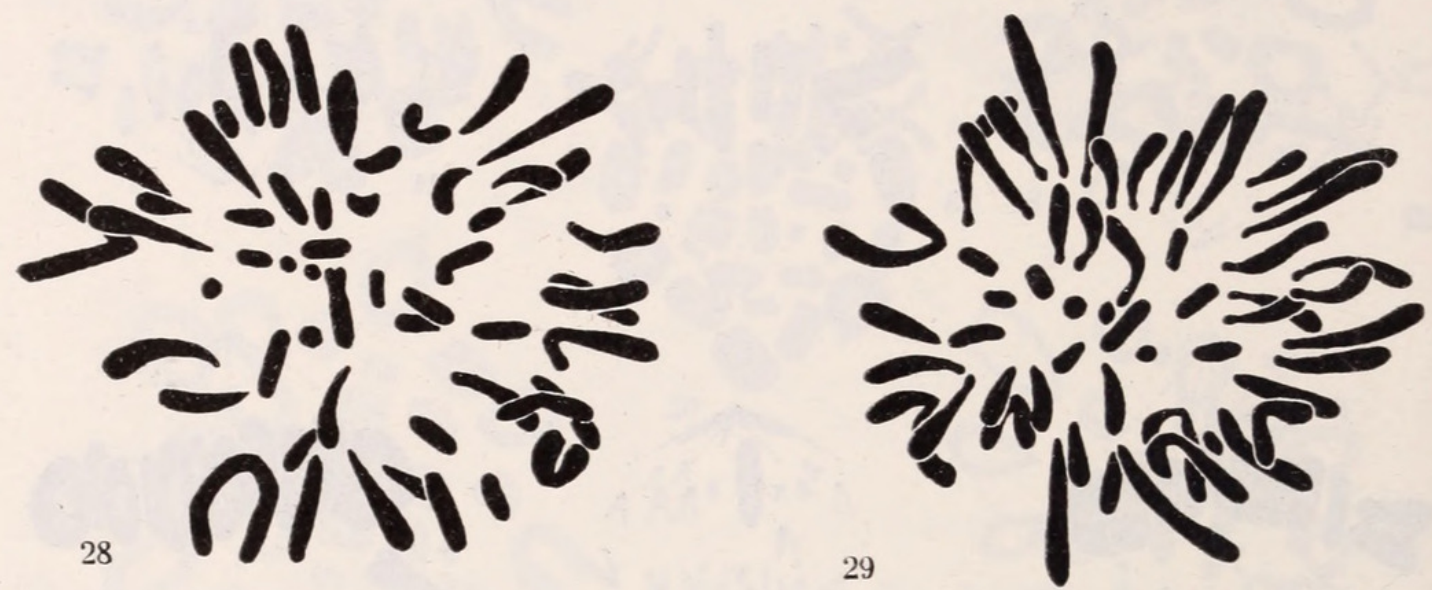

FIG. 28 et 29. - Rhinolophus hipposideros Bechst.

Métaphases spermatogoniales.

Fuchsine de Feulgen $(\times \mathbf{4 0 0 0})$.

des jeunes m'a donné de bonnes cinèses spermatogoniales. Chez les adultes, les chromosomes étaient souvent empâtés, ou se coloraient mal.

Observations: Cette espèce, plus petite que les deux précédentes, s'en distingue par des caractères morphologiques très nets. Les données cytologiques, elles aussi, éloignent $R h$. hipposideros des espèces plus grandes ferrum-equinum et euryale. Ces dernières, on l'a vu, ont toutes deux 58 chromosomes; Rh. hipposideros n'en possède que 54, mais compte dans son assortiment trois paires d'éléments en $\mathrm{V}$, ce qui lui donne un nombre fondamental (nombre total de bras chromosomiques $=N . F$.) égal à celui de Rh. euryale (61). L'examen cytologique des Rhinolophidae vérifie donc assez exactement la loi de Roвertson (1916).

L'observation des métaphases spermatogoniales (fig. 28 et 29) montre bien la parenté très étroite qui unit hipposideros aux deux 
autres espèces du genre. Les éléments punctiformes sont plus rares, mais on trouve à peu près le même nombre de bâtonnets courts.

\section{7|||||||||||||||| $\mid)|\alpha| \mid$

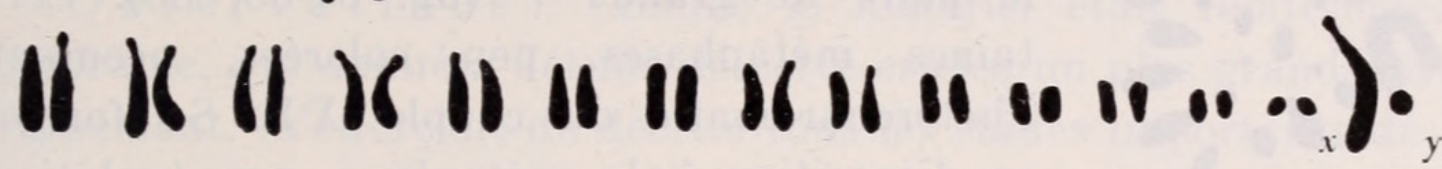

FIG. 30. - Rhinolophus hipposideros Bechst.

Chromosomes spermatogoniaux $(\times 4000)$.

Les plus grands chromosomes ont presque la même longueur chez les trois espèces. Enfin, l'aspect général est le même. Le caryo-

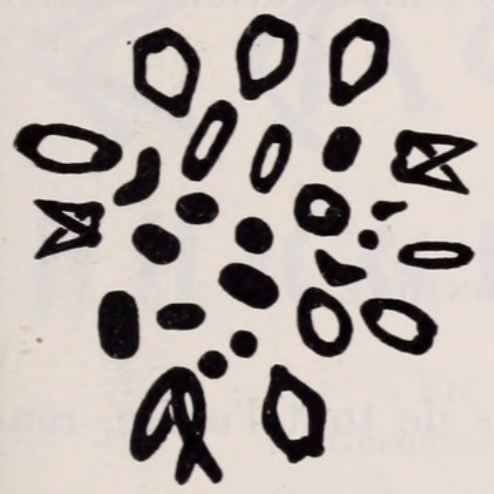

31

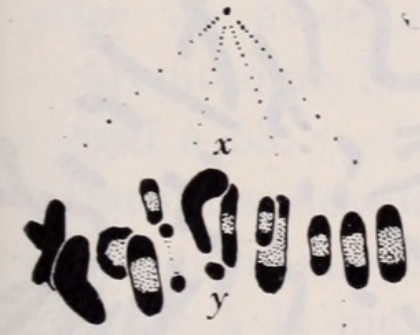

34

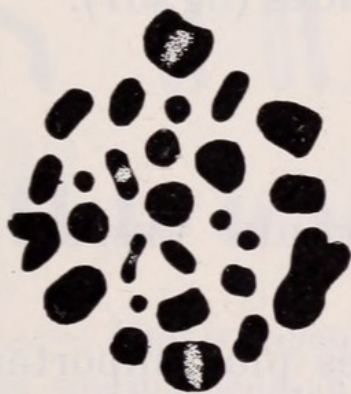

32

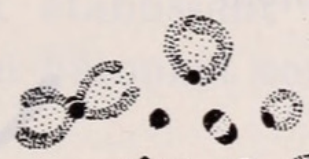

-

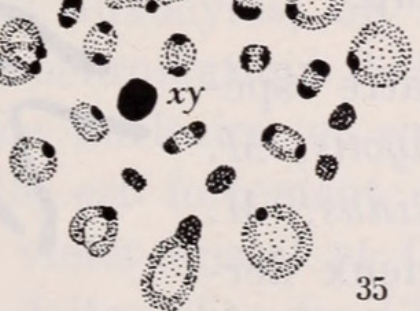

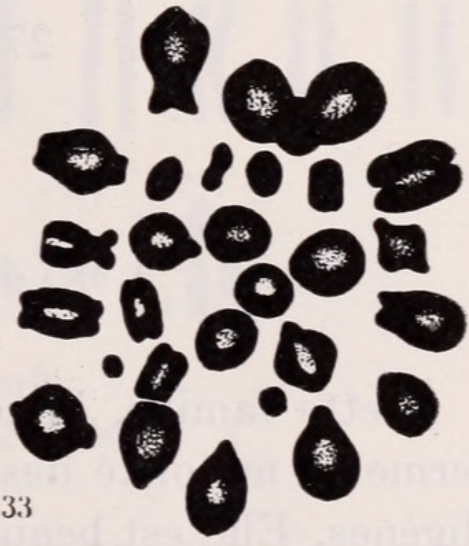

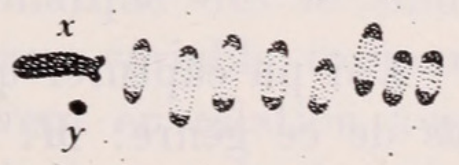

$36 \div$

FIG. 31 à 36. - Rhinolophus hipposideros Bechst.

Métaphases auxocytaires, en vue polaire et de profil.

Hématoxyline, sauf la fig. 33, colorée Feulgen $(\times 4000)$.

gramme (fig. 30) indique une paire $X Y$ formée d'éléments très dissemblables: $X$ est acrocentrique et $Y$ punctiforme.

Rev. Suisse de Zool., T. 56, 1949. 
Après les stades de l'accroissement, les 27 bivalents se rassemblent en une plaque équatoriale très semblable à celles des

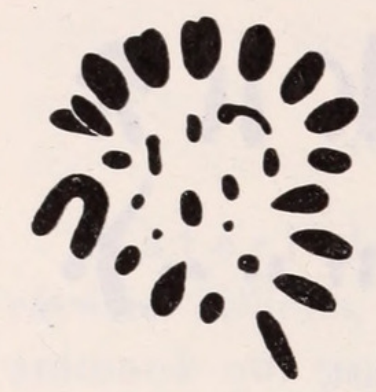

Fig. 37.

Rhinolophus hipposideros Bechst. Seconde cinèse de maturation. Hématoxyline $4000)$. deux espèces précédemment décrites; on remarque cependant, à la périphérie, un élément plus grand, en forme de 8: il correspond à la paire de grands $V$ (fig. 31-33, 35). Certaines métaphases, peu colorées, accusent l'hétérochromatie du couple $X Y$. Sa forme, sa disposition à la métaphase, son évolution ultérieure sont en tout point semblables à ce que j'ai décrit plus haut chez Rh. ferrum equinum et euryale, abstraction faite de la taille des chromosomes sexuels, un peu plus petits chez hipposideros (fig. 34 et 36).

La seconde division de maturation montre 27 dyades (fig. 37).

\section{Famille des Vespertilionidae.}

Cette famille, l'une des plus importantes de tout l'ordre, renferme la majorité des espèces indigènes. Elle est beaucoup moins homogène que celle des Rhinolophidae.

\section{Genre Myotis Kaup.}

J'ai pu capturer quatre espèces de ce genre: $M$. myotis, $M$. daubentonii, M. mystacinus, $M$. emarginatus. Chez les deux dernières, j'ai dû me borner à l'examen des divisions diploïdes, n'ayant pu capturer un seul adulte au stade convenable.

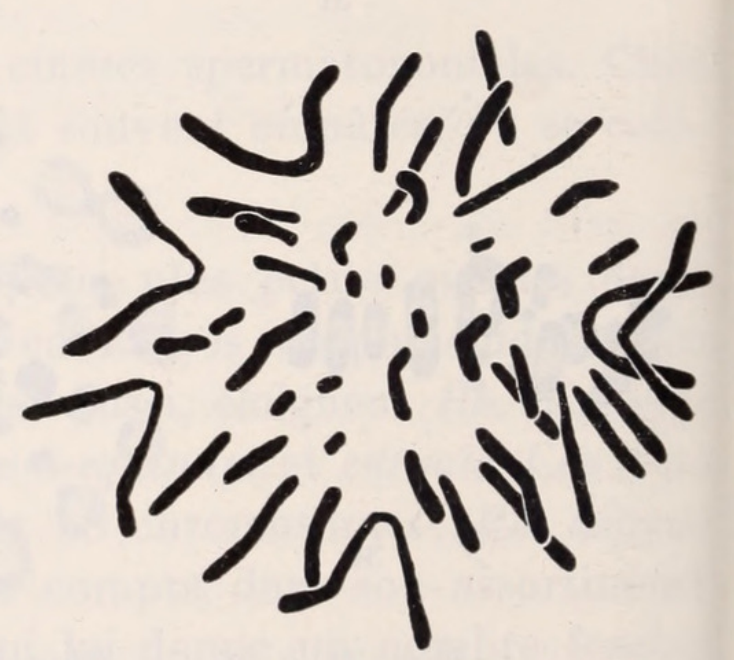

FIG. 38. - Myotis myotis Bork.

Métaphase spermatogoniale. Hématoxyline $(\times \mathbf{4 0 0 0})$. 
ne laissant rien voir de son contenu (fig. 40); mais dans d'autres cas, on peut distinguer nettement ses deux constituants $X$ et $Y$ unis par leurs extrémités (fig. 41-44) ou parfois séparés (fig. 45). Le plus grand élément - le chromosome $X$-- figure toujours une lettre $U$. Quant à son partenaire $Y$, sa position est variable: il peut être tourné vers l'intérieur (fig. 41), ou vers l'extérieur (fig. 42-45) de la concavité de l' $X$, et se trouve parfois à une certaine distance de ce dernier.

Outre l'hétérocaryosome, le noyau auxocytaire contient encore

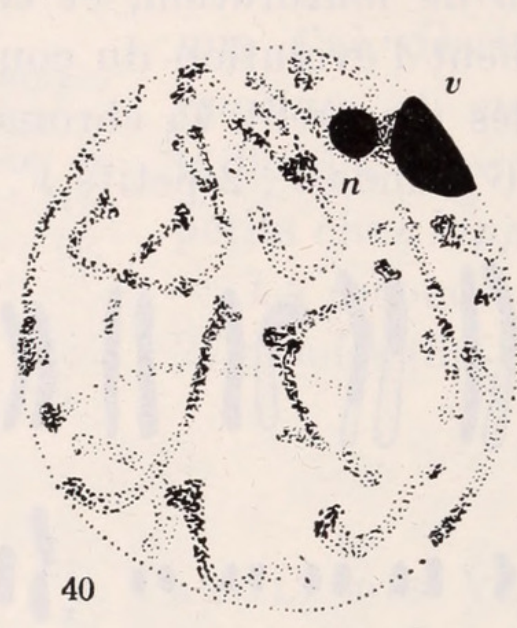

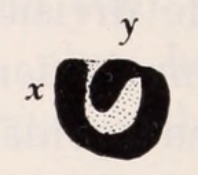

41

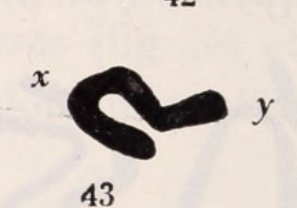

44

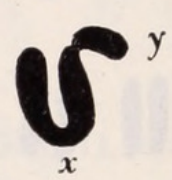

FIG. 40 à 45. - Myotis myotis Bork.

Fig. 40. - Noyau d'auxocyte au stade pachytène; $n=$ nucléole, $v=$ vésicule hétérocaryotique. FIg. 41 à 45. - Hétérochromosomes au stade pachytène. Hématoxyline $(\times 4000)$.

d'autres formations basophiles, distinctes du nucléole, et que l'on voit disparaitre lorsqu'on traite, selon la méthode de Feulgen, une préparation d'abord colorée au violet ou à l'hématoxyline.

A la diacinèse, stade dont l'observation est difficile même lorsque la cellule est bien fixée, les chromosomes sexuels ne se distinguent plus des autosomes.

La plaque équatoriale de la première division de maturation, relativement grande, se compose de 22 bivalents (fig. 46-49). On reconnaît, à la périphérie, les trois grands bivalents provenant des trois paires de grands $V$ observés dans les spermatogonies. Ils sont presque toujours disposés au bord de la plaque équatoriale, à intervalles réguliers. Les autres bivalents, formés de chromosomes acrocentriques, dessinent des anneaux, des $V$ ou des points. Quant aux hétérochromosomes, ils sont difficiles à déceler dans les méta- 
phases en vue polaire, car ils ne manifestent aucune hétérochromatie à ce stade. En revanche; certaines figures de profil (fig. 50 et 51) montrent clairement une paire hétéromorphe $X Y$, en pré-réduction. Le chromosome $X$ est métacentrique, avec des bras égaux, et son partenaire $Y$ est un bâtonnet mesurant environ le tiers de la lon-
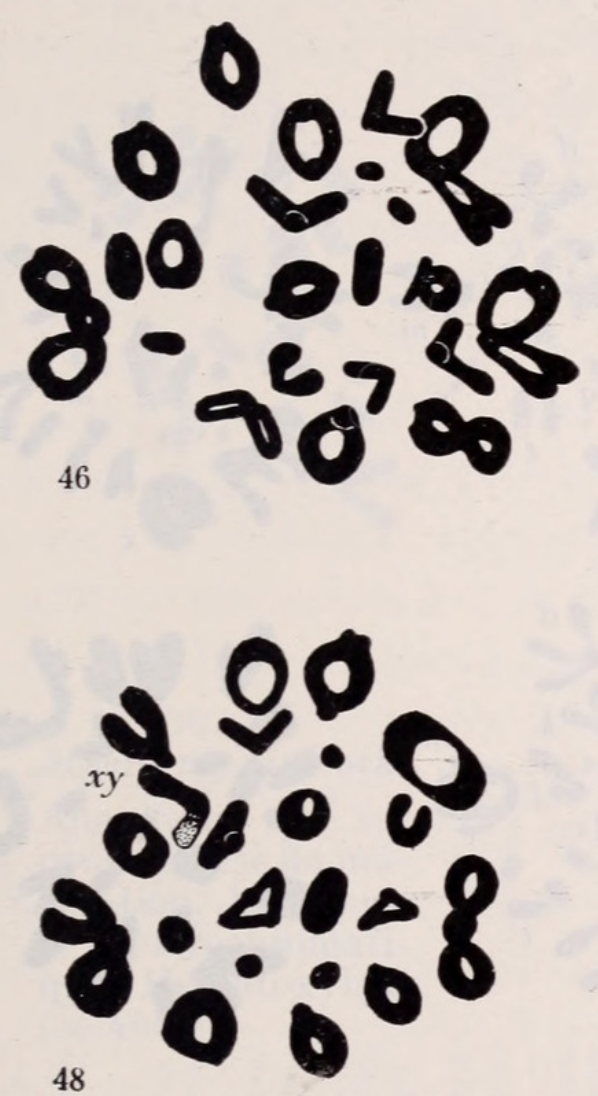
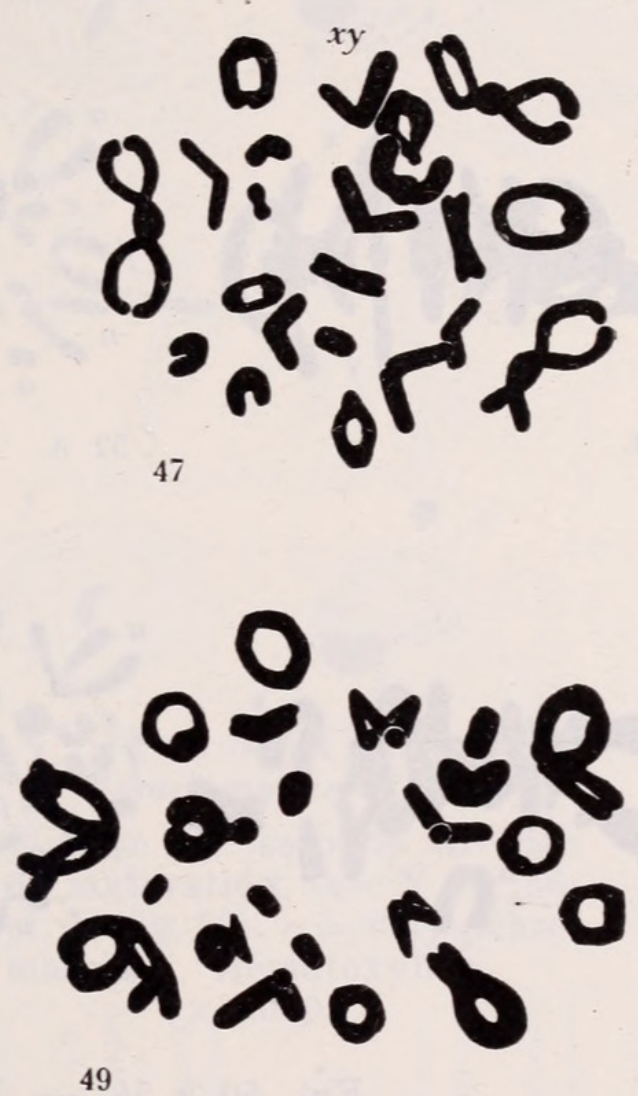

FIG. 46 à 49. - Myotis myotis Bork.

Métaphases auxocytaires.

Hématoxyline $(\times 4000)$.

gueur totale de l' $X$. A la figure 50, les deux hétérochromosomes sont encore reliés l'un à l'autre par leurs extrémités, tandis que la figure 51 marque leur séparation définitive, qui provoque un léger raccourcissement du chromosome $Y$. Sur cette dernière figure, on notera la présence, près du pôle inférieur, d'un chromosome en forme de $V$, qui n'a pas d'homologue visible dans la cellule, et ne peut donc pas être pris pour une dyade précocement séparée: il s'agit peut-être d'un univalent. Ce fait se présente d'ailleurs chez d'autres chauves-souris, les Pipistrelles notamment.

A l'anaphase, les hétérochromosomes se séparent à la même vitesse que les autosomes, dont ils ne se distinguent done pas. 
Mais, vers la fin de l'ascension polaire, ces derniers perdent leur colorabilité, tandis que les hétérochromosomes restent très foncés. Les figures $52 \mathrm{~A}$ et $\mathrm{B}$ représentent les deux pôles d'anaphase d'une première division, où l'on peut facilement discerner l' $X$, qui a pris l'aspect d'une boule relativement grosse (fig. $52 \mathrm{~A}$ ), et l' $Y$, plus petit (fig. 52 B), parmi les autosomes pâles. Cette hétérochromatie

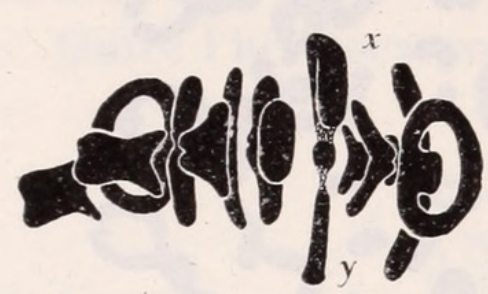

50

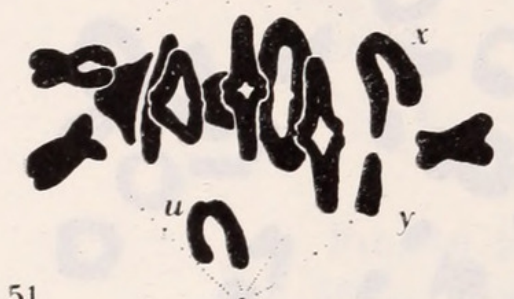

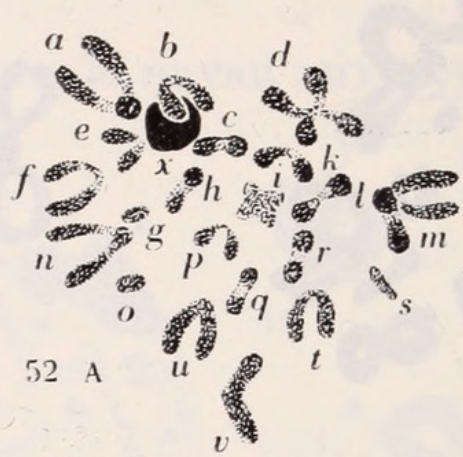

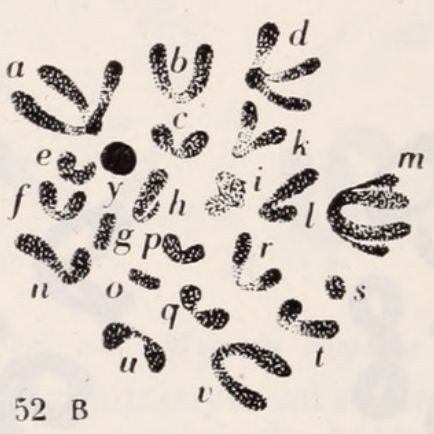

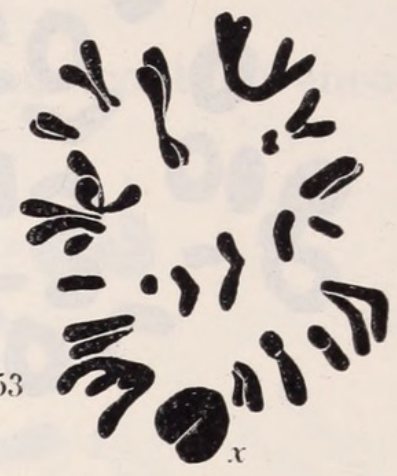

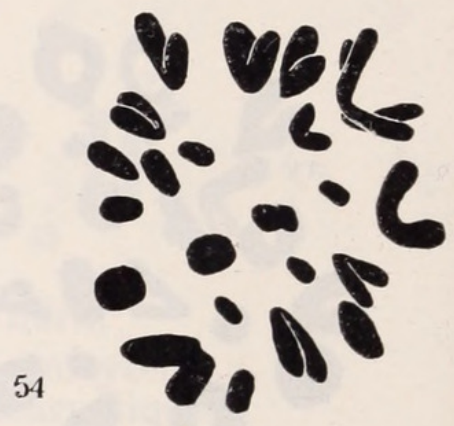

FIG. 50 à 54. - Myotis myotis Bork.

Fig. 50 et 51. Métaphases auxocytaires de profil, avec couple XY. $u=$ univalent présumé. - Fig. 52 A et B. Deux pôles d'une anaphase de première cinèse méiotique, avec $\mathrm{X}(52 \mathrm{~A})$ et $\mathrm{Y}(52 \mathrm{~B})$ hétérochromatiques. Les lettres désignent les dyades issues d'un même bivalent. Fig. 53 et 54 . Seconde cinèse de maturation. Hématoxyline $(\times 4000)$.

subsistera jusqu'à la fin de la deuxième cinèse de maturation, et, durant l'intercinèse, il est facile de reconnaître les spermatocytes porteurs de l' $X$, massif, ou de l' $Y$, plus grêle.

La seconde division de maturation est en général d'une lecture difficile. Lorsque la fixation est bonne, on compte 22 dyades (fig. 53 et 54), et l'on peut facilement distinguer les plaques équatoriales contenant la dyade $X$ de celles qui sont dotées d' $Y$. Une coloration intense, un contour plus précis et un aspect plus massif mettent en évidence l'hétérochromosome $X$ (fig. 53), aussi bien dans les coupes à l'hématoxyline que dans celles qui ont été colorées à la 
fuchsine de Feulgen. Dans les cinèses avec $Y$, le chromosome sexuel ne trahit sa présence par aucun caractère distinctif (fig. 54).

Lors de l'anaphase de la seconde division de maturation (fig. 55),

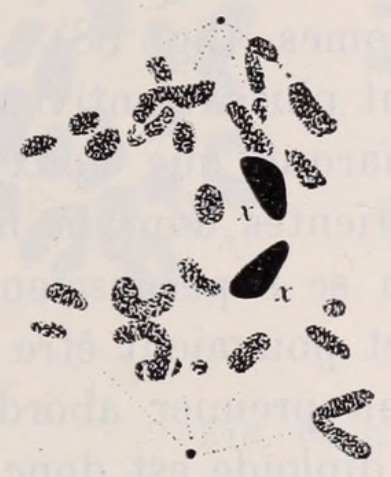

FIG. 55. Myotis myotis Bork. Anaphases de seconde cinèse de maturation. Les deux $\mathrm{X}$ hétérochromatiques. Hématoxyline $(\times 4000)$.

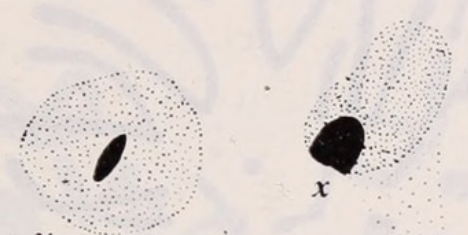

56
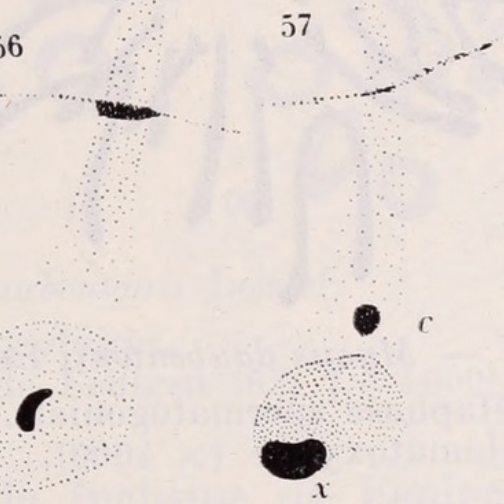

Fig. 56 et 57.

Myotis myotis Bork.

Télophase de seconde division de maturation, avec Y (fig. 56) et $\mathrm{X}$ (fig. 57). $c=$ corps chromatoïde. Hématoxyline.

$(\times 4000)$.

l'hétérochromatie d' $X$ et d' $Y$ augmente par contraste, car les monades autosomiques subissent seules une catachromatie intense. Les chromosomes sexuels restent visibles dans les spermatides, se détachant sur le fond clair du noyau dans lequel les autosomes sont complètement effacés. Ils ne s'estompent à leur tour qu'au début de la spermiogenèse.

\section{Myotis daubentonii Leisler.}

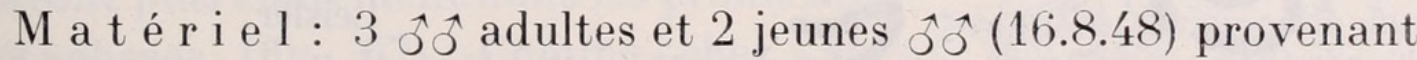
de Genève.

O b s e rvations: Le cas de cette chauve-souris est intéressant, car le nombre de chromosomes est inférieur de deux unités à celui de Myotis myotis, tandis que le nombre fondamental reste 
le même grâce à l'apparition d'une nouvelle paire de grands $V$.

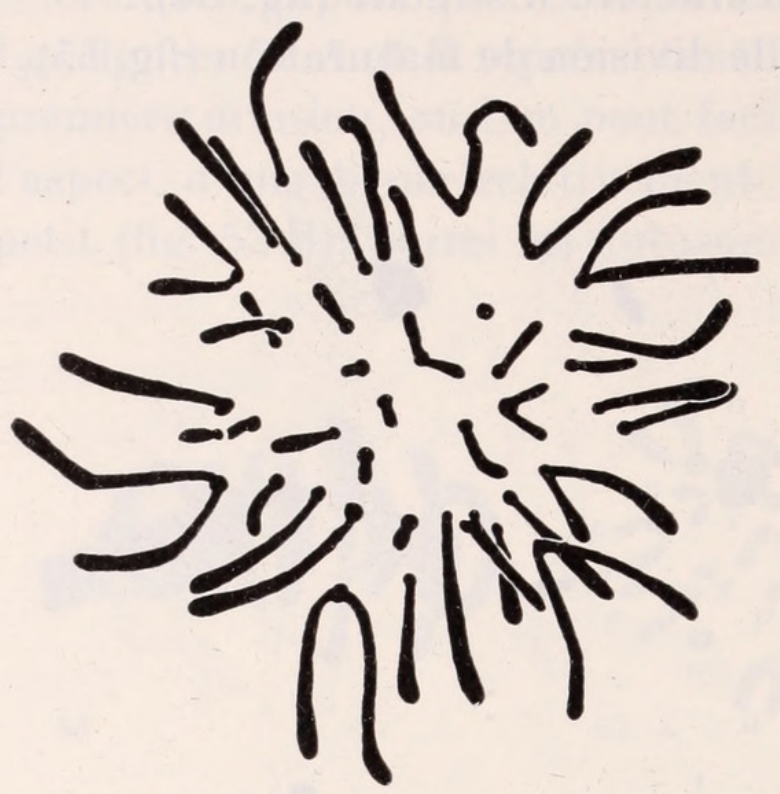

FIG. 58. - Myotis daubentonii Leisler.

Métaphase spermatogoniale. Hématoxyline $(\times \mathbf{4 0 0 0})$.

La loi de Robertson est exactement vérifiée.

Je n'ai trouvé qu'une métaphase spermatogoniale, mais elle est d'une grande clarté: j'ai tout d'abord compté 41 chromosomes (fig. 58), puis, regardant plus attentivement, j'ai remarqué que deux éléments orientés dans la même direction se superposaient en partie, et pouvaient être confondus au premier abord. Le nombre diploïde est donc égal à 42. L'assortiment (fig. 59) comprend 8 grands $V, 2$ petits
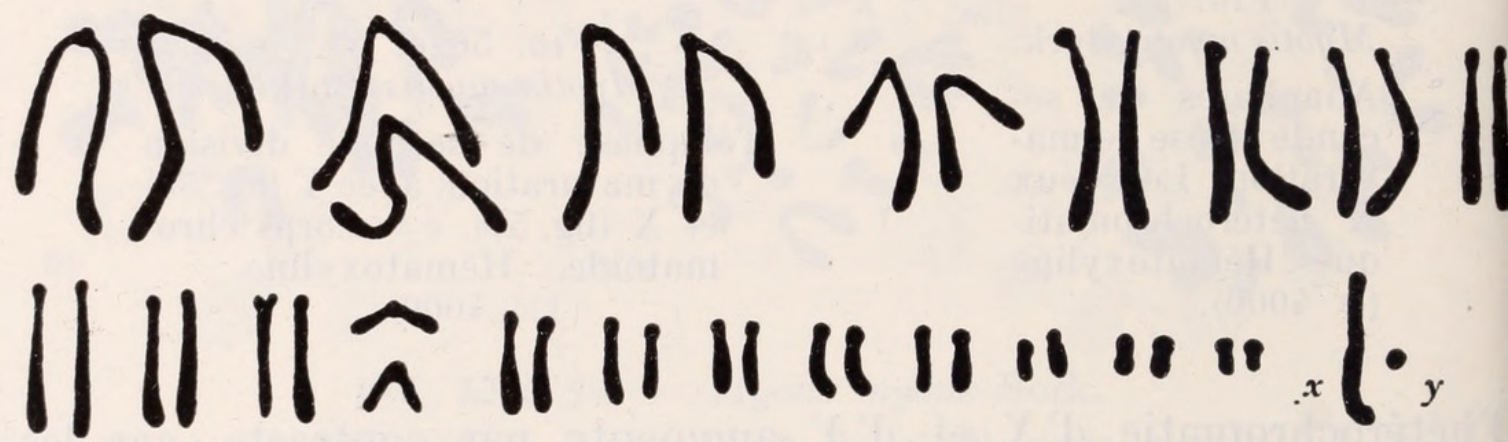

Fig. 59. - Myotis daubentonii Leisler.

Chromosomes spermatogoniaux $(\times 4000)$.

$V$ et des bâtonnets de dimensions diverses. Le nombre fonda-
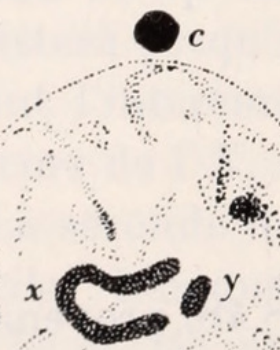

$\sum_{11} \div$

60

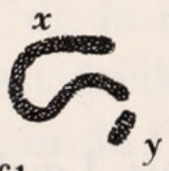

61

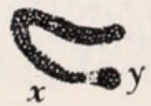

62

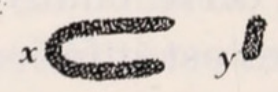

63 mental est 53, le chromosome $X$ étant métacentrique.

FIG. 60 à 63. - Myotis daubentonii Leisler.

Fig. 60. Noyau d'auxocyte au stade pachytène avec X et Y hétérochromatiques. $n=$ nucléole, $c=$ corps chromatoïde. - FIG. 61 à 63. Quelques aspects des hétérochromosomes au stade pachytène. Hématoxyline $(\times 4000)$. 
Au stade pachytène (fig. 60-63), on aperçoit dans le noyau, outre un nucléole arrondi, un long filament recourbé en $V$, en relation avec un court élément de même épaisseur et de même teinte; ces deux organites sont accolés à la face interne de la membrane nu-

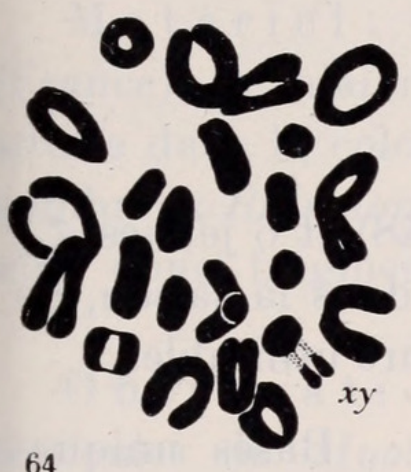

64

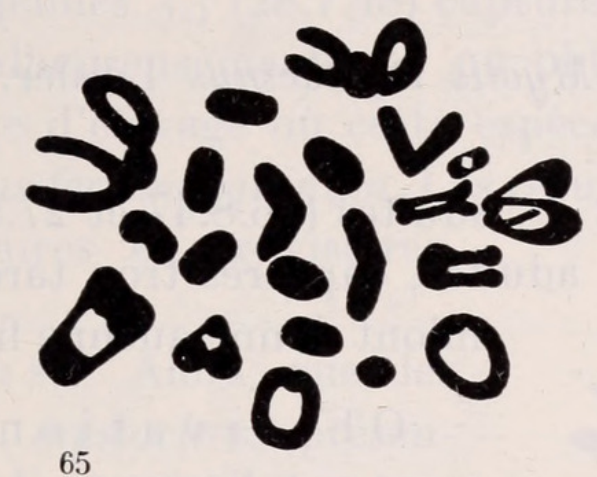

65

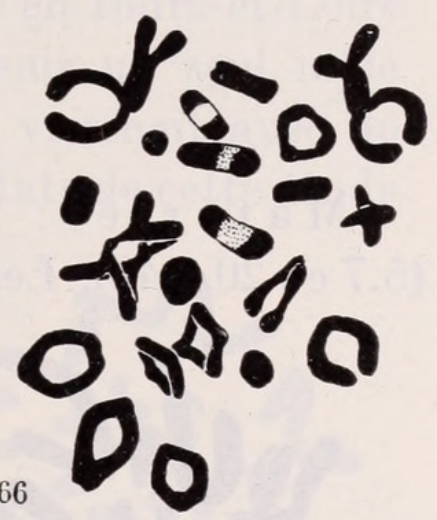

FIG. 64 à 66. - Myotis daubentonii Leisler.

Métaphases auxocytaires.

Hématoxyline $(64,66)$ et fuchsine de Feulgen $(65)(\times 4000)$.

cléaire, et se colorent aussi bien à la fuchsine de Feulgen qu'à l'hématoxyline. Dans de nombreux cas, cependant, on ne voit qu'une masse hétérochromatique opaque. Comme je l'ai déjà noté en ce qui concerne Myotis myotis, il s'agit là de l'hétérocaryosome, formé des deux chromosomes sexuels $X$ et $Y$, qui montrent chez $M$. daubentonii la même disposition que chez $M$. myotis. Il n'est pas sans intérêt de remarquer qu'à ce stade, les hétérochromosomes sont, dans de nombreux cas, complètement séparés (fig. 60 et 63 ).

La première division de maturation a le mème aspect que chez Myotis myotis (fig. 64-66), et montre 21 bivalents. Dans les cas les plus favorables, les hétérochromosomes peuvent être identifiés grâce à leur structure dyssymétrique. Ils apparaissent plus clairement dans les profils des métaphases (fig. 67). Comme chez $M$. myotis, l' $X$ est métacentrique. Il est relié

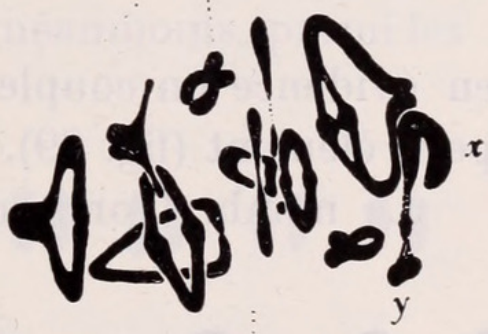

FIG. 67. - Myotis daubentonir Leisler.

Première division de maturation: anaphase de profil, avec couple XY. par l'un de ses bras à son partenaire $Y$, plus petit que chez l'espèce précédente. La préréduction semble être de règle, mais je n'ai pas examiné un nombre assez 
grand de figures pour pouvoir exclure la possibilité d'une postréduction.

Les secondes cinèses de maturation n'ont pas été analysées.

\section{Myotis mystacinus Leisler.}

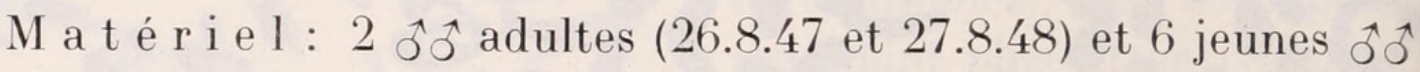
(5.7 et 20.8.48). Les adultes, capturés trop tard dans la saison, ne

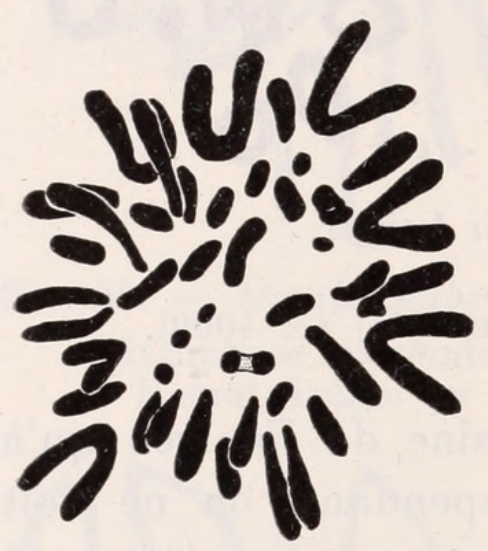

FIG. 68. - Myotis mystacinus Leisler.

Métaphase de mitose somatique (tissu interstitiel). Fuchsine de Feulgen $(\times 4000)$. m’ont donné aucune figure utilisable.

O bs e rvation s: Basés uniquement sur l'examen des cinèses spermatogoniales et somatiques, ces résultats sont évidemment moins sûrs que ceux que l'on peut obtenir par une étude complète de la spermatogenèse.

Les métaphases spermatogoniales et celles des cellules de Leydig ont 44 chromosomes (fig. 68). Comme chez M. myotis, il y a trois paires de grands $V$, et, semble$\mathrm{t}$-il, une paire de très petits $V$. Les autres éléments sont des bâtonnets de taille variable. La sériation des chromosomes est difficile: elle permet de mettre en évidence un couple $X Y$, formé d'un métacentrique et d'un très petit élément (fig. 69).

Le nombre fondamental est le même que chez Myotis myotis.

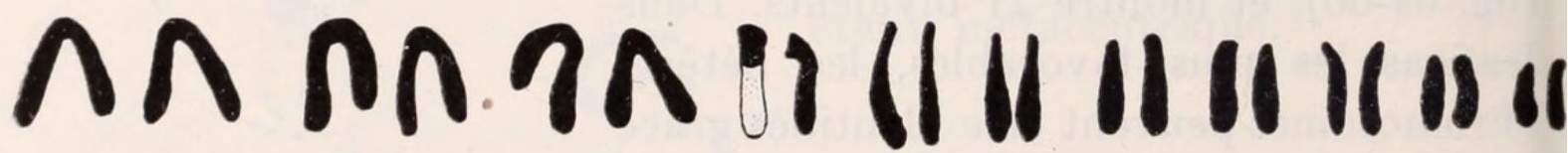

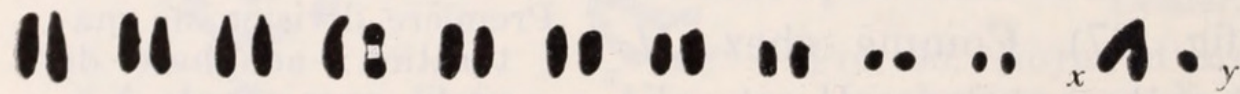

FIG. 69. - Myotis mystacinus Leisler.

Chromosomes de l'assortiment diploïde, somatique $(\times 4000)$. 
Myotis emarginatus Geoffroy.

M a té ri e l: 4 jeunes $\widehat{o} \sigma^{-1}(28.7 .48)$ capturés en Indre-et-Loire (France). Je n'ai malheureusement pas pu obtenir un seul mâle adulte dans la colonie d'élevage où cette espèce voisinait avec de nombreux Rhinolophus ferrum-equinum. Les résultats de cette étude sont done fragmentaires et provisoires.

Observations: Alors que les testicules de Myotis mystacinus présentaient dans leur tissu interstitiel de nomoreuses mitoses, M. einarginatus ne m'a courni de bonnes images que dans les ubes séminifères. (Fig. 70) Les métaohases ont un aspect très particulier: les chromosomes sont massifs, très courts et oaraissent fortement contractés. Comme e n'ai pas de matériel de comparaison, il n'est impossible de dire si cette structure orovient de la fixation, ou si elle est carac-

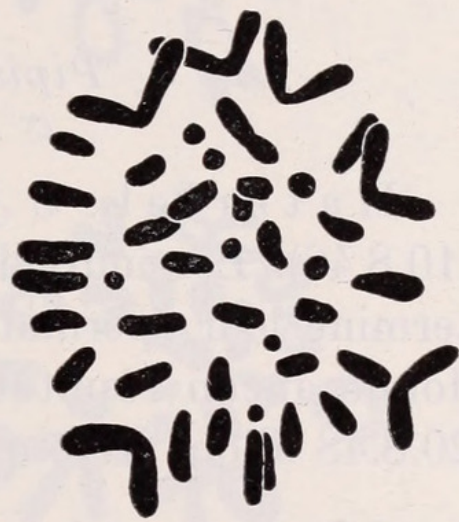

FIG. 70. - Myotis emarginatus Geoffr.

Métaphase spermatogoniale. Fuchsine de Feulgen $(\times 4000)$. éristique de l'espèce. Quoi qu'il en soit, lle facilite beaucoup la numération, mais rend très difficile l'analyse norphologique des chromosomes. On distingue néanmoins, parmi les

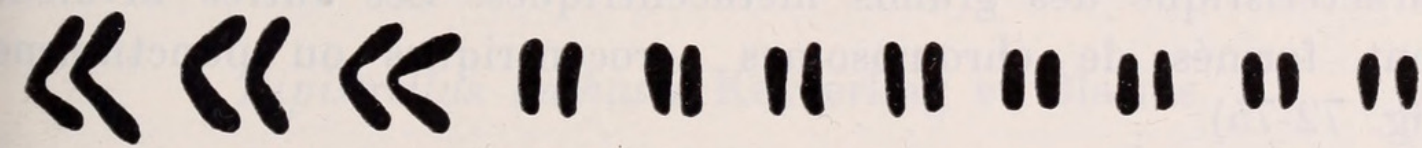

\section{$01018010800 \ldots \ldots \ldots \ldots$}

FIG. 71. - Myotis emarginatus Geoffr.

Chromosomes spermatogoniaux $(\times 4000)$.

44 chromosomes que compte cette espèce, 3 paires de grands $V$ comme chez $M$. myotis et $M$. mystacinus. Le nombre fondamental st donc approximativement 51, en admettant un $X$ métacentrique (fig. 71). 
Genre Pipistrellus Kaup.

Des quatre espèces que ce genre compte dans notre région, je n'ai pu me procurer que $P$. pipistrellus et $P$. nathusii. L'analyse montre que l'étroite parenté morphologique existant entre ces deux espèces, souvent difficiles à distinguer, n'exclut pas des différences d'ordre cytologique.

\section{Pipistrellus pipistrellus Schreber.}

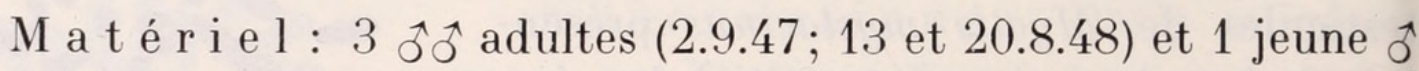
(10.8.46). Les individus capturés en septembre 1947 avaient déjà terminé leur spermatogenèse. Le jeune pris en août 1946 ne m'a donné aucun résultat. Seuls, les trois individus trouvés les 13 et 20.8.48 m'ont livré quelques figures, à vrai dire excellentes.

O b s e r vati o n s: Je n'ai pas trouvé une seule métaphase spermatogoniale claire. Les rares figures que j'ai pu voir de ce stade indiquent la présence de trois paires de grands $V$ au moins.

En revanche, j'ai relevé de nombreuses métaphases de première division de maturation, dont les chromosomes sont souvent si bien fixés que leur analyse, rendue ardue par la finesse même de la fixation et la conservation des moindres détails, est paradoxalement plus difficile que celle de figures moins parfaites. Pipistrellus pipistrellus possède 21 bivalents, dont 4 montrent la structure caractéristique des grands métacentriques. Les autres bivalents sont formés de chromosomes acrocentriques ou punctiformes (fig. 72-75).

Les métaphases de profil (fig.76) mettent en évidence le couple des hétérochromosomes; $X$ est un élément métacentrique à bras inégaux; le plus court des deux bras est en relation avec un $Y$ en forme de bâtonnet, mesurant environ $1 \mu$. L'autre bras de l' $X$ (segment différentiel ?) est en général perpendiculaire au fuseau. Je n'ai pas observé de cas certain de post-réduction, et il semble que la pré-réduction soit, ici encore, le seul mode de ségrégation des hétérochromosomes à la première division de maturation.

Les secondes divisions de maturation n'ont pas été étudiées.

Bien que les cinèses spermatogoniales n'aient pas été observées, 
on peut admettre en première approximation, d'après l'analyse des cinèses réductionnelles, qu'elles renferment 9 métacentriques,

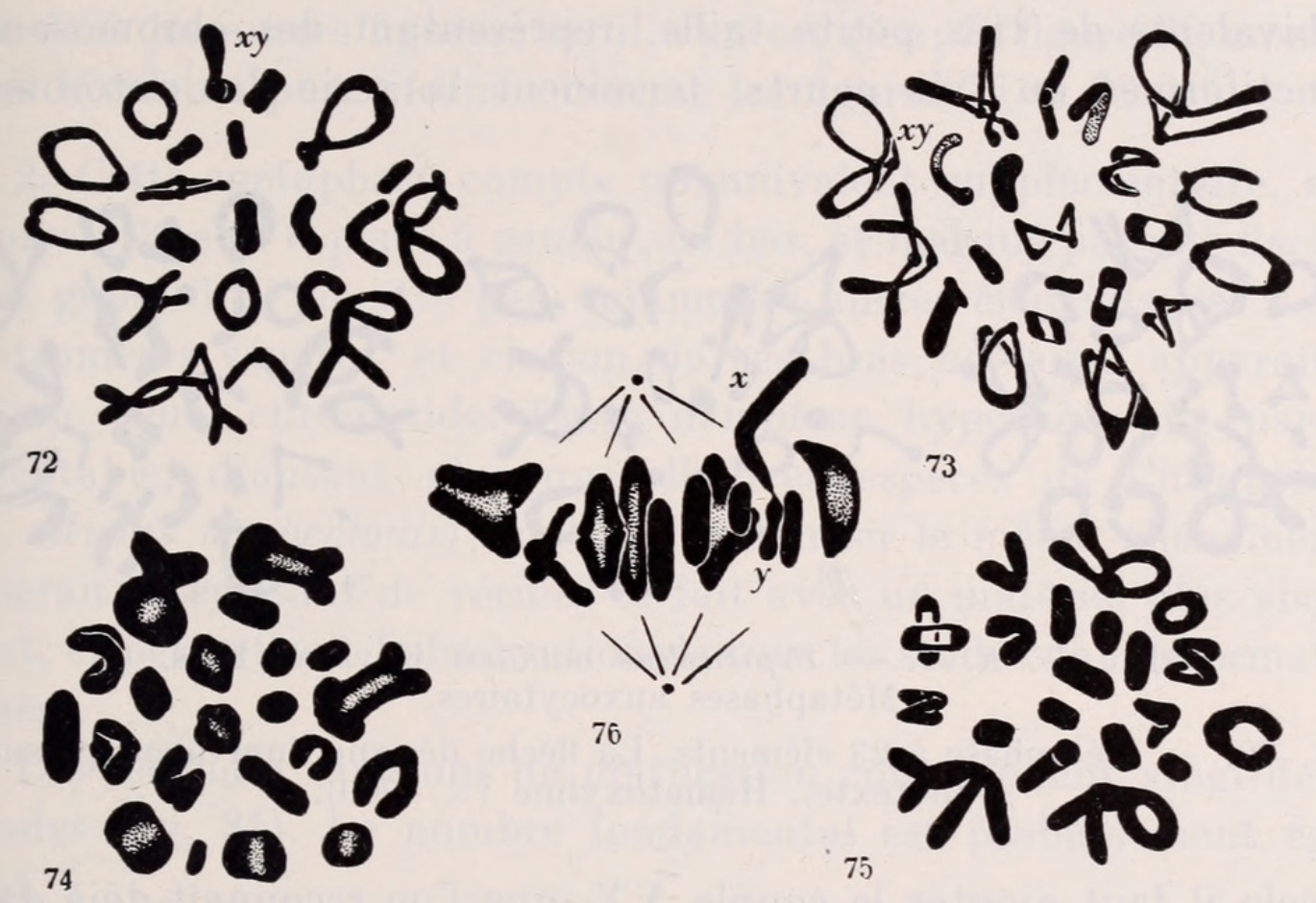

FIG. 72 à 76. - Pipistrellus pipistrellus Schr.

Métaphases auxocytaires.

FIG. 76. - Profil, avec la paire XY.

Hématoxyline, sauf la figure 74, colorée Feulgen $(\times 4000)$.

28 acrocentriques et 5 éléments punctiformes dont le mode d'attachement ne peut être décelé, mais qu'on peut considérer comme acrocentriques. Le nombre fondamental est donc 51.

Pipistrellus nathusii Keyserling et Blasius.

M a t é ri e l : 2 ડ̧ô adultes $(5.9 .47 ; 13.8 .48)$ dont le second seul a été utilisé.

O b s e r v a t i o n s: Pipistrellus nathusii a deux chromosomes de plus que $P$. pipistrellus. Comme chez cette dernière espèce, je n'ai $\mathrm{pu}$ observer que les divisions réductionnelles, mais leur analyse ne laisse aucun doute à ce sujet: les métaphases de première division de maturation (fig. 77-79) comprennent 22 bivalents parmi lesquels on peut dénombrer 3 éléments de structure métacentrique, identiques aux grands bivalents de l'espèce précédente, et de 
taille comparable. Il y a en outre 15 bivalents formés de chromosomes acrocentriques, qui dessinent soit des anneaux, soit des $V$ couchés dans le plan équatorial ou dressés verticalement. Enfin, 3 bivalents de très petite taille, représentant des chromosomes punctiformes ou très courts, terminent la série des autosomes.
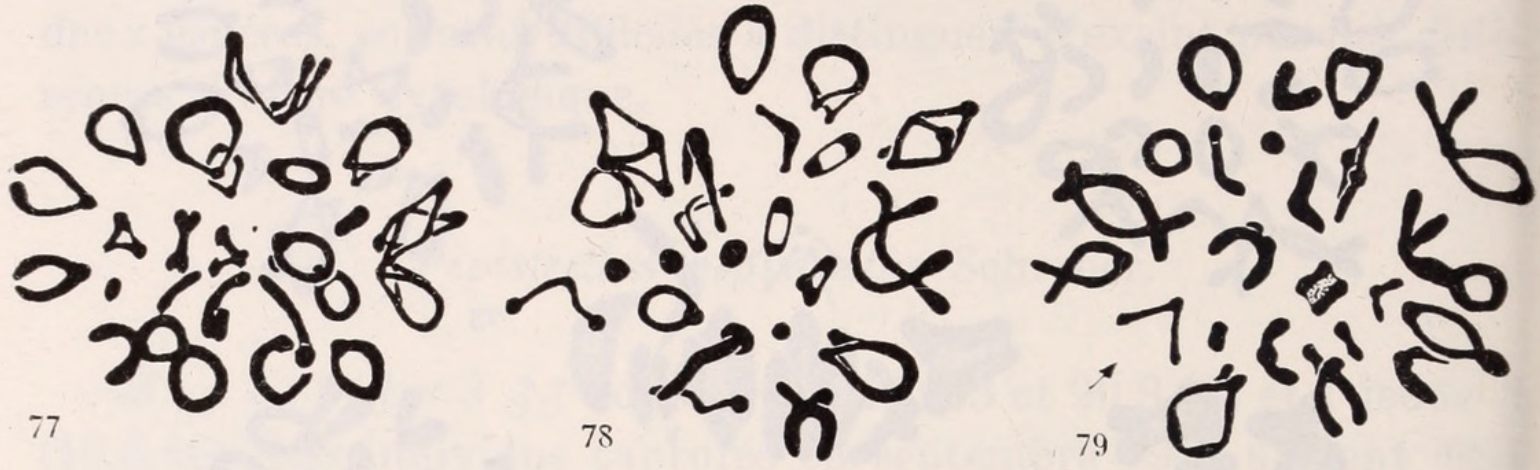

FIG. 77 à 79. - Pipistrellus nathusii Keys. et Blas.

Métaphases auxocytaires.

FIG. 79. - Métaphase à 23 éléments. La flèche désigne l'univalent présumé (voir texte). Hématoxyline $(\times 4000)$.

A cela il faut ajouter le couple $X Y$, que l'on reconnait déjà dans les figures en vue polaire, mais qui apparaît mieux de profil (fig. 80). Il est en tout point semblable à celui de $P$. pipistrellus, et

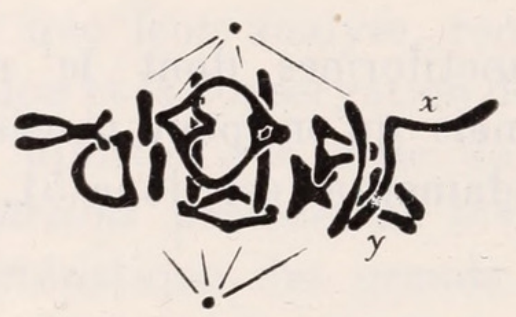

FIG. 80.

Pipistrellus nathusii Keys. et Blas.

Métaphase auxocytaire, vue de profil, avec paire XY. Hématoxyline. $(\times 4000$.

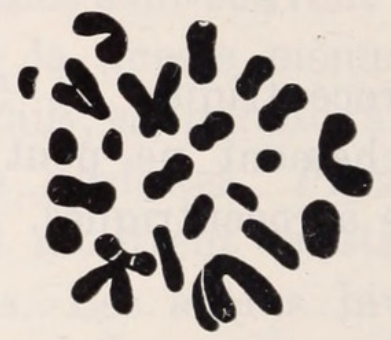

FIG. 81 .

Pipistrellus nathusii Keys. et Blas.

Seconde cinèse de maturation.

Hématoxyline. $(\times 4000$. $)$

se compose d'un $X$ métacentrique et d'un élément très petit, l' $Y$. Je n'ai observé que des figures de pré-réduction.

La figure 79 reproduit une métaphase auxocytaire où l'on peut compter 23 chromosomes. D'une fixation excellente, bien étalée, cette figure ne laisse aucun doute quant à l'exactitude de la numération. Deux explications se présentent à l'esprit: 
1. L'un des éléments punctiformes est un nucléole ou un corps chromatoïde qui se trouve par hasard dans le plan équatorial. Dans ce cas, le nombre des petits éléments devrait être plus grand que dans les autres figures; or, il ñ'en est rien; la figure 79 montre trois bivalents punctiformes comme les figures 77 et 78 .

2. Cette métaphase compte un univalent supplémentaire, qui pourrait être le $V$ placé à gauche, en bas, et marqué par une flèche. Très grêle, il ne montre pas, comme les autres éléments, ses deux centromères séparés, et chacun de ses bras ne laisse apparaître qu'une seule chromatide. Cette deuxième hypothèse me paraît acceptable, d'autant plus que d'autres espèces de Chiroptères (cf. Myotis daubentonii) semblent présenter le même phénomène. Il serait intéressant de vérifier ce fait avec un matériel plus abondant, et permettant l'observation de tous les stades de la spermatogenèse.

Les secondes divisions de maturation comprennent vingt-deux dyades (fig. 81). Le nombre fondamental est probablement égal à 51 .

\section{Miniopterus schreibersii Natterer.}

M a téri e l: Tous les mâles étudiés proviennent de la grotte de chemin de fer, à Chambrelien-sur-Neuchâtel: 11 adultes et 1 jeune ont été fixés en avril, août et novembre. Les divisions spermatogoniales ont été observées chez les sujets fixés au début de juillet, et les divisions réductionnelles en août. Chose curieuse, les individus fixés à la même date, mais à une année d'intervalle, ont montré de très grandes différences quant à l'état du testicule. En 1946 la spermatogenèse s'est déroulée avec près d'un mois de retard par rapport à 1947, année beaucoup plus chaude et moins pluvieuse.

O b s e r v a ti o n s: La seule division

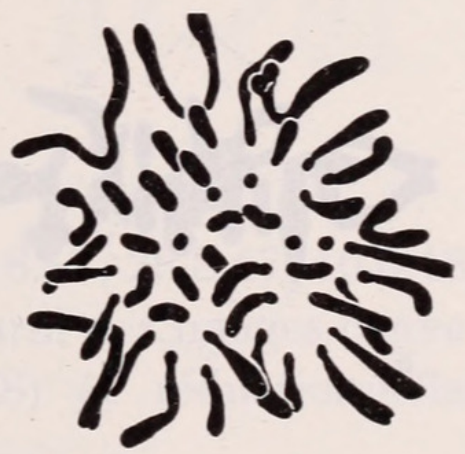

FIG. 82.

Miniopterus schreibersii Natt.

Métaphase spermatogoniale. Trichromique de Flemming. $(\times$ 4000. $)$ spermatogoniale claire que j'ai pu dessiner (fig. 82) est de petite taille, mais on y compte sans difficulté 46 
chromosomes. Les éléments acrocentriques sont en majorité, mais on remarque cependant deux paires de grands $V$ et deux

$\langle(\langle<\rangle\|\|\|\|\|\|\|\| x$

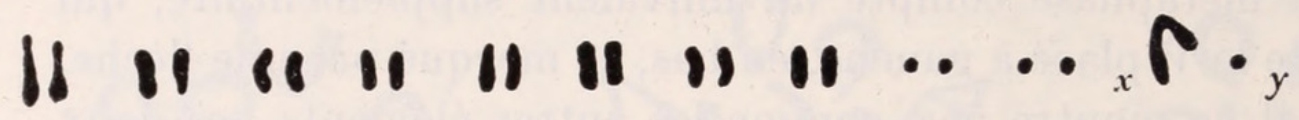

FIG. 83.

Miniopterus schreibersii Natt.

Chromosomes spermatogoniaux. $(\times 4000$. $)$

paires de chromosomes punctiformes. L'appariement en un caryogramme (fig. 83) permet de déceler une paire hétéromorphe $X Y$, formée d'un $X$ métacentrique de taille moyenne, et d'un $Y$
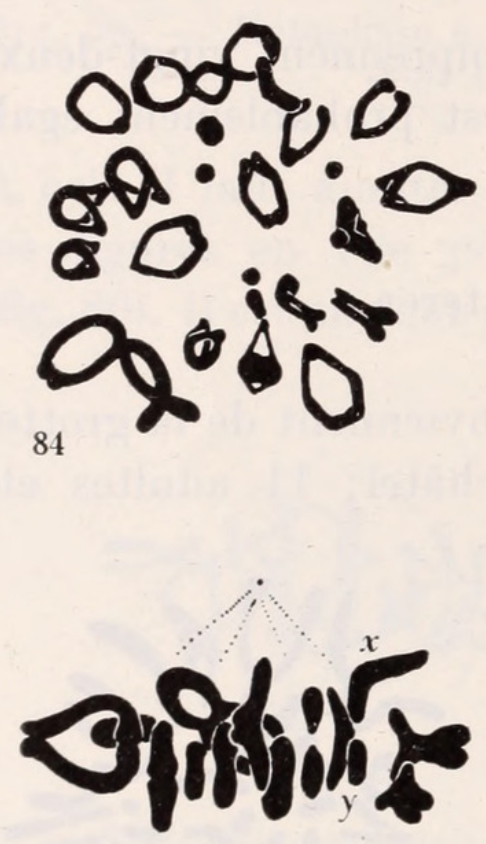

87
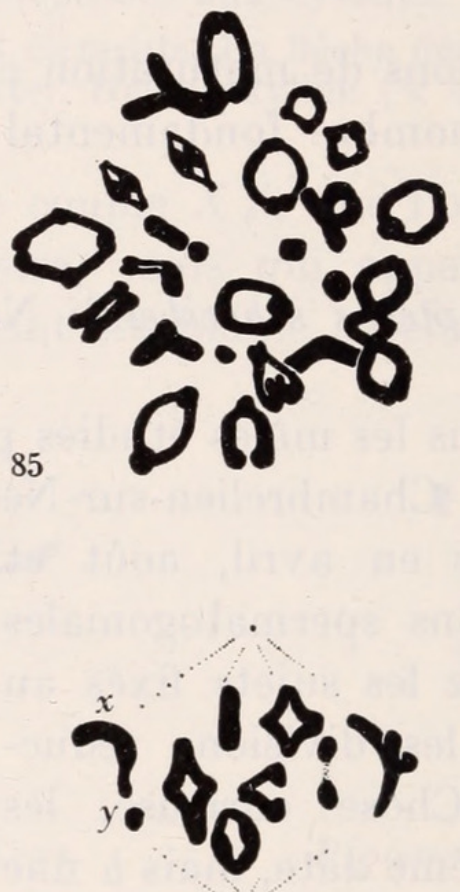

88

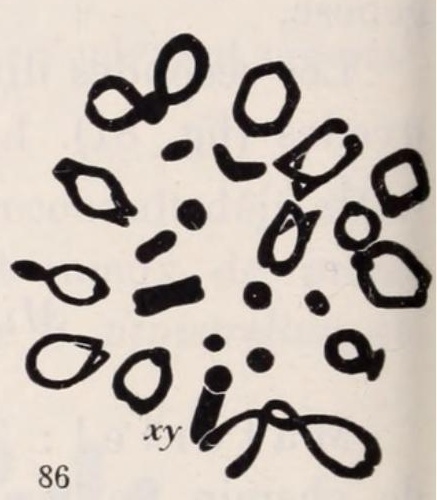

y

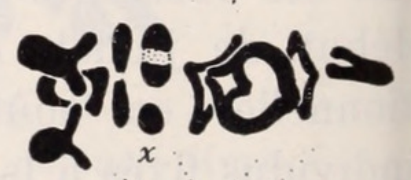

89

FIG. 84 à 89.

Miniopterus schreibersii Natt.

Métaphases auxocytaires, en vue polaire (84-86), et de profil (87-89). Hématoxyline. $(\times 4000$.

punctiforme. Tenant compte de ces données, on peut considérer le nombre fondamental comme étant égal à 51 chez cette espèce.

On compte 23 bivalents dans les métaphases de première cinèse, parmi lesquels les grands éléments métacentriques attirent 
l'attention (fig. 84-86). Formés en général de deux ou trois boucles, ils sont toujours placés vers le bord de la plaque équatoriale. Les autres bivalents dessinent des anneaux simples, des $V$ ou des points. L'un des éléments possède une dyssymétrie frappante: il se compose d'une branche horizontale toujours tournée vers l'extérieur de la plaque équatoriale, et d'une branche verticale, surmontée d'un petit chromosome. On a reconnu là le complexe $X Y$ en pré-réduction, dont l'aspect ne diffère pas essentiellement de celui qu'il a chez Myotis et Pipistrellus. Quelques cinèses de profil (fig. 87-89) le montrent très nettement: les deux bras de l'X apparaissent légèrement inégaux, le bras apparié étant, ici encore, le plus court. La pré-réduction semble constante.

Les deux grands éléments métacentriques sont facilement reconnaissables dans la plaque équatoriale des deuxièmes divisions de maturation (fig. 90), où ils montrent en général leurs deux chromatides séparées, dessinant un double $V$ typique. Les dyades acrocentriques ont souvent la forme d'un $V$ simple, formé par l'écartement de leurs chromatides, réunies au niveau du centromère seulement. Il y a vingt-trois dyades en tout, et les hétérochromosomes ne sont pas identifiés.

\section{Plecotus auritus L.}

M a t ér i e l : 4 ô ô adultes $(30.12 .46 ; 20.8 .47 ; 28.8 .47 ; 10.6 .48)$ et 1 jeune 3 . Deux de ces animaux m'ont fourni des images convenables de cinèses spermatogoniales (10.6.48) et réductionnelles (28.8.47).

$\mathrm{O}$ b s e r v a ti o n s: L'aspect des tubes séminifères diffère de celui qu'il présente chez les autres chauves-souris, et montre beaucoup d'analogie avec Barbastella: dimensions semblables des cellules, répartition identique des différents stades, importance relative du tissu interstitiel, bien développé chez les deux espèces.

Mais l'analyse des chromosomes témoigne encore mieux de la Rev. Suisse de Zool., T. 56, 1949. 
parenté étroite existant entre Plecotus auritus et Barbastella barbastellus.

Les divisions spermatogoniales (fig. 91 et 92) ont 32 chromosomes. Leur appariement en un caryogramme est extrêmement

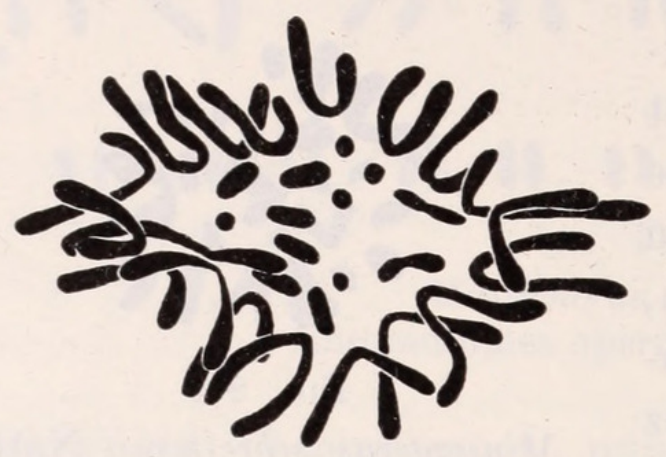

91

92

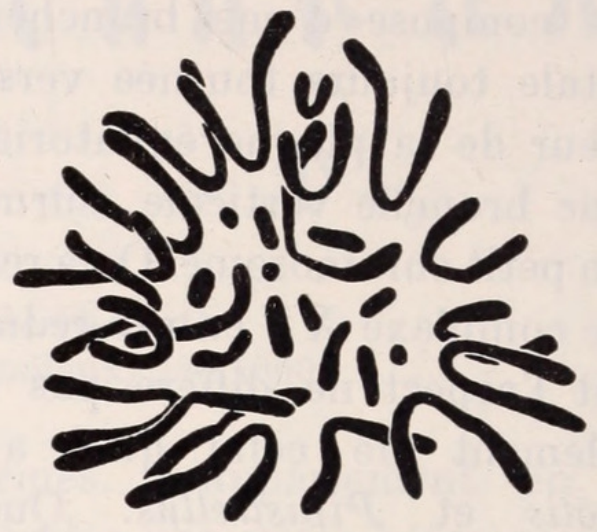

FIg. 91 et 92 .

Plecotus auritus L.

Métaphases spermatogoniales.

Fuchsine de Feulgen (91), hématoxyline (92). ( × 4000.)

facile, car les diverses catégories de chromosomes, grands $V$, bâtonnets, points, sont ici nettement distinctes, sans éléments intermédiaires (fig. 93).

Il y a neuf paires de grands $V$, une paire de petits $V$, trois paires d'acrocentriques, deux paires de chromosomes punctiformes,

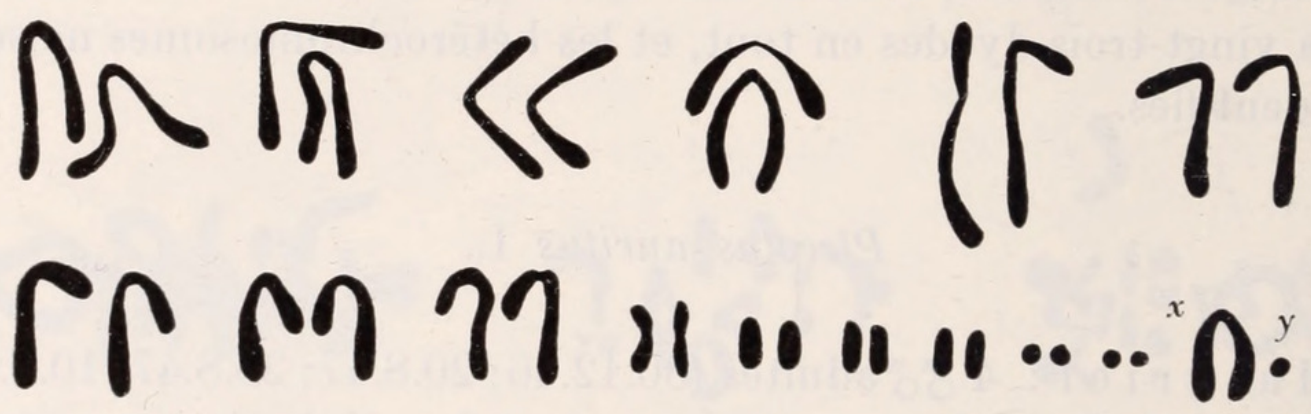

FIG. 93.

Plecotus auritus L.

Chromosomes spermatogoniaux. $(\times 4000$. $)$

et un couple $X Y$ formé, comme chez toutes les chauves-souris étudiées jusqu'ici, d'un $X$ métacentrique et d'un $Y$ de petite taille. La plupart des $V$ sont à la périphérie, et les éléments plus petits se rangent au centre.

A la métaphase de la première division de maturation (fig. 94-96) les bivalents formés par les chromosomes en $V$ sont presque tous 
complètement dissociés: seules les extrémités des chromatides sont encore réunies, les chiasmas s'étant déjà terminalisés, et l'ensemble

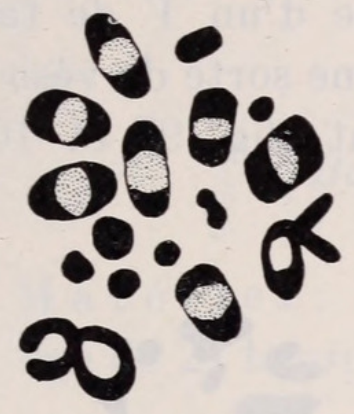

94

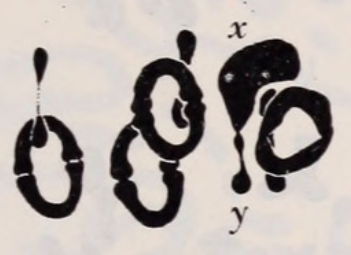

97

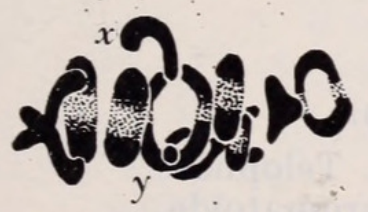

99

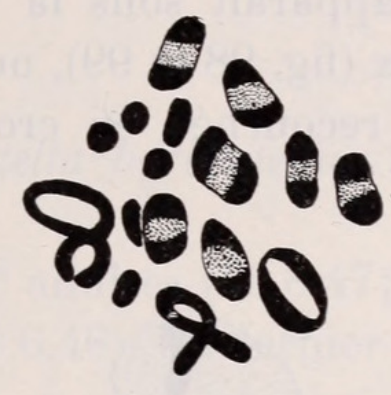

95

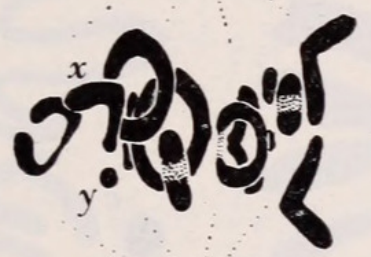

98

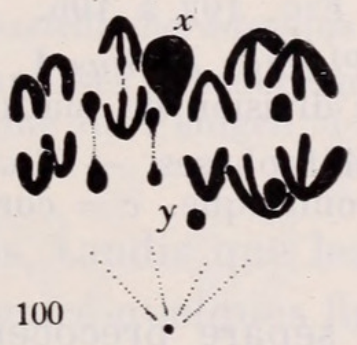

FIG. 94 à 101.

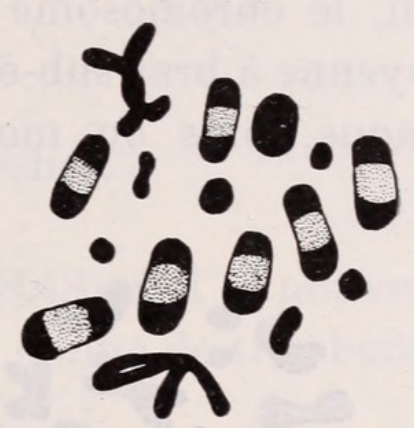

96
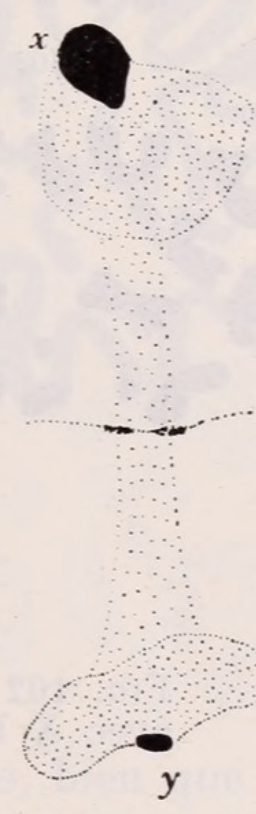

101

Plecotus auritus L.

FIG. 94 à 96, Métaphases auxocytaires en vue polaire. - Fig. 97 à 99. Métaphases auxocytaires vues de profil, avec paire XY. - FIG. 100. Anaphase. - Fig. 101. Télophase de première division de maturation. $\mathrm{X}$ et $\mathrm{Y}$ hétérochromatiques. Hématoxyline. $(\times 4000$.)

prend l'aspect d'un anneau vertical, bien visible dans les figures de profil (fig. 97-99), mais difficile à représenter en vue polaire. Cependant, deux bivalents font en général exception, formant alors une ou deux boucles dans le plan équatorial. Les autres éléments sont beaucoup plus petits, et échappent à l'analyse. Le couple $X Y$ est aisément identifié: en tournant lentement la vis micrométrique, on voit successivement une masse volumineuse, puis une tige verti- 
cale, au bout de laquelle apparaît un petit point (fig. 96). Les figures de profil (fig. 97-100) confirment cette structure. Suivant la fixation, le chromosome $X$ apparaît sous la forme d'un $V$ de taille moyenne à bras sub-égaux (fig. 98 et 99), ou d'une sorte de vésicule opaque, plus ou moins recourbée en croissant (fig. 97 et 100).

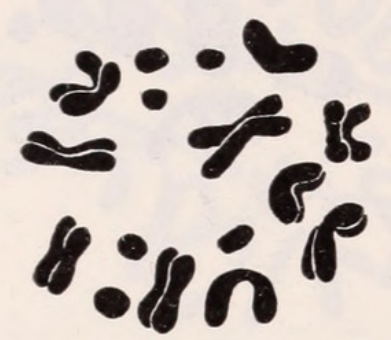

102

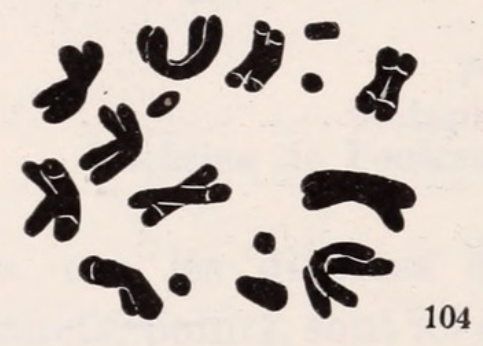

104
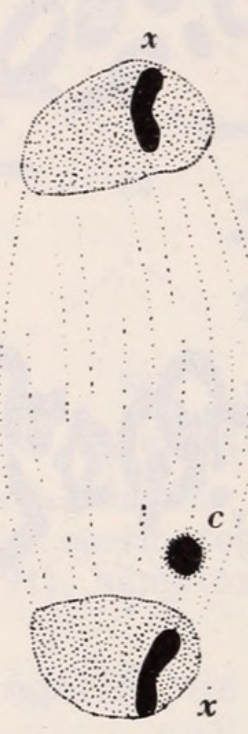

106

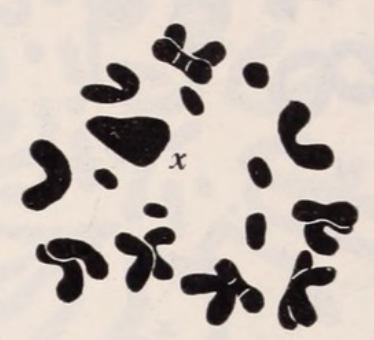

103

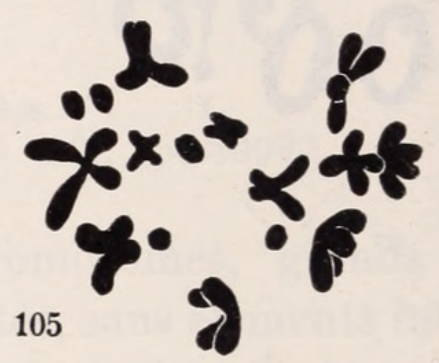

FIG. 102 à 106.

Plecotus auritus L.

Seconde division de maturation.

FIg. 102 à 105. Métaphases. - Fig. 106. Télophase avec $\mathrm{X}$ hétérochromatique. $c=$ corps chromatoïde.

L' $Y$ est très petit, et se sépare précocement de son partenaire.

A la télophase de la première division de maturation, les chromosomes sexuels sont hétérochromatiques, et apparaissent en noir intense sur le fond pâle des deux noyaux issus d'un même auxocyte. Comme chez Myotis, leur différence de taille permet de reconnaittre facilement les noyaux porteurs d' $X$ et ceux qui contiennent $Y$. Il y a toujours pré-réduction.

A la seconde division de maturation, on peut compter 16 dyades, parmi lesquelles 9 ou 10, selon les cellules, ont la structure caractéristique des éléments métacentriques de grande taille (fig. 102-105). L'hétérochromatie reparaît à l'anaphase de la seconde division de maturation, et il est en général facile de distinguer, dans les noyaux télophasiques, les chromosomes $X$ ou $Y$ (fig. 106) de structures très différentes. L'examen des couples de tels noyaux, 
où l'on trouve toujours, de part et d'autre, soit $X$, soit $Y$, est une preuve supplémentaire de la pré-réduction des hétérochromosomes.

Barbastella barbastellus Schreber.

M a t é ri e l: 5 ô ô adultes $(26.8 .47 ; 20.8 .48)$ et 1 ô nouveauné, âgé de 24 heures (23.6.48). Ce dernier m'a donné de très bonnes

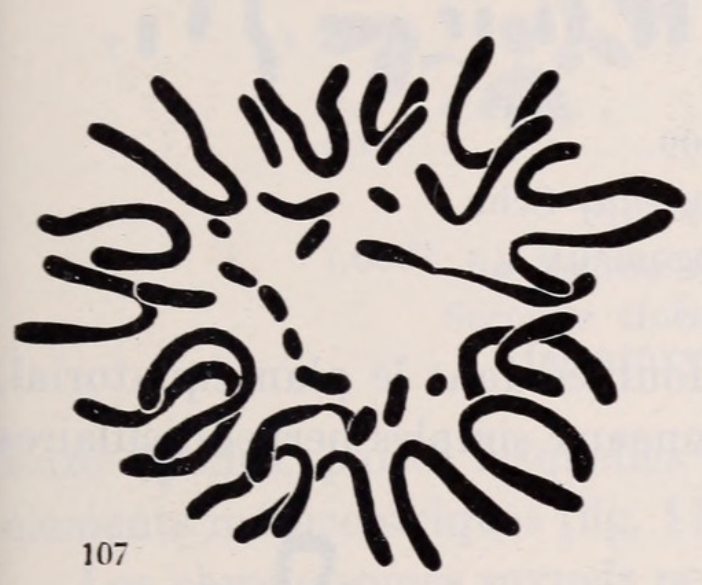

108

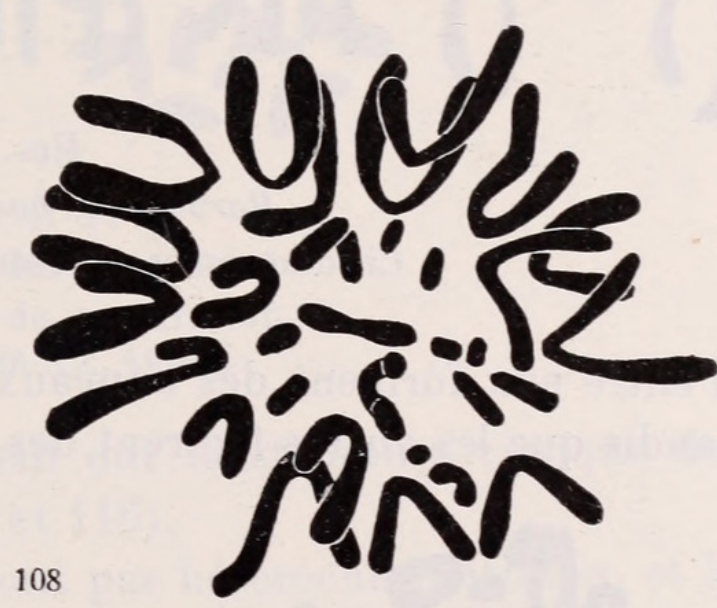

Fig. 107 et 108.

Barbastella barbastellus Schr.

Métaphases spermatogoniales.

Fuchsine de Feulgen. $(\times 4000$.

mitoses spermatogoniales, tandis que les adultes, bien que médiocrement fixés, m'ont procuré quelques figures de cinèses réductionnelles satisfaisantes.

O b s e r v a t i o n s : La garniture chromosomique, telle qu'on peut l'examiner dans les divisions spermatogoniales (fig. 107 et 108) montre une telle analogie avec celle de Plecotus auritus qu'on pourrait facilement confondre les figures provenant des deux espèces. Les 32 chromosomes sont disposés de la même façon, etles caryogrammes des deux espèces sont presque superposables (fig. 109). On retrouve neuf paires de grands $V$, une paire de petits $V$, quatre paires de chromosomes acrocentriques, une paire de chromosomes punctiformes, et un couple $X Y$, formé d'un $X$ métacentrique et d'un petit $Y$ punctiforme.

Le nombre haploïde, déterminé à la première division de maturation, confirme les données acquises par l'étude des cinèses sper- 
matogoniales (fig. 110-113). Les seize bivalents ont avec ceux de Plecotus auritus une ressemblance très frappante: trois ou quatre

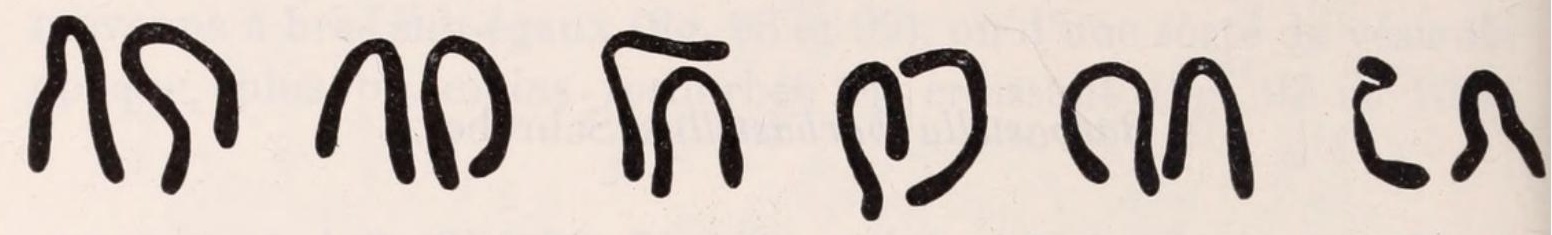

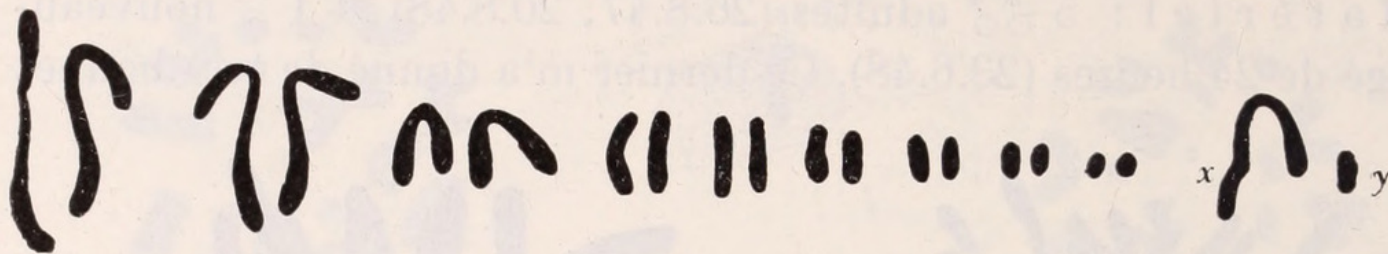

FIG. 109.

Barbastella barbastellus Schr.

Chromosomes spermatogoniaux. $(\times 4000$.

d'entre eux forment des anneaux doubles dans le plan équatorial, tandis que les autres figurent des anneaux simples perpendiculaires

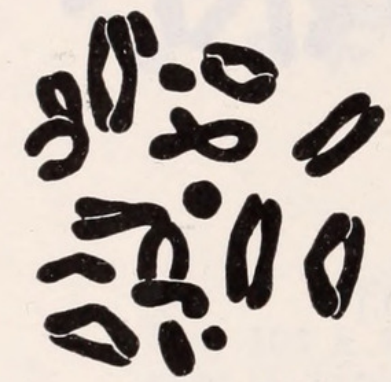

110

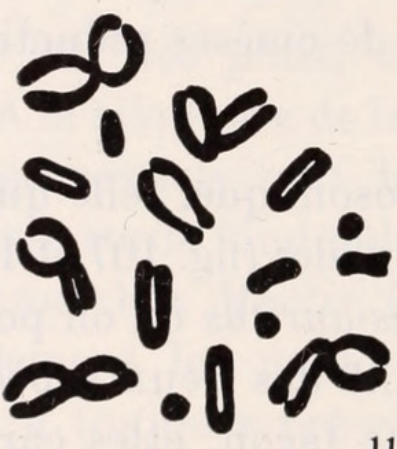

112

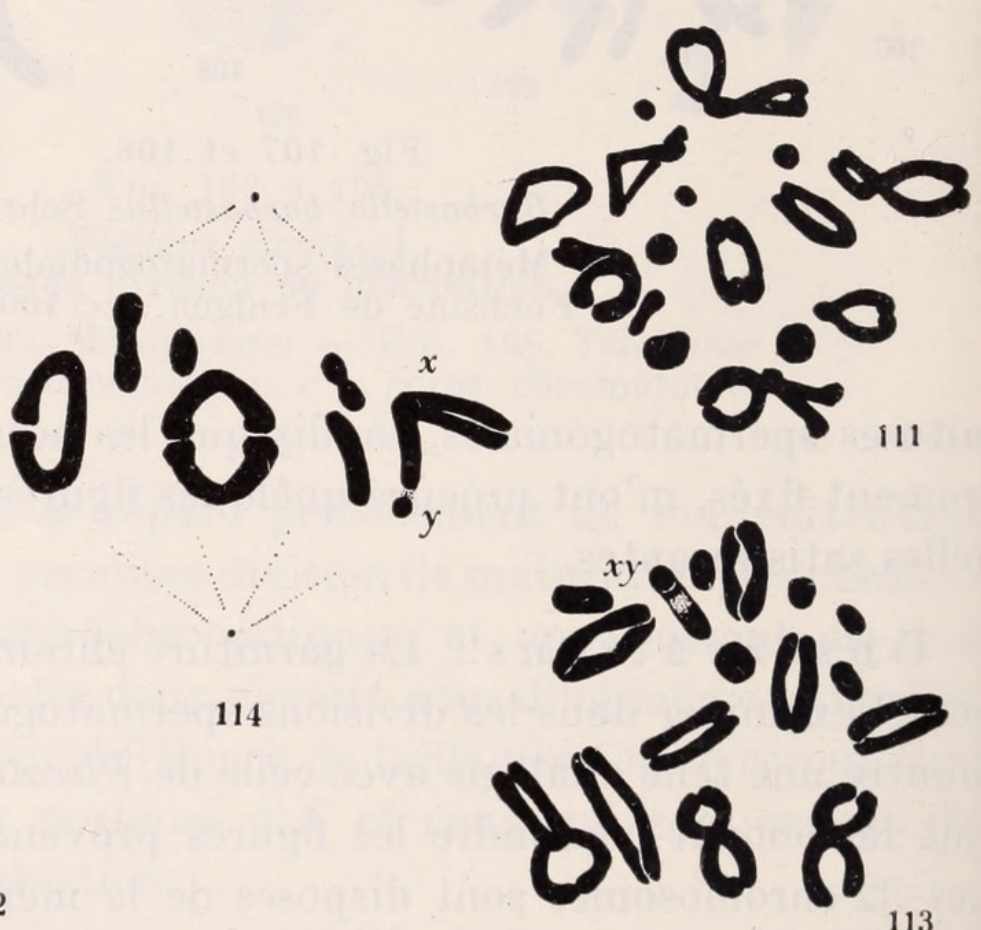

113

FIG. 110 à 114.

Barbastella barbastellus Schr.

Métaphases auxocytaires en vue polaire (110-113) et de profil (114). Hématoxyline. $(\times 4000$.

au plan équatorial, leurs chiasmas étant complètement terminalisés. Les plus petits éléments sont arrondis et condensés, et leur analyse 
s'avère difficile. Quant au complexe $X Y$, il est identique à celui de Plecotus auritus. Reconnaissable dans les métaphases auxocytaires en vue polaire, il montre bien sa structure hétérogène lorsqu'il apparaît de profil (fig. 114).

Les secondes divisions de maturation permettent de dénombrer
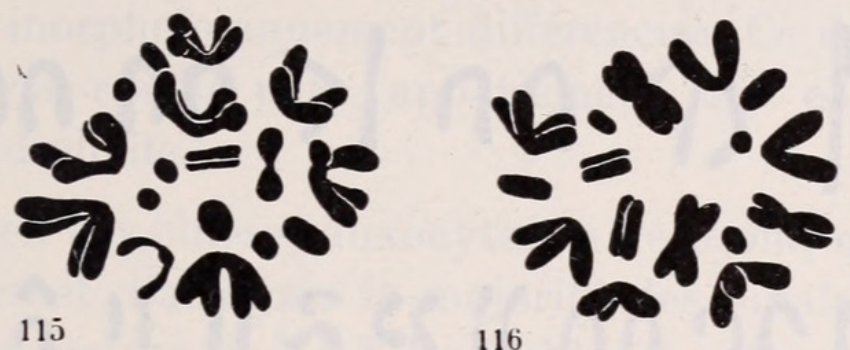

Fig. 115 et 116.

Barbastella barbastellus Schr.

Seconde cinèse de maturation.

Hématoxyline $(\times \mathbf{4} 000$. $)$

seize dyades, parmi lesquelles neuf ont la structure typique des éléments métacentriques (fig. 115 et 116).

Les chromosomes sexuels ne sont pas hétérochromatiques, et la pré-réduction semble constante.

\section{Famille des Nycteridae.}

\section{Genre Nycteris Geoffroy.}

M a t é r i e l : 1 ô adulte, fixé en juin, à Tibati (Cameroun), et dont la détermination spécifique n'a pas encore été faite.

O b s e r vations: Tous les stades de la spermatogenèse sont présents. Les cinèses réductionnelles, très mal fixées, n'ont pas donné de renseignements utiles. En revanche, quelques mitoses spermatogoniales permettent une analyse qui, si elle ne donne pas une certitude absolue, bien chimérique en

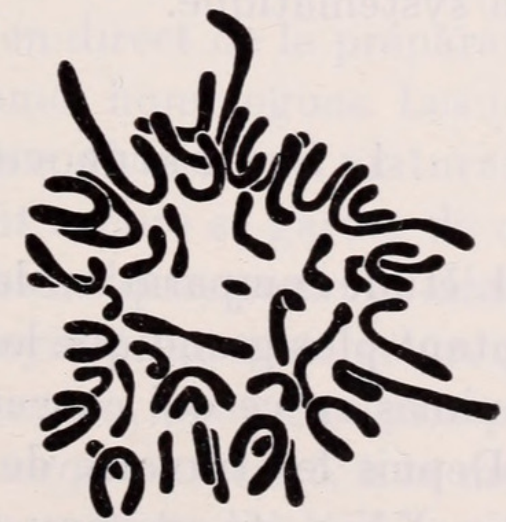

FIG. 117: Nycteris sp.

Métaphase spermatogoniale. Hématoxyline. $(\times 4000$. 
l'absence de métaphases auxocytaires, n'en a pas moins un réel intérêt documentaire, puisque aucun Nycteridae n'a été jusqu'à ce jour l'objet d'une étude cytologique (fig. 117 et 118).

Le nombre diploïde de Nycteris sp. est de 42 chromosomes, dont la plupart sont métacentriques. Un très petit élément puncti-

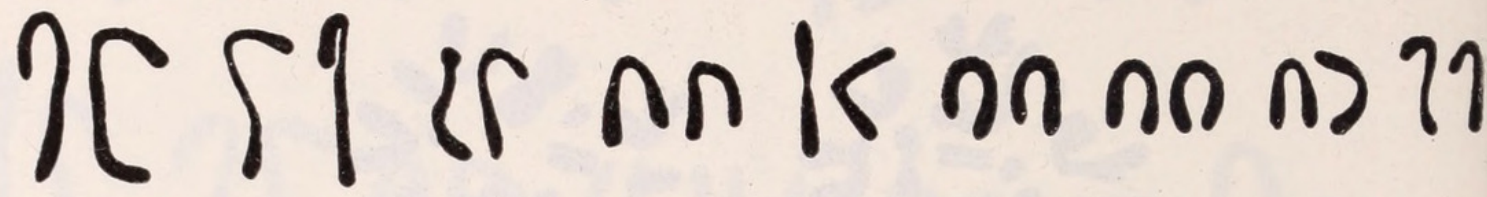

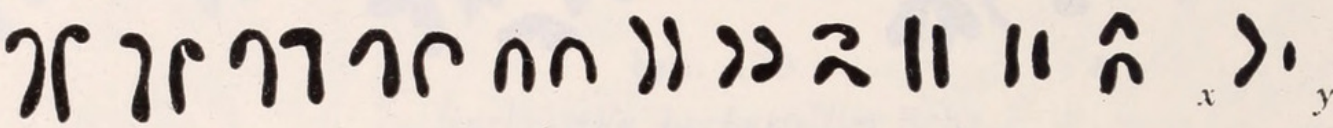

FIG. 118.

Nycteris sp.

Chromosomes spermatogoniaux. $(\times 4000$. $)$

forme, impair, représente probablement l' $Y$. Le nombre fondamental, égal à 79, est bien supérieur à celui des Rhinolophidae et des Vespertilionidae.

\section{DISCUSSION}

Arrivé au terme de l'exposé descriptif de la première partie de ce travail, j'aborderai quelques points particuliers pouvant donner lieu à une discussion: la question des hétérochromosomes, celle de l'évolution chromosomique, les relations entre la cytologie et la systématique.

\section{Les hétérochromosomes des Chiroptères.}

L'étude comparative des hétérochromosomes présente un intérêt d'autant plus grand que les cytologistes sont encore bien loin d'être unanimes en ce qui concerne leur interprétation.

Depuis les travaux de Painter (1921-1928), le type de digamétie $X Y$ a été reconnu chez un très grand nombre d'espèces de Mammifères, et le type $X O$ semble actuellement très exceptionnel dans cette classe. 
Les trois principaux critères qui permettent de déterminer à quel type de digamétie appartient une espèce donnée sont:

1. Le nombre diploïde de chromosomes, qui, s'il est impair, indique une digamétie $X O$, et s'il est pair, suggère soit l'existence d'un couple d'hétérochromosomes $X Y$, soit l'absence d'hétérochromosomes morphologiquement différenciés. Ce dernier dilemme sera en général résolu par l'appariement des chromosomes de l'assortiment diploïde.

2. L'examen des cinèses auxocytaires de profil, qui dans les cas favorables permet d'analyser la majorité des bivalents, et montre clairement la structure de l' $X Y$ lorsqu'il apparait en pré-réduction. Cette étude est grandement facilitée si les hétérochromosomes ont à ce stade une coloration différentielle. On peut alors suivre leur évolution tout au long de la méiose (Rhinolophus, Myotis).

3. L'étude des secondes cinèses de maturation, enfin, permet parfois de confirmer les renseignements acquis précédemment.

Ces critères ne sont certes pas à l'abri de toute critique. En ce qui concerne le premier point, il convient de remarquer que le décompte exact des chromosomes dans les métaphases diploïdes devient assez chimérique dès que l'on se trouve en présence de plus de quarante chromosomes, même avec le matériel le mieux fixé. Quant aux caryogrammes, on sait les nombreuses critiques dont ils ont fait l'objet: leur valeur démonstrative dépend essentiellement de la qualité des images dont ils sont tirés, de la précision du dessin, et du soin avec lequel ils sont établis. Il faut, en particulier, tenir compte de l'obliquité des chromosomes, au besoin rétablir leur longueur exacte, et confirmer par l'examen direct de la préparation les groupements par paires des chromosomes homologues. Les indications tirées des métaphases de première division de maturation en profil sont plus sûres. Toutefois, il faut encore se garder de conclure trop hâtivement: des bivalents autosomiques peuvent simuler un $X Y$ par leur aspect fortuitement dyssymétrique, et réciproquement, le couple $X Y$, s'il est en post-réduction, peut être pris pour une tétrade banale. D'autre part, on remarque souvent, à la métaphase de la première division, des chromatides isolées, ou des dyades précocement séparées, dont la présence en dehors de la plaque équatoriale pourrait suggérer une digamétie $X O$, alors que 
le schéma $X Y$ a été reconnu sans aucun doute chez l'espèce en question. Quant aux secondes divisions de maturation, leur fixation est si capricieuse et leur interprétation souvent si difficile, que les résultats de leur étude sont presque toujours incertains, à moins que les chromosomes sexuels ne soient hétérochromatiques.

Considéré isolément, aucun de ces critères n'est absolument sûr. Mais lorsque toutes ces indications se confirment et se complètent

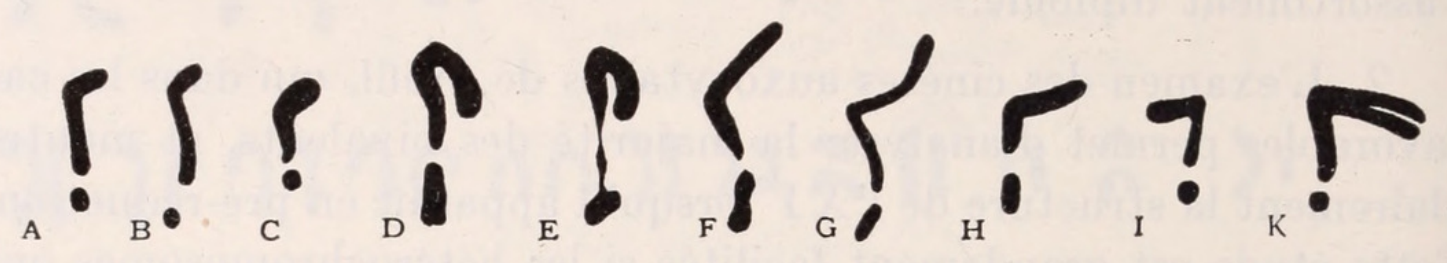

FIG. 119.

Aspect des hétérochromosomes dans les métaphases auxocytaires en profil chez les Chiroptères. A: Rhinolopus ferrum-equinum. B: Rh. euryale. C: Rh. hipposideros. D: Myotis myotis. E: M. daubentonii. F.: Pipistrellus pipistrellus. G: P. nathusii. H: Miniopterus schreibersii. I: Plecotus auritus. $\mathrm{K}$ : Barbastella barbastellus. $(\times 4000$.

mutuellement, il est alors possible d'établir avec certitude le type de digamétie de l'espèce en question.

En ce qui concerne les Chiroptères, nous avons constaté plus haut la présence, chez toutes les espèces étudiées, d'une paire d'hétérochromosomes $X Y$. Tenant encore compte des observations de Painter (1925) et de Makino (1948), on peut considérer ce type de digamétie comme général dans cet ordre, puisqu'il apparaît chez seize espèces, appartenant à cinq familles différentes.

La structure des hétérochromosomes témoigne d'une remarquable uniformité: sauf chez Rhinolophus hipposideros, l' $X$ est toujours un élément métacentrique de taille moyenne; ses deux bras sont presque égaux chez les Vespertilionidae, tandis que chez les Rhinolophidae l'un d'eux est environ trois fois plus long que l'autre. L'aspect du chromosome $X$ à la première cinèse de maturation est caractéristique de chaque genre (fig. 119). Chez Rhinolophus, le bras long de l' $X$ est en général tourné vers l' $Y$, parallèlement au fuseau. Chez Myotis, les deux bras de l' $X$ sont parallèles, et parfois accolés l'un à l'autre, tandis que chez Pipistrellus, ils sont largement divergents. Quant à l' $Y$, c'est toujours un élément très petit, le plus souvent punctiforme, mesurant rarement plus d'un $\mu$. 


\section{Liaison entre $X$ et $Y$ a la méiose.}

Les relations entre l' $X$ et l' $Y$ des Mammifères font l'objet de nombreuses controverses.

Les cytologistes japonais, Minouchi, Makino, Oguma, TAteishi, admettent un attachement de ces éléments par leurs extrémités, ou télo-synapsis. La nature de cette liaison "end to end" diffère donc fondamentalement de celle des autosomes: entre $X$ et $Y$, il n'y a pas de chiasmas, mais ces auteurs décrivent souvent une fine fibre chromatique reliant les deux constituants du bivalent sexuel. Enfin, durant les stades de l'accroissement, les hétérochromosomes sont enfermés dans une vésicule fortement colorée, qui disparaît à la diacinèse.

Les cytologistes anglais, Koller et Darlington (1934), Koller (passim) et ses élèves, défendent l'hypothèse d'une parasynapsis normale entre segments homologues (pairing segments) de l' $X$ et de l' $Y$, ce qui leur permet de donner une interprétation fort ingénieuse de la ségrégation des hétérochromosomes, qui peut être réductionnelle ou équationnelle à la première division de maturation. Leurs observations des stades préméiotiques ne concordent pas avec celles des cytologistes de l'école japonaise.

D'abord opposé à l'hypothèse de Koller et Darlington, MAтtнey s'y est rallié plus tard $(1938,1947)$, avec quelques réserves relatives aux stades préméiotiques, et en insistant sur le fait que cette théorie, féconde au point de vue génétique, reposait sur des bases cytologiques fragiles. Il est probable que l'étude actuellement en cours des chromosomes sexuels géants de Microtus agrestis lui permettra d'élucider définitivement ce problème (МАттне Y 1949 a).

Chez les Chiroptères, la taille relativement petite des hétérochromosomes, et particulièrement de l' $Y$, ne favorise pas l'étude de ce point particulier. Voici cependant les observations que j'ai pu faire.

Stades pré-mé i o tiques: Leur analyse confirme les observations de Matтhey et des auteurs japonais, en ce qui concerne l'existence d'un hétérocaryosome, renfermant les chromosomes sexuels. Toutefois, sa présence est loin d'être constante, et les filaments hétérochromosomiques apparaissent souvent libres de toute enveloppe, ou recouverts seulement d'un dépôt pycnotique 
plus ou moins épais, d'une opacité variable. L'hétérocaryosome n'est donc pas une vésicule au sens strict, mais un simple dépôt d'une substance basophile sur les chromosomes sexuels, formant une gouttelette à l'intérieur de laquelle ces derniers restent emprisonnés grâce à l'action des tensions superficielles. De la quantité plus ou moins grande de substance déposée dépend la formation, ou au contraire l'absence de "vésicule". J'ai observé au stade pachytène les figures caractéristiques en 8 et en $\mathrm{S}$ décrites par de nombreux auteurs (Minouchi, 1928; Oguma, 1935; Matthey, 1936 et 1938; Макімо, 1941), mais en aucun cas je n'ai pu déceler l'indice certain d'une parasynapsis entre $X$ et $Y$, qui apparaissent soit séparés nettement, soit unis par leurs extrémités (cf. Myotis myotis et $M$. daubentonii).

Première division d e maturation: A la métaphase, l' $X$ et l' $Y$ sont souvent déjà séparés, alors que les constituants des tétrades autosomiques sont encore unis. La petite taille de l' $Y$ est probablement responsable de cette précocité, car chez Myotis où ce chromosome est plus long, on rencontre plus fréquemment des figures où les deux chromosomes sexuels sont encore réunis. La zone de jonction est souvent marquée par un épaissisement, et, de part et d'autre de cette sorte de nœud, les chromatides paraissent étirées (fig. 50 et 67). En fait, le chromosome $Y$ ainsi sous tension est sensiblement plus long qu'après la rupture des liaisons. Dans de nombreux cas enfin, on ne voit, entre l'extrémité de l' $X$ et l' $Y$, qu'une zone où le protoplasme est plus foncé: il est alors difficile de juger s'il s'agit d'une structure réelle ou d'un artifice de coloration.

Remarquons enfin que si, chez les Vespertilionidae, le chromosome $Y$ est toujours dans le prolongement de l'un des bras de l' $X$, il n'en est pas toujours de même chez les Rhinolophidae (fig. 9, 23, 36). J'ai déjà dit plus haut (cf. p. 384) comment on pouvait expliquer ce fait.

Mes observations ne s'accordent entièrement ni avec l'hypothèse de Koller et Darlington, ni avec les conceptions des cytologistes japonais. Alors que l'étude des stades pré-méiotiques semble donner raison à ces derniers, en ne montrant pas de parasynapsis nette, les métaphases auxocytaires indiquent en revanche des arguments favorables à l'hypothèse d'une liaison chiasmatique, et 
montrent en tout cas que l'attachement "end to end " n'est qu'un des stades de l'évolution des hétérochromosomes.

\section{Pré-réduction et post-réduction.}

Toutes les figures d'hétérochromosomes de profil que j'ai observées, sauf une, montrent l' $X$ et l' $Y$ en pré-réduction. De là à l'affirmation que la post-réduction n'existe pas chez les Chiroptères, il n'y a qu'un pas; il convient cependant d'être prudent.

Chez les Rhinolophidae en particulier, l'hétérochromatie généralement bien marquée du couple $X Y$, ainsi que son aspect très caractéristique, permettent de déceler très facilement les cas de pré-réduction. Toutefois, quelques métaphases ne m'ont pas montré le moindre signe trahissant la présence d'hétérochromosomes, ceci malgré une excellente fixation, et une coloration très bien réussie. Deux explications se présentent à l'esprit: la première consiste à admettre qu'une association en post-réduction aboutit à la formation d'un bivalent d'aspect autosomique ne manifestant pas d'hétérochromatie, et dès lors très difficile à identifier; la seconde suppose que l' $X Y$, en position "couchée», et peut-être non hétérochromatique, est masqué par d'autres bivalents. La figure 27 montre un cas tout aussi douteux: l'élément placé en dehors de la plaque équatoriale rappelle les figures de post-réduction d'Epimys norvegicus et des Apodemus (Маттнеу, 1936, 1938, 1947); mais si l'on se réfère aux figures 9 et 23 de ce présent travail, on peut, avec autant de vraisemblance, l'interpréter comme un cas de préréduction, et admettre que l' $Y$ est encore intimement lié à la partie proximale de l' $X$, de couleur plus sombre.

Chez les Vespertilionidae, les faits sont plus clairs, et la préréduction est constante.

En résumé, l'existence de la post-réduction est improbable chez les Chiroptères, ou, si elle existe, elle n'affecte qu'une proportion infime d'auxocytes.

\section{Hétérochromatie des Chromosomes Sexuels.}

Au cours des divisions méiotiques, l'hétérochromatie des chromosomes sexuels n'est pas constante, et dépend probablement de la fixation, car dans la même coupe, il n'est pas rare de trouver 
des différences très grandes entre deux zones parfois très proches. Chez les Chiroptères, il s'agit toujours d'une hétérochromatie positive, qui n'apparaît, dans le matériel coloré à l'hématoxyline ou au violet cristal, que si la différenciation est assez poussée, après décoloration presque totale des autosomes. Le contraste s'accentue encore à l'anaphase, et dans les noyaux issus de la première division de maturation les hétérochromosomes sont en général bien colorés, alors que les autosomes sont pratiquement invisibles. A la seconde division, l'hétérochromatie est beaucoup plus rare, sauf à la télophase. Enfin, nous avons déjà remarqué que l' $X Y$ préméiotique était fortement hétérochromatique.

Les auteurs donnent en général peu de renseignements sur la question qui nous intéresse ici. En ce qui concerne les Chiroptères, Painter (1925) n'a étudié que des mitoses diploïdes chez Nyctinomus mexicanus, et Makino (1948) ne fait aucune allusion à ce sujet dans sa description des chromosomes de Rhinolophus ferrumequinum nippon. Chez les autres Mammifères, on rencontre égale-

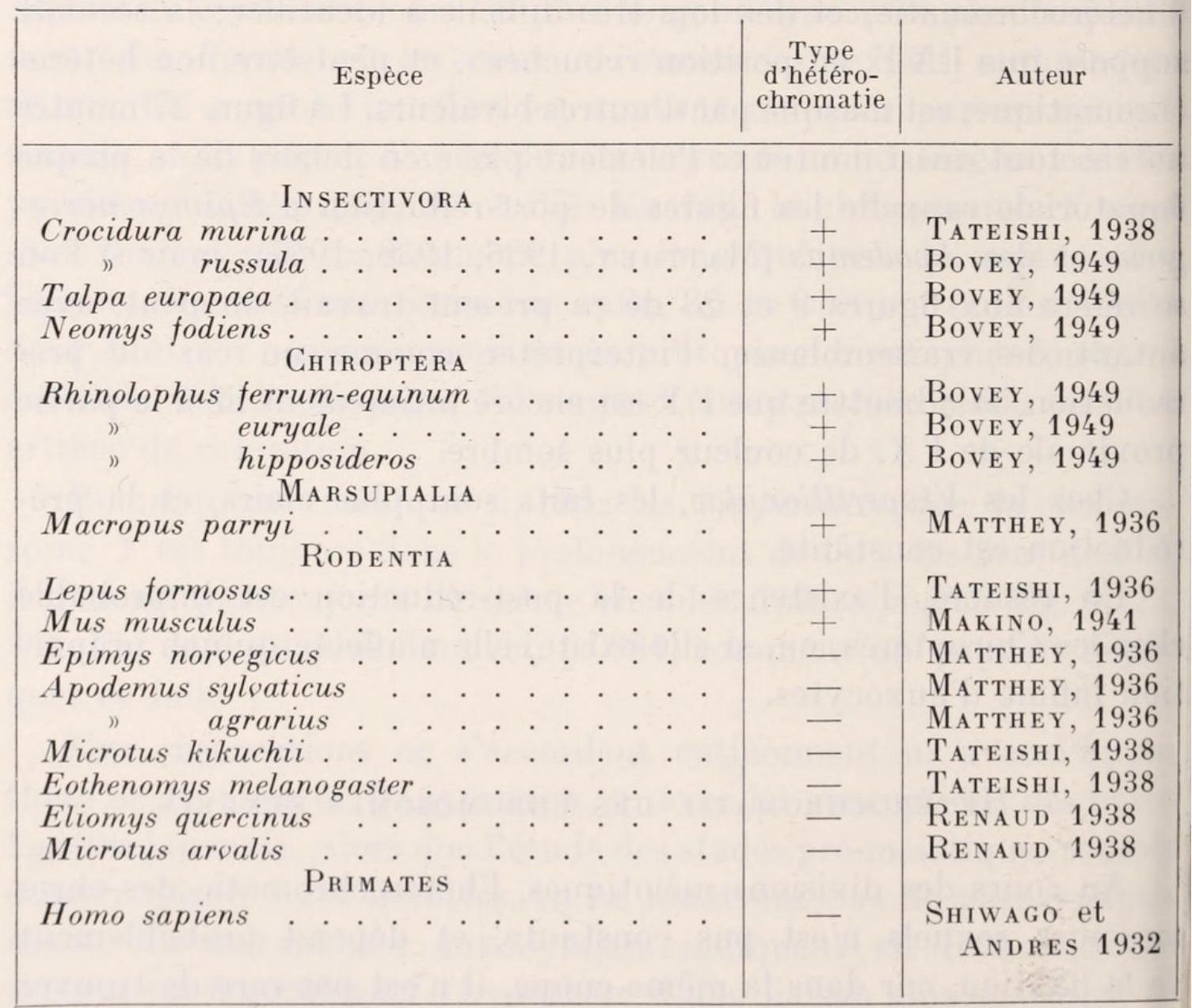


ment l'hétérochromatie positive et négative, mais des données précises à ce sujet sont rares. Voici celles que j'ai pu recueillir: elles concernent l'état du bivalent sexuel à la première division de maturation. (cf. table p. 416).

Les Chiroptères, les Insectivores, les Marsupiaux et une partie des Rongeurs ont le même type d'hétérochromatie. Chez les Chiroptères, l'hétérochromatie n'est certaine, à la première division, que chez les Rhinolophidae.

\section{Evolution chromosomique chez les Chiroptères.}

Considéré dans son ensemble, le groupe des Chiroptères paraît très peu homogène, si l'on compare entre eux les nombres diploïdes, qui varient de 32 chromosomes (Plecotus, Barbastella) à 58 (Rhinolophus).

$\mathrm{Si}$, en revanche, on tient compte du nombre de bras chromosomiques ou nombre fondamental (N.F.), on arrive à des résultats très différents, qui mettent en évidence la remarquable unité cytologique existant au sein des deux familles des Rhinolophidae et des Vespertilionidae. L'hypothèse de Roвевтson (1916), reprise et développée par Matтhey $(1931,1933,1945,1949)$ et White (1945), selon laquelle un chromosome métacentrique peut se transformer, au cours de l'évolution, en deux acrocentriques, et réciproquement, suivant l'équation $V \rightleftarrows I+I$, trouve ici encore une excellente justification.

Le tableau suivant (p. 418), établi en tenant compte de mes résultats et de ceux de Makino (1948) et Painter (1925), montre que les processus robertsoniens ont joué un rôle certain dans l'évolution des Chiroptères.

Ces observations s'accordent-elles avec les hypothèses que la Paléontologie et l'Anatomie comparée permettent d'envisager quant à l'origine et l'évolution des Chiroptères? Et si nous admettons que l'évolution chromosomique des Chiroptères s'est faite en partie selon le schéma de Robertson, dans quel sens ces processus ont-ils agi ? Fragmentation de chromosomes, ou au contraire fusions génératrices de $V$ ?

Chez les Vertébrés inférieurs, comme l'a montré Matтhey $(1945,1949$ b) l'abondance de chromosomes télocentriques et 


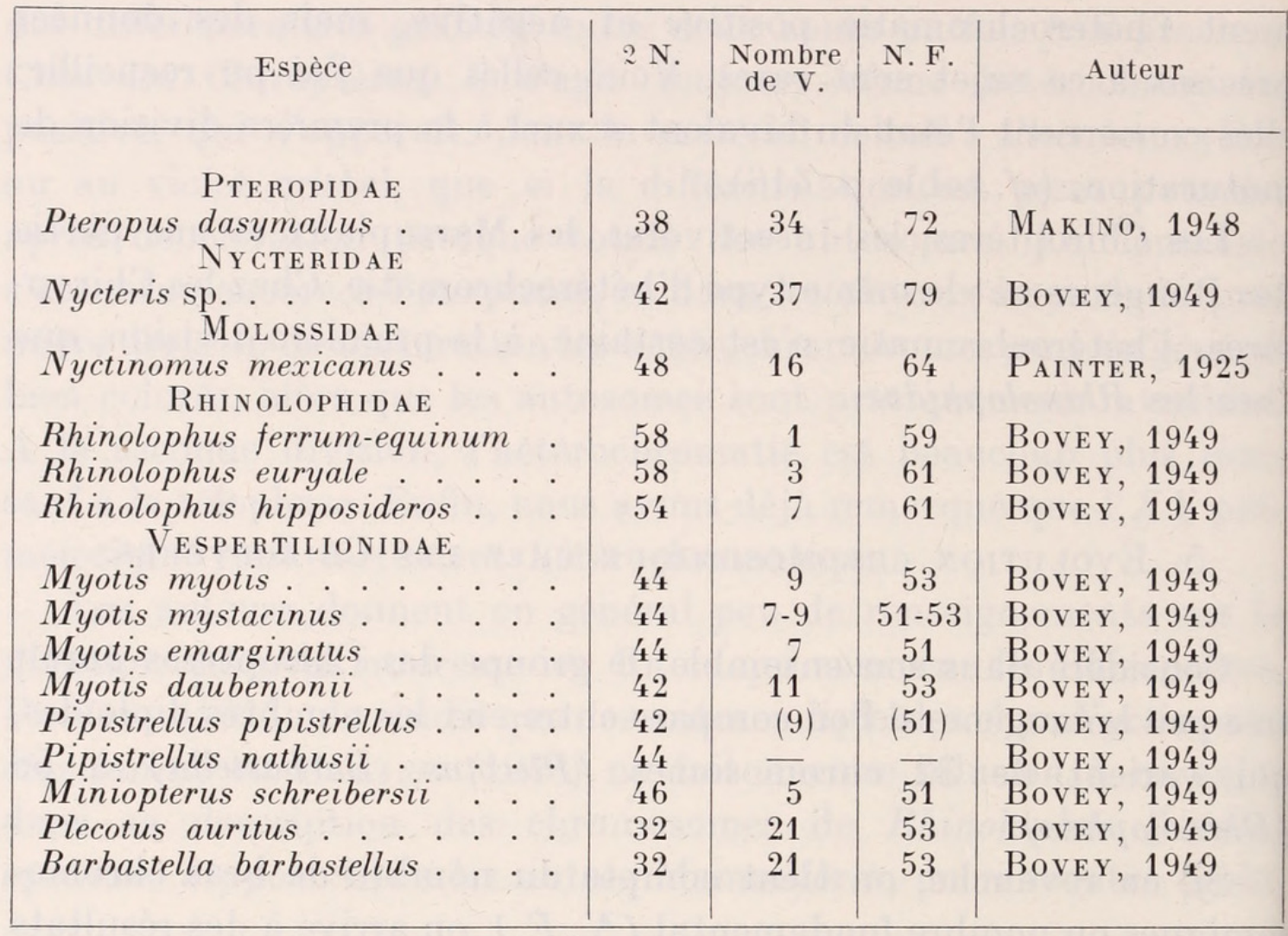

l'absence ou le petit nombre de $V$ constituent des caractères primitifs. En est-il de même chez les Chiroptères ?

Bien que les fossiles soient plutôt rares, on sait que des formes très proches des Chiroptères actuels existaient déjà au début de l'ère tertiaire. Les gisements de phosphorite du Quercy, datant de la fin de l'Eocène, contiennent des restes de Rhinolophidae, de Vespertilionidae, de Molossidae et d'autres formes actuellement éteintes. Si l'on s'en rapporte à la répartition stratigraphique indiquée par Revilliod (1922) pour les genres fossiles du tertiaire, on peut admettre que les Rhinolophidae et les Phyllostomatidae, présents dès le début de l'Eocène, sont antérieurs aux Vespertilionidae, aux Emballonuridae et aux Nycteridae. Le genre Myotis, par exemple, n'apparaît pour la première fois qu'à l'Oligocène supérieur, et la famille des Vespertilionidae se développe surtout au Miocène.

Il est tentant de rapprocher ces données de nos observations cytologiques. Les Rhinolophidae, pauvres en chromosomes métacentriques et possédant un nombre diploüde élevé, seraient donc des formes primitives, tandis que les Vespertilionidae, plus riches en $V$, seraient plus évolués, de même que les Nycteridae et les Ptero- 
pidae. Ainsi, les phénomènes de fusions centriques auraient été prédominants au cours de l'évolution des Chiroptères.

Les données de l'Anatomie comparée, en revanche, semblent contredire cette dernière hypothèse. En effet, les Pteropidae, dont l'assortiment se compose presque uniquement de chromosomes métacentriques, et que l'on serait en droit, d'après ce que nous avons vu plus haut, de considérer comme très évolués, possèdent des caractères physiologiques primitifs (WEBER, 1928). Il en est de même pour le genre Nycteris, très riche en métacentriques, et que WeBER considère comme un genre très primitif.

Les paléontologistes admettent généralement que les Chiroptères dérivent d'une souche d'Insectivores arboricoles, ou tout au moins qu'ils ont avec cet ordre une origine commune. Je parlerai, dans la seconde partie de ce travail, des indications que la cytologie peut apporter à l'appui de cette hypothèse.

Concluons: les processus d'évolution conformes à la loi de Robertson sont évidents chez les Chiroptères. Mais le sens de cette évolution est difficile à préciser, en raison du petit nombre de documents, tant paléontologiques que cytologiques.

\section{Systématique et chromosomes.}

Bien que le nombre d'espèces étudiées au point de vue cytologique soit encore très petit, on peut déjà noter, en ce qui concerne les deux familles des Rhinolophidae et des Vespertilionidae, la concordance remarquable entre la classification systématique d'une part, et les données de la cytologie d'autre part.

$\mathrm{R} h$ in ol o p.hidae: Cette famille, très homogène, montre des caractères cytologiques bien nets: grand nombre d'éléments acrocentriques, plaque équatoriale très dense, chromosome $X$ nettement hétérochromatique, à bras inégaux, entre eux dans le rapport 4:1 environ; nombre fondamental $=59-61$. La parenté étroite entre Rhinolophus euryale et $R h$. ferrum-equinum se manifeste dans l'identité de leurs garnitures chromosomiques, tandis que Rhinolophus hipposideros se distingue des deux espèces précédentes par un nombre diploïde moins élevé.

V e s p e rtilio nida e: Le nombre fondamental reste très constant, puisqu'il ne varie qu'entre 51 et 53 , et caractérise la

Rev. Suisse de Zool., T. 56, 1949. 
famille. La comparaison des nombres diploïdes entre eux, et celle des hétérochromosomes, donne un argument en faveur de la division de cette famille en trois sous-familles: Vespertilioninae, Plecotinae, Miniopterinae, - cette dernière différant peu des Vespertilioninae au point de vue cytologique. - L'identité complète des garnitures chromosomiques des deux Plecotinae étudiés ici, Plecotus auritus et Barbastella barbastellus, est très intéressante, car ces deux espèces ont des formules dentaires différentes. En revanche, Pipistrellus pipistrellus et $P$. nathusii, souvent difficiles à distinguer morphologiquement, n'ont pas la même formule chromosomique, nathusii ayant deux chromosomes de plus que pipistrellus. Notons enfin que Myotis myotis, M. mystacinus et M. emarginatus ont le même nombre de chromosomes, fait qui pourrait justifier la classification, adoptée parfois, de mystacinus comme sous-espèce de Myotis myotis.

Nycteridae: Parmi toutes les Chauves-souris étudiées jusqu'à maintenant, c'est le genre Nycteris qui possède à la fois le plus grand nombre de $V$ (37), et le $N$. F. le plus élevé (79). Ce fait permet d'esquisser un rapprochement de cette famille avec celle des Pteropidae, dont les représentants sont aussi très riches en éléments métacentriques, et ont un $N . F$. élevé, si l'on en juge d'après Pteropus dasymallus, étudié par Maкino (1948).

\section{DEUXIÈE PARTIE}

\section{LES CHROMOSOMES DES INSECTIVORES}

Les relations phylétiques certaines existant entre les Insectivores et les Chiroptères m'ont encouragé à mener de front l'étude cytologique de ces deux ordres primitifs de Mammifères et à publier mes résultats dans un seul travail, afin de pouvoir établir des comparaisons et tirer si possible des conclusions générales. Je suivrai le même plan que pour la première partie, mais je donnerai une plus grande importance au cas de l'une des Musaraignes étudiées, Sorex araneus L., en raison de l'intérêt que présentent ses chromosomes sexuels. 


\section{HISTORIQUE}

Il n'existe jusqu'à maintenant que trois travaux consacrés aux chromosomes des Insectivores. Le premier est de Painter (1925) qui, étudiant les mitoses de l'amnios du Hérisson (Erinaceus europaeus L.) trouve 48 chromosomes, nombre que j'ai pu confirmer, mais ne réussit pas à mettre en évidence les chromosomes sexuels. La seconde contribution est due à Koller (1936). Analysant la spermatogenèse de la Taupe, cet auteur fixe le nombre diploïde à 38 , avec digamétie de type $X Y$ et pré-réduction constante. Enfin, plus récemment, TAтеishi (1938) a étudié deux Insectivores asiatiques: une Taupe, Mogera insularis Swinhoe, qui compte 32 chromosomes, et une Musaraigne, Crocidura murina L., chez laquelle le nombre diploïde est égal à 40. Chez ces deux espèces, Tateishi note une digamétie ô de type $X Y$.

J'ai publié deux notes préliminaires, l'une concernant Sorex araneus L. (Bovey 1948), l'autre résumant les observations faites sur les cinq espèces d'Insectivores que j'ai étudiées (Bovey 1949).

\section{MATÉRIEL}

Je n'ai pas eu de peine à me procurer des Hérissons, dont j'ai aussi fait l'élevage en captivité, afin d'avoir des mâles nouveaunés. Un taupier m'a procuré des Taupes vivantes. Quant aux Musraignes, leur capture m'a donné quelque difficulté. Leur abondance

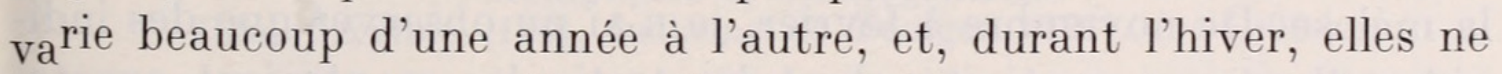
sortent que fort peu. J'ai utilisé pour les capturer vivantes des souricières en treillis, de modèle courant, en mettant comme appât du lard, de la viande crue renouvelée chaque jour. En hiver ou au printemps, les petits Mammifères capturés meurent de froid au bout de quelques heures, si l'on n'a pas soin de visiter les pièges à intervalles réguliers, toutes les deux ou trois heures.

Au cours de l'hiver 1948-1949, j'ai élevé, sans grande difficulté, 3 ○ิ ô de Crocidura russula en les nourrissant de viande crue hachée. 
LISTE DES ESPÈCES ÉTUDIÉES

\begin{tabular}{|c|c|c|c|}
\hline Espèces & $\begin{array}{c}\text { Adul- } \\
\text { tes }\end{array}$ & Jeunes & Localité \\
\hline Erinaceus europaeus L. . & 6 & 4 & Lausanne et environs \\
\hline Talpa europaea L. . . . & 7 & - & Lausanne et environs \\
\hline Neomys fodiens Pall. . & 2 & - & Allaman \\
\hline Crocidura russula Herm. . . . . & 10 & - & Lausanne et environs \\
\hline Sorex araneus L. . . . . . . . . & 2 & 一 & $\begin{array}{l}\text { Romanel s/Lausanne, } \\
\text { Gryon s/Bex }\end{array}$ \\
\hline
\end{tabular}

Les fixations ont en général bien réussi, sauf chez la Taupe où deux individus seulement m'ont donné des résultats satisfaisants.

\section{LE CYCLE SPERMATOGÉNÉTIQUE DES INSECTIVORES}

Courrier (1927) a étudié l'évolution de la spermatogenèse et des caractères sexuels chez Talpa europaea et Erinaceus europaeus, et montré que chez ces deux espèces, le testicule présente un cycle évolutif annuel. Le maximum d'activité a lieu en mars chez la Taupe et en avril-mai chez le Hérisson. En été, le testicule est au repos chez Talpa, l'activité méiotique cessant complètement. Chez Erinaceus, la spermatogenèse dure plus longtemps et ne cesse qu'au début de l'automne.

Mes observations sont en accord avec celles de Courrier, mais comme cet auteur n'a pas étudié le cycle des Musaraignes, je crois qu'il peut être utile d'en dire quelques moṫs: chez Crocidura russula, le cycle saisonnier est très peu marqué, ou peut-être même inexistant. Dès la fin de février et jusqu'en novembre, la spermatogenèse est continue, et l'on trouve alors tous les stades caractéristiques de la méiose. De novembre à février, je n'ai pu observer que des individus élevés en captivité, dont les testicules montraient exactement le même aspect histologique que ceux qui avaient été fixés en été ou au printemps. Matthey (inédit) a examiné le testicule d'une Crocidura capturée au mois de janvier. Il n'a vu que des divisions spermatogoniales, ce qui semble indiquer une reprise d'activité à ce moment. Ainsi, le cycle annuel de Crocidura russula n'est marqué que par un très bref arrêt en hiver, probablement de la fin de novembre au début de janvier. Chez Sorex araneus et 
Neomys fodiens, je n'ai pris des mâles qu'au printemps. A ce moment, la spermatogenèse est intense, et l'on trouve tous les stades.

\section{OBSERVATIONS CYTOLOGIQUES}

1. Famille des Erinaceidae.

\section{Erinaceus europaeus L.}

M a térie l: 6 ô ô adultes (mars, avril, mai, juillet) et

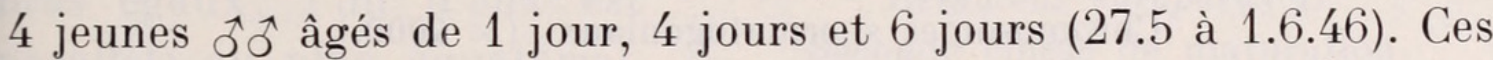
derniers m'ont fourni de belles figures de métaphases spermatogoniales.

O b s e r v a ti o $\mathrm{ns}$ : Les divisions spermatogoniales (fig. 120 et 121) sont de grande taille chez les nouveau-nés. La plaque équa-

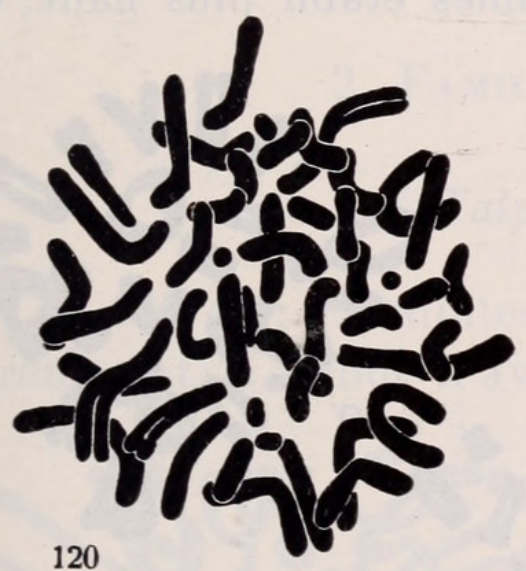

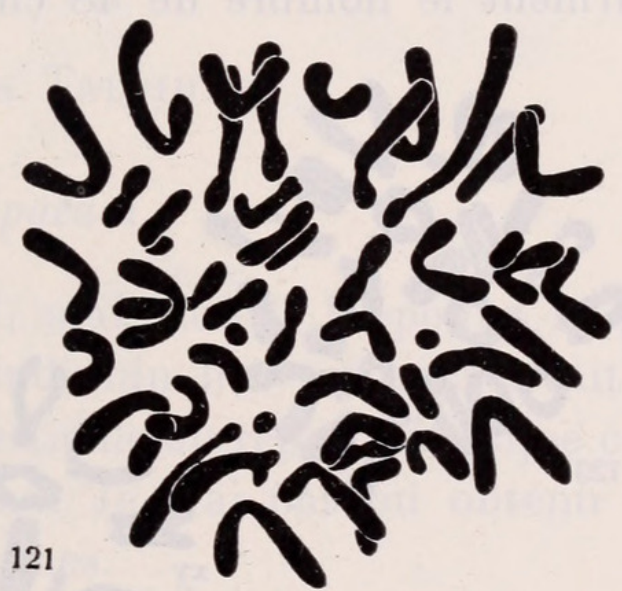

121

FIG. 120 et 121.

Erinaceus europaeus L.

Métaphases spermatogoniales.

Hématoxyline (120), fuchsine de Feulgen (121). ( $\times$ 4000.)

toriale présente 48 chromosomes, parmi lesquels les métacentriques, au nombre de 39 , prédominent largement. On trouve en outre 4 éléments acrocentriques, 4 punctiformes, et une paire d'hétérochromosomes formée d'un $X$ métacentrique de taille moyenne, et d'un $Y$ punctiforme, tous deux facilement mis en évidence dans les caryogrammes (fig. 122). Le nombre fondamental peut être évalué à 87. 
Les noyaux des auxocytes ont la même structure que chez les Chiroptères: outre les filaments autosomiques, on y remarque une

\section{(j)

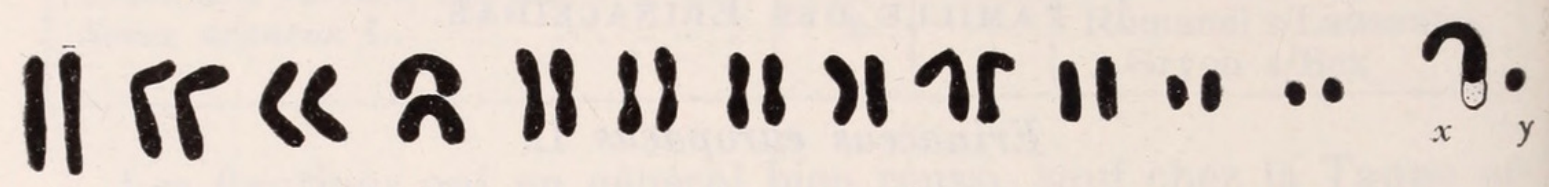

FIG. 122.

Erinaceus europaeus L.

Chromosomes spermatogoniaux. $(\times$ 4000.)

grosse masse très chromophile, appliquée contre la membrane nucléaire, et contenant les chromosomes sexuels. Je n'ai pas observé de diacinèses.

Les métaphases de première division de maturation (fig. 123-127) confirment le nombre de 48 chromosomes établi plus haut; elles

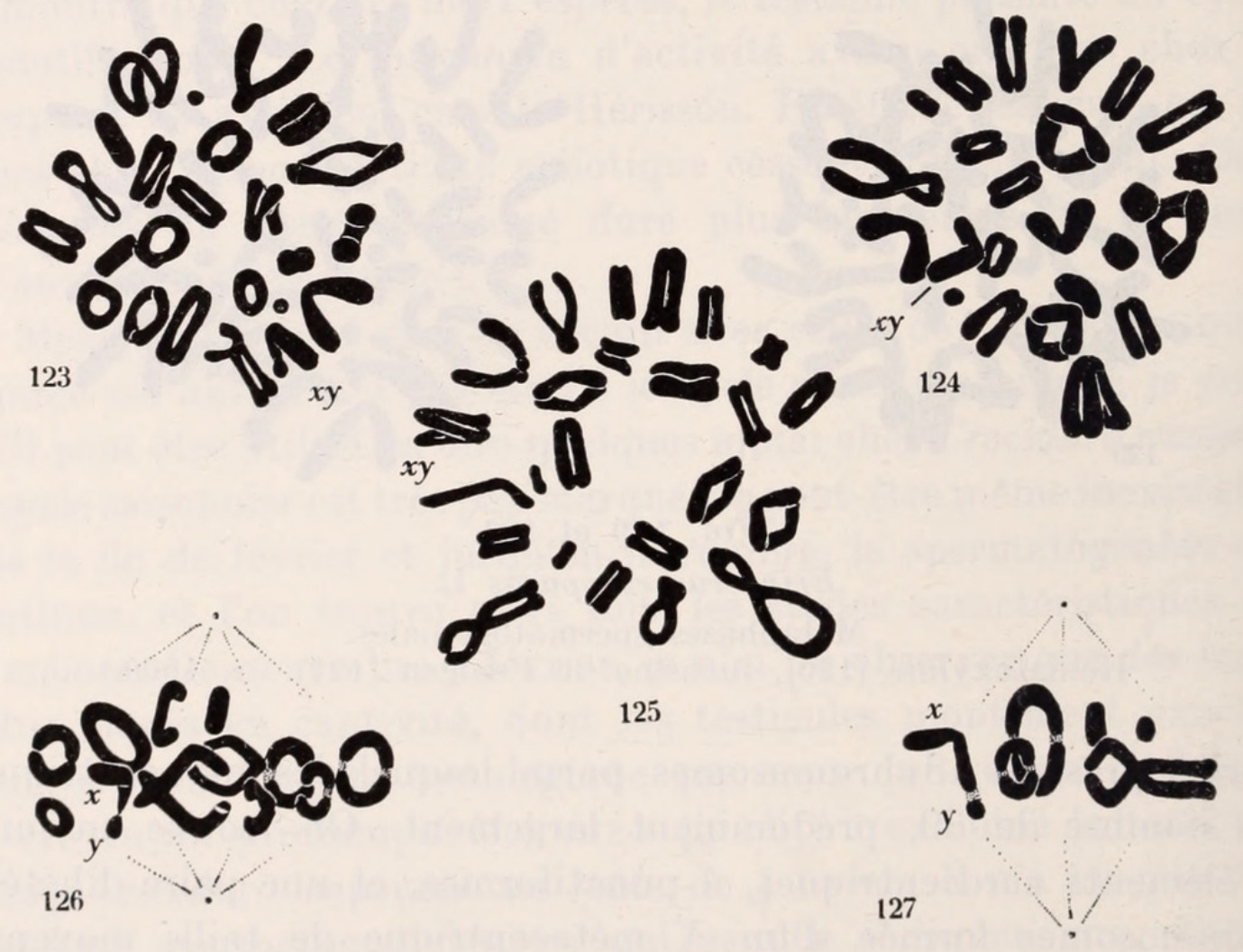

FIG. 123 à 127.

Erinaceus europaeus L.

Métaphases auxocytaires, en vue polaire $(123-125)$ et de profil $(126,127)$. Hématoxyline. $(\times 4000$. $)$ 
contiennent en effet 24 bivalents de formes diverses. La majorité des éléments métacentriques ont leurs chiasmas terminalisés, et forment des anneaux perpendiculaires au plan équatorial. D'autres bivalents dessinent des 8 ou des bâtonnets. On reconnait facilement, dans les métaphases en vue polaire, et surtout dans les figures vues de profil (fig. 126 et 127) le couple hétéromorphe $X Y$. Le chromosome $X$ a deux bras égaux; l'un d'eux est parallèle au plan équatorial, et l'autre, plus ou moins perpendiculaire, est prolongé par un petit $Y$ punctiforme. La pré-réduction est constante.

Seconde division de maturation: 24 dyades (fig. 128 et 129).

\section{Famille des Talpidae.}

\section{Talpa europaea L.}

M a t é r i e l: 6 adultes (avril, mai, août). L'époque la plus favorable est le début d'avril. L'individu fixé en août avait un

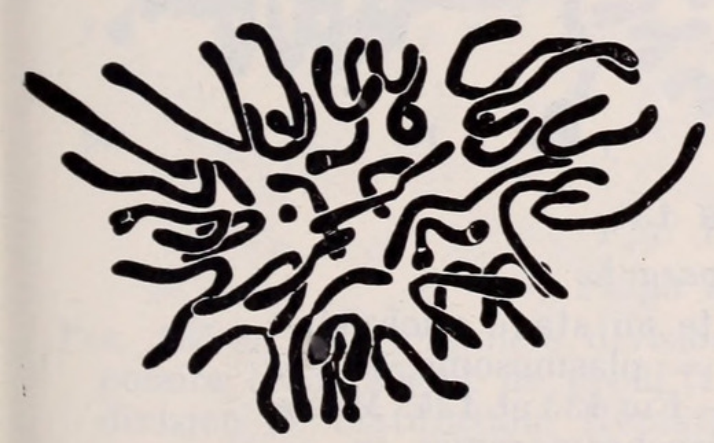

FIG. 130.

Talpa europaea L.

Métaphase spermatogoniale. Hématoxyline. $(\times 4000$. $)$ testicule au repos mitotique complet. Je n'ai pas pu obtenir des jeunes.

O b s e r v a t i o n s : Les divisions spermatogoniales sont rarement très bien fixées, et leur aspect (fig. 130) montre d'emblée que tous les chromosomes sont métacentriques, à l'exception d'un seul petit élément punctiforme, qui est évidemment l' $Y$. La sériation des 34 chromosomes spermatogoniaux est difficile, en raison $\mathrm{du}$ fait que tous les grands métacentriques sont flexueux, et fort semblables. Celle que j'ai établie (fig. 131) me conduit à 
considérer comme chromosome $\mathrm{X}$ un élément métacentrique à bras inégaux, qui se distingue des autres par un aspect plus rigide. Le nombre fondamental est 67.

Les noyaux pachytènes (fig. 132-134) contiennent un volumi-
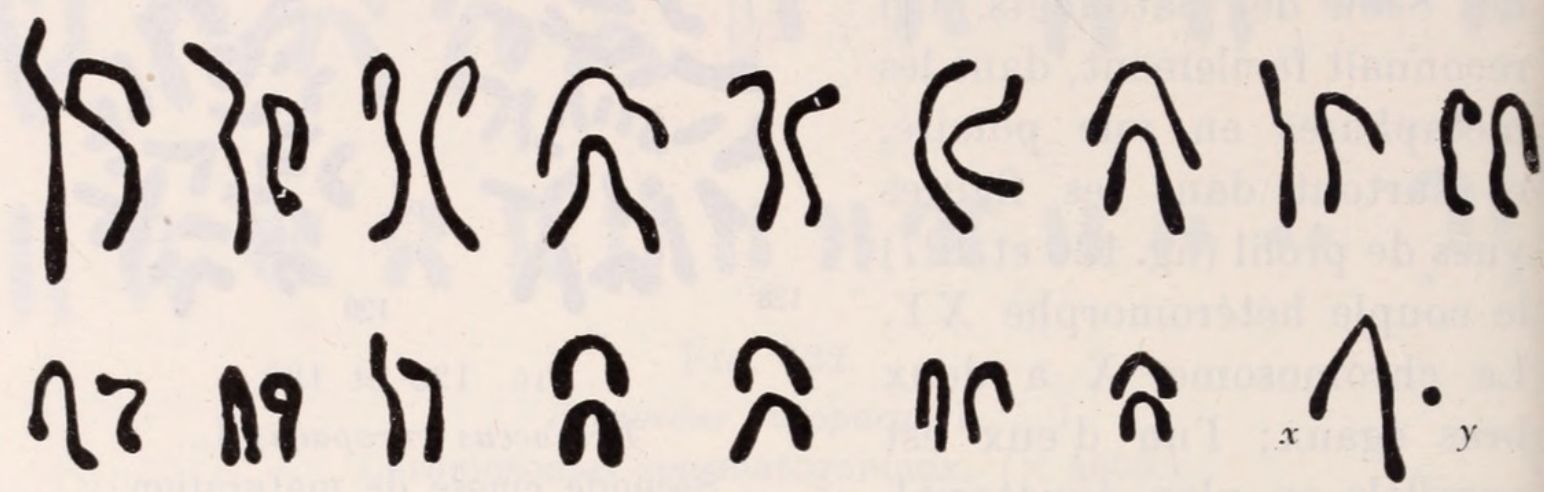

FIG. 131.

Talpa europaea L.

Chromosomes spermatogoniaux. $(\times 4000$. $)$

neux plasmosome, plusieurs nucléoles, et un filament hétérochromatique de forme complexe et variable, formé par l'union en para-

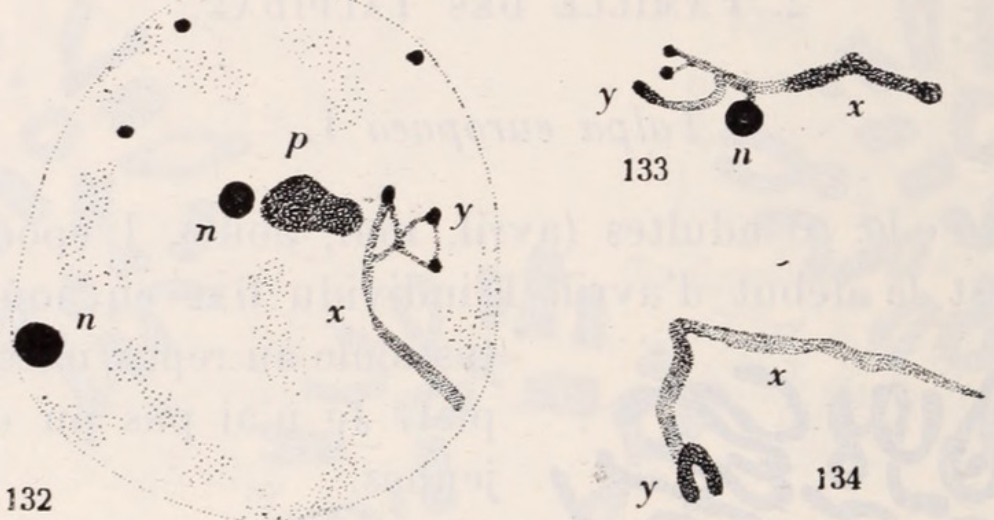

FIG. 132 à 134.

Talpa europaea L.

Fig. 132. Noyau d'auxocyte au stade pachytène (diffus) $; n=$ nucléole, $p=$ plasmosome, $x$ et $y$ $=$ hétérochromosomes. - FIG 133 et 134. Même stade, aspect des hétérochromosomes. $n=$ nucléole. Hématoxyline. $(\times 4000$. $)$

synapsis de l'X et de l'Y. Les segments appariés des deux chromosomes sexuels sont terminaux.

Les métaphases des premières divisions de maturation (fig. 135141), très semblables à celles du Hérisson, se composent de 17 bivalents, parmi lesquels le couple $X Y$ se distingue soit par sa 
forme dyssymétrique, soit par son hétérochromatie positive, lorsque les autosomes sont assez décolorés (fig. 136). On remarque aussi la présence d'un nucléole arrondi, légèrement plus gros que le chromosome Y; placé souvent dans le plan équatorial, il peut
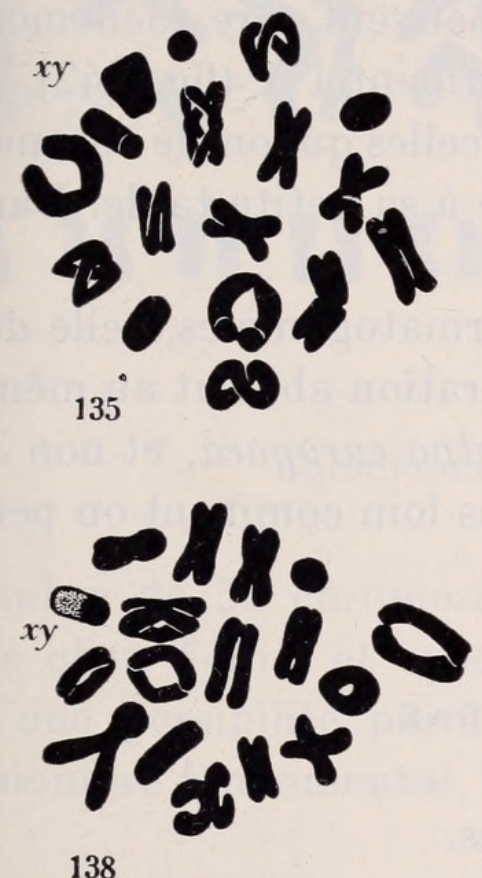

138

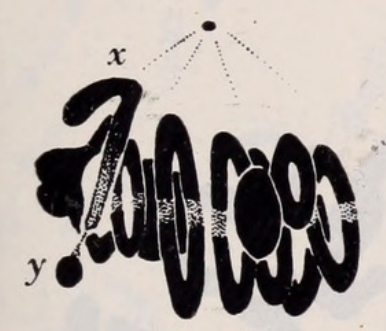

140
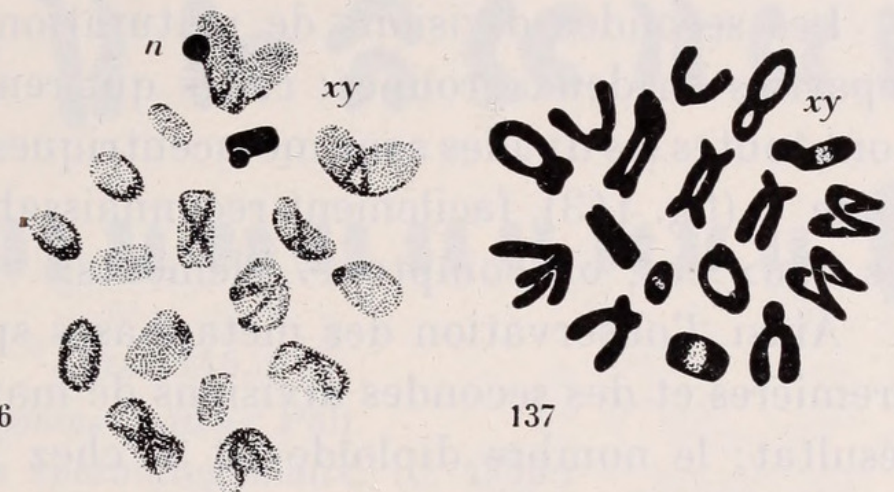

137

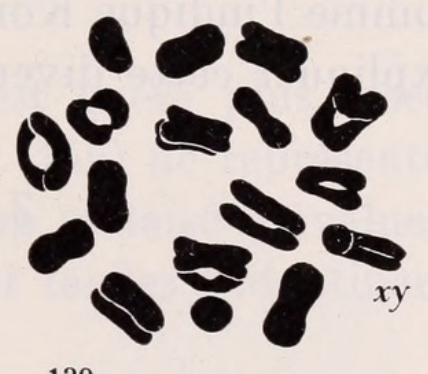

139

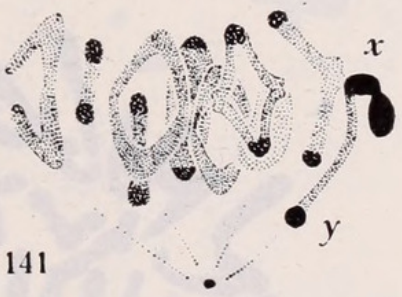

FIG. 135 à 143.

Talpa europaea L.

FIg. 135 à 141. Première division de maturation. Métaphases en vue polaire (135-139) et de profil $(140,141)$. - Fig. 142 et 143. Seconde division de maturation. Hématoxyline. $(\times 4000$.

être une cause d'erreur de numération. Les métaphases de profil (fig. 140 et 141) montrent à la fois la structure des bivalents autosomiques et celle du couple $X Y$. Les premiers ont en général l'aspect d'un anneau étiré dans le sens de la traction fusorielle, et une zone plus claire, au niveau du plan équatorial, trahit la présence des liaisons dont la rupture est imminente (fig. 140). Quant au 
couple $X Y$, il a la même disposition que chez le Hérisson, mais les deux bras de l' $X$ sont inégaux (rapport 2: 1 environ); le bras court est parfois divisé en deux segments par une constriction médiane (fig. 141) et le bras long, qui paraît souvent étiré, est en général moins coloré que le bras court. Il y a toujours pré-réduction.

Les secondes divisions de maturation peuvent être facilement réparties en deux groupes: celles qui renferment l' $X$ (fig. 142), et dont toutes les dyades sont métacentriques; celles qui ont le chromosome Y (fig. 143), facilement reconnaissable à sa petite taille. Dans les deux cas, on compte 17 éléments.

Ainsi, l'observation des métaphases spermatogoniales, celle des premières et des secondes divisions de maturation aboutit au même résultat: le nombre diploïde est 34 chez Talpa europaea, et non 38 comme l'indique Koller. Nous verrons plus loin comment on peut expliquer cette divergence.

\section{Famille des Soricidae.}

Neomys fodiens Pallas.

M a térie l: 2 ô ô adultes (20.3.48) chez lesquels j'ai pu observer tous les stades de la spermatogenèse.
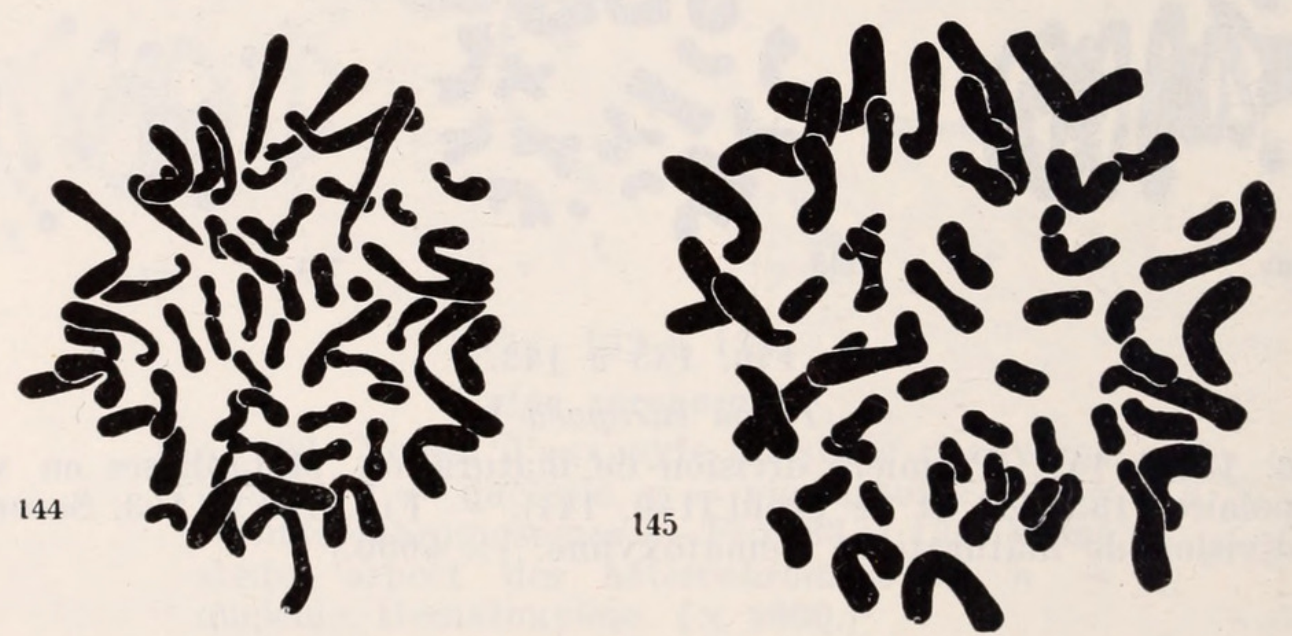

FIG. 144 et 145.

Neomys fodiens Pall.

Métaphases spermatogoniales.

Trichromique de Flemming (144), hématoxyline (145). ( $\times$ 4000.)

O b s e r v a t i o n s: Le nombre élevé des chromosomes et la forte proportion de métacentriques attirent immédiatement l'atten- 
il n'en est pas de même pour les éléments plus petits, chez lesquels la position centromérique n'apparaît pas clairement. Il semble cependant qu'il y ait au moins 40 chromosomes en $V$, ce qui donne un nombre fondamental de 92 ,

Durant le stade pachytène et le stade diffus qui lui fait suite, on aperçoit dans le noyau, parmi les autosomes flous et pâles, l'hétérocaryosome dont la coloration est généralement intense, et qui reste appliqué contre la membrane nucléaire. Il est rarement
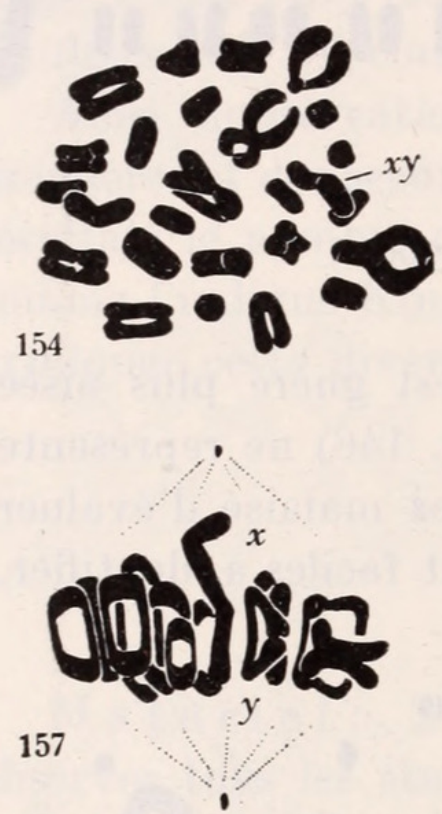
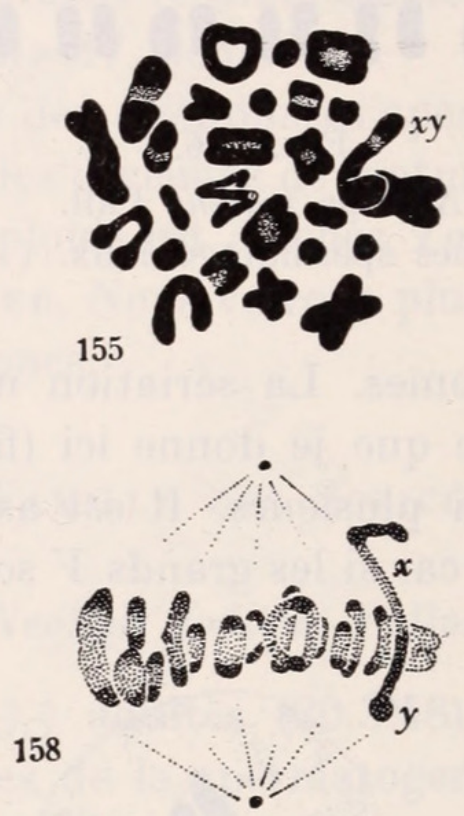

FIG. 154 à 159.

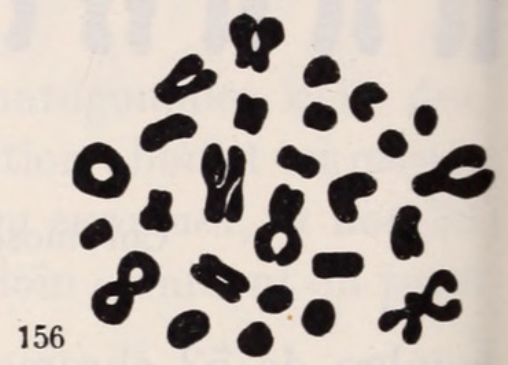

159

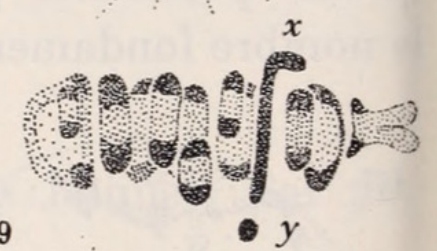

Neomys fodiens Pall.

Métaphases auxocytaires en vue polaire (154-156) et de profil (157-159). Hématoxyline (154-158), trichromique de Flemming (159). ( $\times$ 4000.)

vésiculaire comme chez les Chiroptères, et apparaît le plus souvent sous l'aspect d'un filament tantôt condensé et épais, tantôt allongé et fin. Sa forme est variable: anneau (fig. 147), figure en 8 (fig. 140, 151 et 152) sont les aspects les plus caractéristiques et les plus fréquents. Cette variabilité de forme rend très difficile l'interprétation du complexe $X Y$ qui constitue l'hétérocaryosome, car un schéma peut expliquer quelques figures observées, mais jamais toutes. D'autre part, on se heurte à un obstacle de nature physique: le pouvoir séparateur de l'objectif $(0,2$ micron) n'est pas suffisant pour permettre l'analyse précise d'éléments mesurant souvent moins de 0,3 micron d'épaisseur.

Les bivalents sont au nombre de 26 dans les métaphases de 
première dịvision de maturation (fig. 154-156). Disposés régulièrement en une plaque équatoriale petite, ils montrent les formes caractéristiques des éléments métacentriques: anneaux doubles ou triples, boucles verticales. Les plus petits sont punctiformes.

En profil, les hétérochromosomes sont facilement décelés (fig. 157-159). Leur structure rappelle celle que j'ai décrite chez Talpa: chromosome $X$ à bras inégaux, dans le rapport 3: 1, chromosome $Y$ punctiforme, placé à l'extrémité du bras long d' $X$. La figure 157 montre les deux chromosomes sexuels réunis: l'aspect sphérique de l' $Y$ n'apparait pas encore, et la tension anaphasique se manifeste par un léger étranglement dans la zone de liaison, située au niveau du plan équatorial. La séparation est plus avancée à la figure 158, et une strie plus sombre s'observe entre $X$ et $Y$. Enfin, à la figure 159, la disjonction est effective, et l'on peut alors juger des dimensions réelles des hétérochromosomes. La préréduction est constante.

Je n'ai pas observé les secondes cinèses de maturation.

\section{Crocidura russula Herm.}

M a té ri e l: 10 ô ô adultes, fixés en janvier, mars, avril, septembre, novembre.

O b s e r vations: Les divisions spermatogoniales, présentes toute l'année, ne sont jamais très abondantes, et leur fixation est rarement satisfaisante. Dans les figures les plus claires, j'ai cependant pu aisément compter 42 chromosomes (fig. 160 et 161), parmi lesquels on distingue un grand élément métacentrique, à bras inégaux, qui n'est autre que le chromosome $X$, et trois paires d'autosomes en forme de $V$. Les autres autosomes sont tous acrocentriques. Quant à l' $Y$, c'est probablement un petit $V$. Le nombre fondamental peut être fixé à 50 environ.

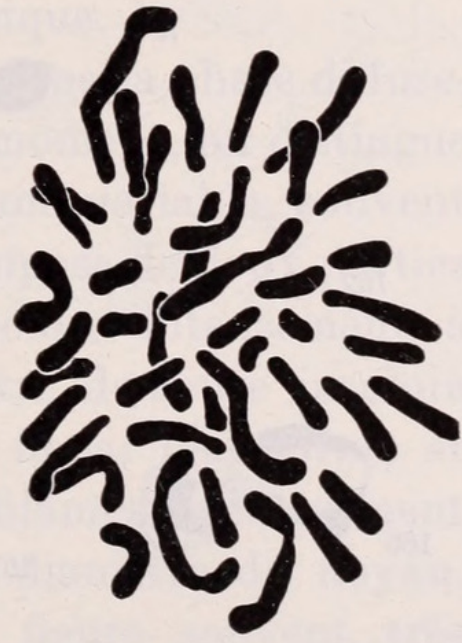

FIG. 160.

Crocidura russula Herm.

Métaphase spermatogoniale. Hématoxyline.

$(\times 4000$.) 
Ce matériel s'est révélé très favorable à l'étude des stades préméiotiques. L'intérêt qu'il y avait à les comparer à ceux de Sorex araneus m'a conduit à consacrer beaucoup de temps à leur analyse.

\section{$16 x\|3 x\|\|\|\|\|\|\|\|\|$ 2 \|\|\|\|\|\|$\cdots \|]$ !}

FIg. 161.

Crocidura russula Herm.

Chromosomes spermatogoniaux. $(\times 4000$. $)$

Je me suis toujours astreint à contrôler sur des coupes au Feulgen toutes les observations faites sur du matériel coloré à l'hématoxyline ou au violet cristal.
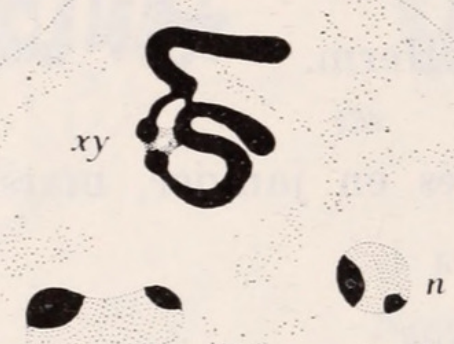

$n$

162

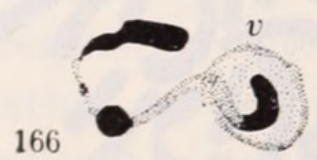

- ${ }^{n}$

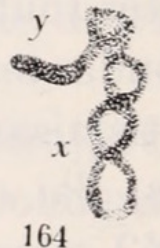

168

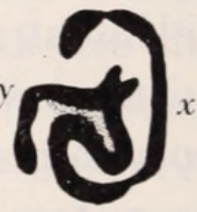

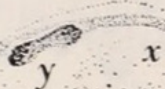

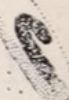

163

165

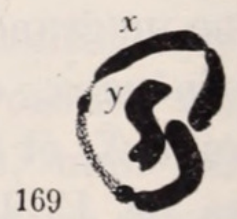

FIG. 162 à 169.

Crocidura russula Herm.

Fig 162 et 165 . Noyaux d'auxocytes au stade pachytène (diffus). $x, y=$ hétérochromosomes; $n=$ nucléole. - Fig. 163, 164, 166 à 169. Aspects divers du couple XY au stade pachytène. $v=$ vésicule hétérocaryotique. Hématoxyline $(162,164,166-169)$, fuchsine de Feulgen $(163,165) .(\times 4000$. 
Le ptoténie et zy goténie: Aucune structure hétérochromatique n'apparaît à l'intérieur du noyau. Les hétérochromosomes ne sont pas encore reconnaissables, et je n'ai pas vu la vésicule décrite à ce stade chez le Rat, par Minouchi (1928). Un ou deux petits nucléoles apparaissent dans le matériel coloré à l'hématoxyline. L'observation est d'ailleurs difficile, en raison de la petitesse du noyau et de l'abondance du chondriome.

$\mathrm{P}$ a c h y t é n i e : Ce stade peut être subdivisé en deux phases:

1. Une phase condensée, avec des chromosomes bien individualisés, fortement colorés, épais.

2. Une phase diffuse, reliant la phase précédente à la diploténie, et pendant laquelle les chromosomes sont très flous et peu colorés, parfois même invisibles dans les préparations à l'hématoxyline. Bien que la sériation de ces stades soit difficile à établir de façon certaine, je pense que cette phase fait suite à la pachyténie proprement dite, et dure jusqu'à la diploténie. On trouve en effet tous les degrés de transition entre la phase diffuse et la diploténie, d'une part, et entre ce dernier stade et la diacinèse d'autre part. En outre la structure des chromosomes à la phase diffuse, bien que très peu apparente, n'est jamais celle que l'on devrait observer si, comme le pense WiLson (1925) cette phase était intermédiaire entre diploténie et diacinèse: les filaments sont d'abord simples, et les boucles chiasmatiques n'apparaissent qu'à la fin, au moment où le noyau s'achemine vers la diploténie typique.

C'est au stade pachytène, et en particulier dans sa phase diffuse, que se révèlent les hétérochromosomes. A ce moment, on distingue dans le noyau deux ou trois nucléoles de forme variable, souvent sphériques ou ovoïdes. Chaque nucléole se compose de deux parties distinctes: l'une, fortement basophile, se colore intensément à l'hématoxyline et au violet, tandis que l'autre demeure incolore (fig. 162). Les autosomes sont très pâles et flous: leur forme et leur nombre échappent à l'analyse. Enfin, un filament relativement long, qui, étendu, serait aussi grand que le diamètre du noyau, dessine contre la membrane nucléaire une figure souvent très complexe et polymorphe. Dans les cas les plus simples (fig. 163, $165,167,168)$ on distingue deux parties d'inégale longueur: l'une relativement courte et intensément colorée est coudée presque à angle droit vers son milieu; l'autre, trois ou quatre fois plus longue, 
a la forme d'un cordon sinueux, souvent replié ou pelotonné (fig. 164). Ces deux parties sont reliées par leurs extrémités (fig. 163, 166, 167) ou accolées parallèlement sur une certaine longueur (fig. 165 et 168). Les liaisons paraissent de nature chiasmatique. Dans quelques cas (fig. 166), une partie du filament est engagée dans une vésicule pâle, qui n'est autre que l'hétérocaryosome. D'ailleurs de nombreux noyaux ne montrent au stade pachytène qu'une masse hétérochromatique opaque, à l'intérieur de laquelle
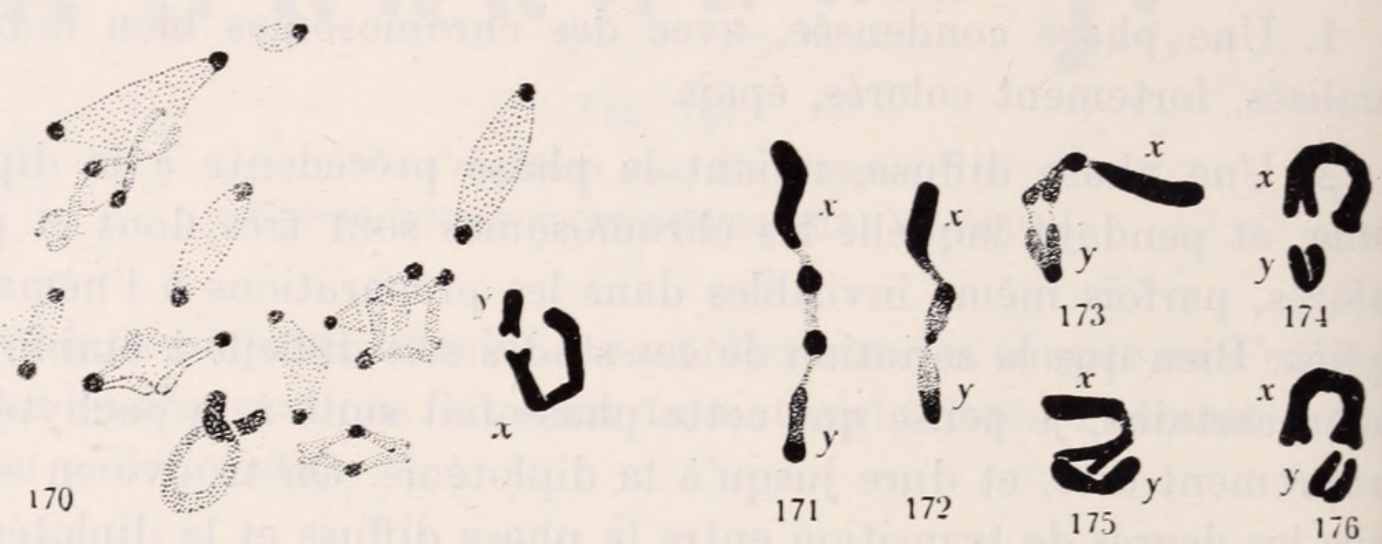

FIG. 170 à 176.

Crocidura russula Herm.

FIG. 170. Fin de diacinèse, avec hétérochromosomes bien visibles. FIg. 171 à 176. Aspect du couple XY à la diacinèse.

Hématoxyline. $(\times 4000$. $)$

on ne peut rien distinguer. Bien que la structure du filament hétérochromatique, très complexe, reste souvent inexplicable, il est clair que le long filament est le chromosome $X$, tandis que le plus petit représente son partenaire $Y$.

Diploténie: Ce stade peut rarement être observé, et paraît très fugace. Les hétérochromosomes ne se distinguent pas des autosomes.

D i a c i n è s e : En général, les chromosomes sexuels ne sont pas hétérochromatiques à ce stade. Toutefois, j'ai trouvé quelques images où ils étaient mis en évidence par une forte coloration différentielle (fig. 170-176). Beaucoup plus condensés qu'à la pachyténie, ils sont placés bout à bout, et montrent à leur point de contact une sorte de bifurcation indiquant vraisemblablement la présence de chiasmas terminalisés (fig. 173) ou déjà résolus (fig. 170, 174, 176). Dans quelques cas, des zones hétérochromatiques 
apparaissent encore: bras long de l' $X$, région centromérique, extrémité du bras court.

Première division de maturation (fig. 177179): La présence de vingt et un bivalents à la métaphase permet de confirmer le nombre diploïde 42. Le bivalent $X Y$ est en

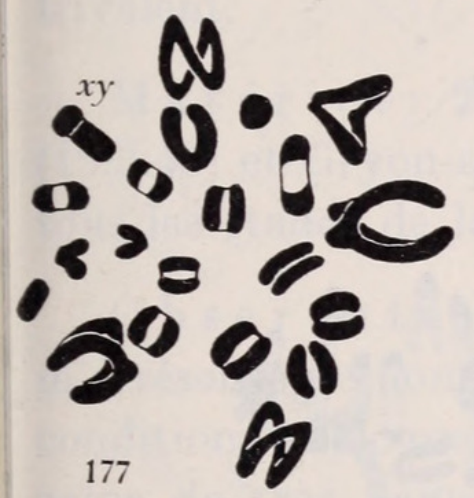

178
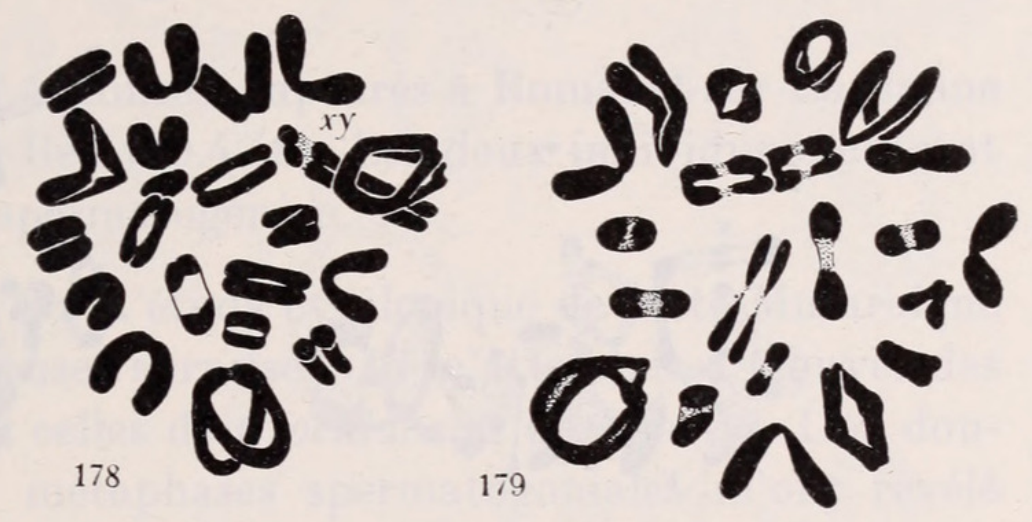

FIG. 177 à 179.

Crocidura russula Herm.

Métaphases auxocytaires, vue polaire.

Hématoxyline $(178,179)$, trichromique de Flemming (177). ( $\times 4000$.)

général facile à déceler, même en vue polaire (fig. 177 et 178). Sa position est presque toujours périphérique.

Les images de profil du même stade (fig. 180-183) permettent de suivre facilement l'évolution des chromosomes sexuels. L' $X$ apparaît comme un $V$ à bras inégaux, dont le plus court, relativement rigide, est associé à l' $Y$, tandis que le bras long, souvent replié et montrant une ou deux constrictions secondaires, est perpendiculaire au fuseau. Le chromosome $Y$ est massif, et prend tantôt la forme d'un bâtonnet, tantôt celle d'un petit $V$ à branches peu divergentes. Il est difficile de préciser si l'on a affaire à un chromosome acrocentrique dont les chromatides sont écartées, ou à un véritable métacentrique. Sa taille est approximativement égale à celle du bras court de l' $X$. On notera enfin un fait remarquable: alors qu'à la pachyténie et à la diacinèse, le bras non apparié de l' $X$ montrait souvent une forte hétérochromatie positive, la situation est inverse à l'anaphase de la première division: c'est alors le bras apparié qui se colore le plus, tandis que l'autre bras, dont les chromatides se séparent légèrement, reste pâle. La région centromérique est toujours intensément colorée.

Rev. Suisse de Zool., T. 56, 1949. 


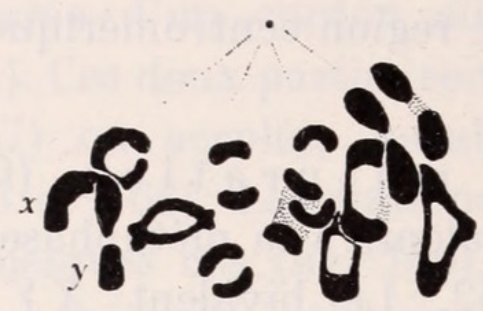

180

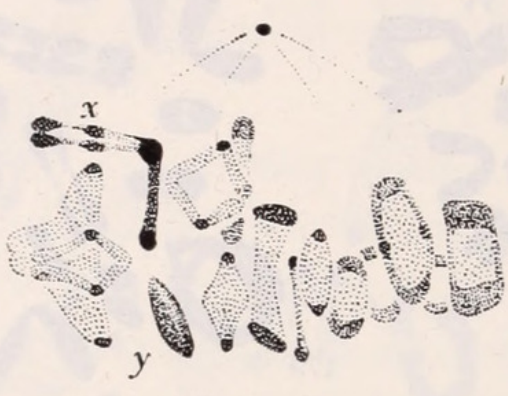

181

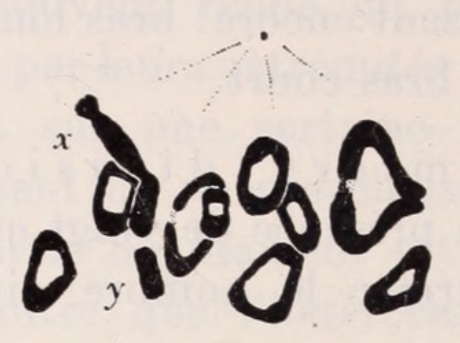

182
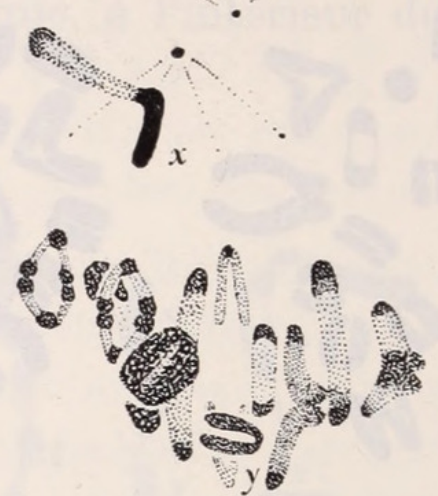

183

FIG. 180 à 183.

Crocidura russula Herm.

Métaphases auxocytaires; profils, avec paire XY.

Hématoxyline. $(\times$ 4000.)

Les bivalents autosomiques forment des $V$ à branches largement

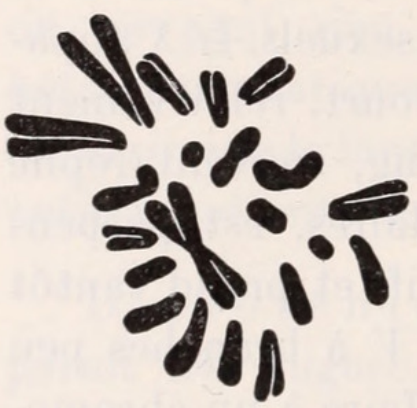

FIG. 184.

Crocidura russula Herm.

Seconde division de maturation. Hématoxyline. $(\times 4000$. divergentes, des anneaux parallèles ou perpendiculaires au plan équatorial.

A la télophase de la première division de maturation et durant l'intercinèse, les autosomes perdent leur colorabilité et deviennent invisibles, tandis que les hétérochromosomes retiennent énergiquement le colorant. Je n'ai jamais observé de cas certain de postréduction.

Seconde division de matu$\mathrm{rat}$ i on. Ces figures sont rarement très bien fixées. Les 21 dyades qui forment la plaque équatoriale (fig. 184) ont presque toutes la structure des éléments acrocentriques. 


\section{Sorex araneus $\mathrm{L}$.}

Je décrirai pour terminer le cas de cette Musaraigne, qui, seule parmi les Mammifères étudiés jusqu'à maintenant au point de vue cytologique, possède un dispositif hétérochromosomique de type trivalent.

M a t é r i e l: 2 ô ô adultes, capturés à Romanel-sur-Lausanne (15.3.48) et Gryon-sur-Bex (10.4.48). Les deux individus montrent tous les stades de la spermatogenèse.

O b s e r vatio n s: L'étude cytologique de cette Musaraigne m'a réservé de nombreuses surprises. Je m'attendais à trouver des conditions analogues à celles de Crocidura et de Neomys. Une douzaine de très bonnes métaphases spermatogoniales m'ont révélé d'emblée l'intérêt très particulier que présente, au point de vue cytologique, cette espèce si banale.

Sorex araneus possède 23 chromosomes dans ses cellules diploïdes (fig. 185-192). La fixation, généralement excellente en raison du nombre très bas des chromosomes, conserve bien leur structure fine, et permet de connaître avec beaucoup de précision la position du centromère, dont l'emplacement est marqué par une zone achromatique plus ou moins étendue. Ce dernier fait pourrait d'ailleurs être une cause d'erreur lors des numérations, car on peut facilement prendre un chromosome en $V$ pour deux éléments acrocentriques rapprochés fortuitement à leur apex. Cependant, la comparaison de plusieurs figures permet de lever facilement ce doute.

J'ai établi avec beaucoup de soin les caryogrammes (fig. 193) correspondant aux huit métaphases spermatogoniales reproduites ici (fig. 185-192), pensant mettre en évidence une digamétie de type $X O$, dont l'éventualité me paraissait découler tout naturellement du nombre impair de chromosomes. Je n'ignore pas les critiques que suscite cette méthode. Justifiées lorsque les chromosomes sont nombreux et peu différents de taille, elles sont sans objet lorsque, comme chez Sorex, chaque chromosome a son aspect particulier, et que les tailles des différents éléments sont peu comparables. Or, toutes les tentatives d'arrangement m'amenèrent au même résultat; je pouvais former dix paires autosomiques, toutes 

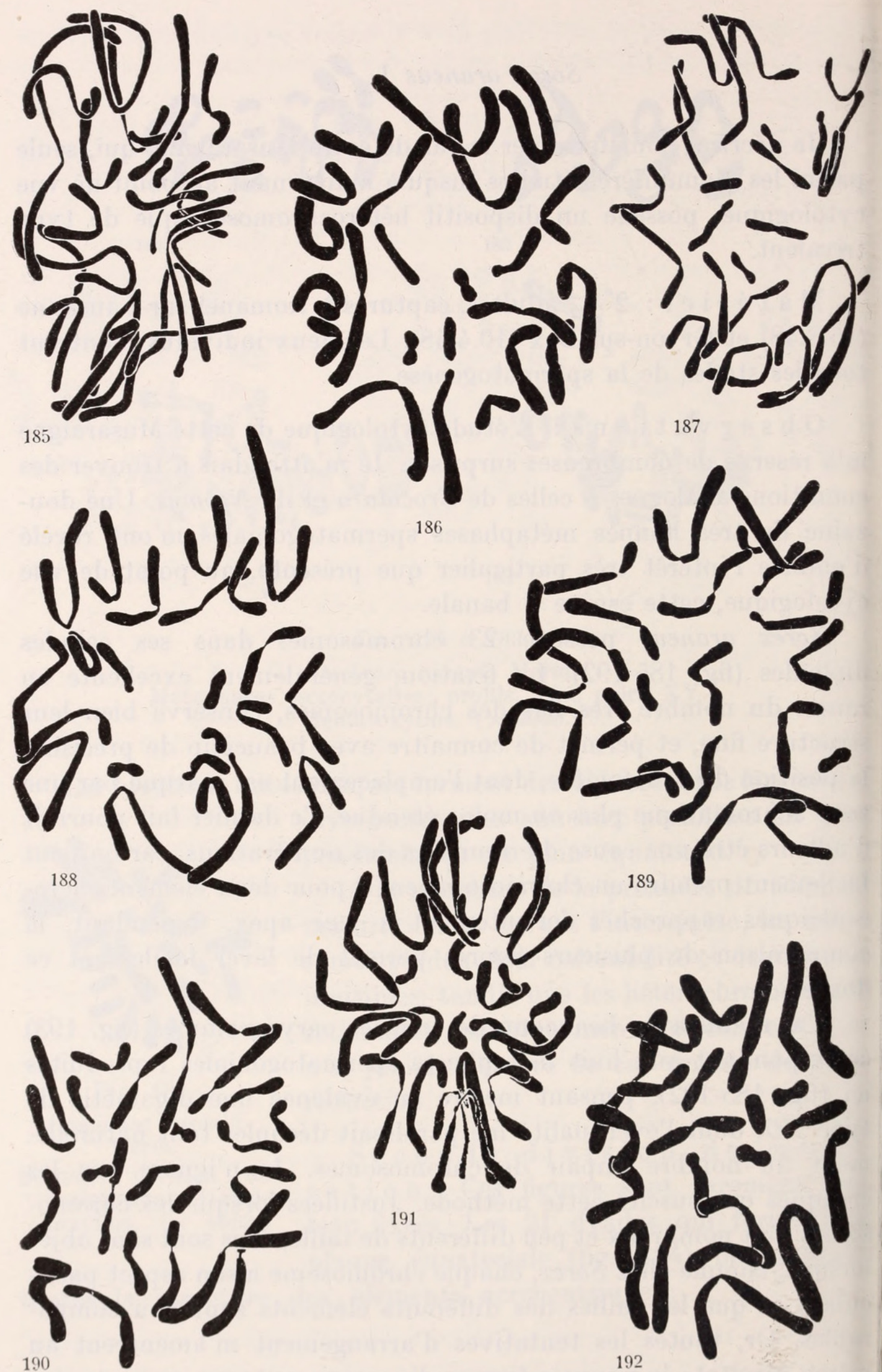

FIG. 185 à 192.

Sorex araneus $\mathrm{L}$. 
constituées d'éléments métacentriques, mais il restait toujours trois chromosomes qui ne pouvaient être appariés: un grand élement acro- ou télocentrique, un métacentrique de taille moyenne, et un bâtonnet court et rigide, dont le mode d'attachement n'est pas clairement établi.

Obligé par conséquent de rejeter l'idée d'une digamétie $X O$, j'envisageai alors deux possibilités: digamétie $X Y$ et existence d'un univalent, ou formation à la méiose d'un trivalent composé des trois éléments non appariés dans les caryogrammes. L'étude des divisions de maturation a justifié la seconde hypothèse, mais avant d'en parler, je décrirai d'abord les stades pré-méiotiques. Leur étude se heurte aux mêmes difficultés que chez Crocidura et Neomys, aussi ne donnerai-je que les observations qui m'ont paru tout à fait certaines.

L e p t o té nie: Les préparations au Feulgen ne montrent en général qu'un réseau de filaments très fins. Dans quelques cas cependant, on remarque dans le noyau une masse plus colorée, appliquée contre la membrane nucléaire. Cette sphérule est très petite, et son diamètre est à peu près égal à l'épaisseur des chromosomes pachytènes. Dans les préparations colorées à l'hématoxyline, les chromosomes sont très fins aussi, mais un filament plus épais et plus coloré, souvent renflé en massue à l'une de ses extrémités, apparaît parmi eux. D'autres noyaux montrent plutôt une vésicule analogue à celle que l'on peut observer dans les noyaux colorés au Feulgen. Je pense que vésicule et filament hétérochromatique ne représentent que deux aspects d'une même réalité, à savoir une partie des hétérochromosomes. L'aspect vésiculaire provient peutêtre de la fixation, lorsque celle-ci respecte la substance hétérocaryosomique.

Z y g o t é n i e : Il n’y a rien de spécial à signaler: la vésicule est toujours visible dans les noyaux, mais on ne voit plus de filament hétérochromatique.

P a c hyténie: C'est le stade le plus important, car il permet d'expliquer l'origine du trivalent, comme nous le verrons plus tard. Désireux d'éviter des erreurs, je n'ai travaillé qu'avec des coupes colorées à la fuchsine de Feulgen. Les autosomes se colorent bien, du moins dans la phase "condensée ", et leur contour 
a l'aspect floconneux caractéristique de ce stade. On distingue parmi eux, appliquée contre la membrane nucléaire, la vésicule hétérochromatique déjà observée au stade leptotène (fig. 194). Colorée en rouge pâle, elle ne laisse en général pas apparaître son contenu. Mais ici intervient un fait absolument nouveau: cette vésicule est

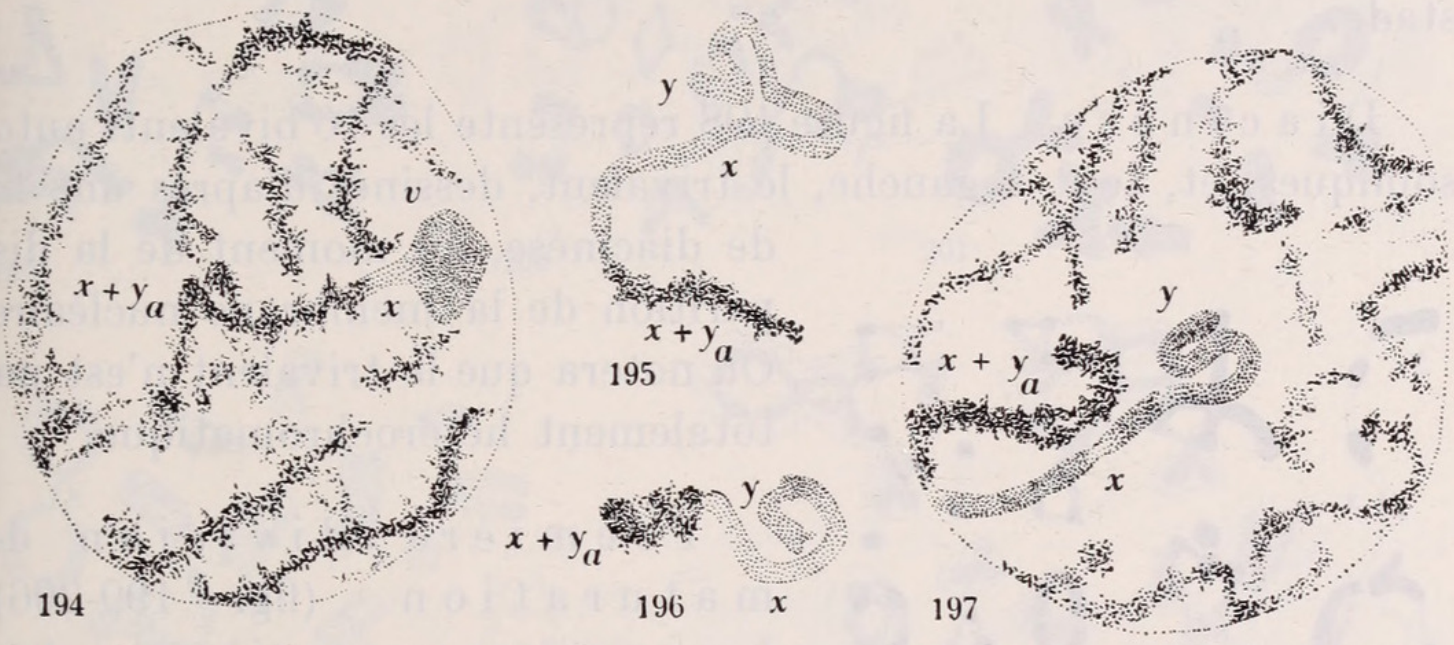

FIG. 194 à 197.

Sorex araneus L.

Fig. 194 et 197. Deux noyaux d'auxocytes au stade pachytène, avec le filament hétérochromosomique; $\vartheta=$ vésicule hétérocaryotique. - FIg. 195 et 196. Filament hétérochromosomique, stade pachytène. $x, y, y a$ : composants du trivalent. Fuchsine de Feulgen. ( $\times$ 4000.)

reliée par un fin connectif à un fragment chromosomique, dont l'aspect irrégulier èt plumeux est typiquement autosomique. Dans d'autres noyaux (fig. 195-197), la vésicule a disparu, libérant un filament hétérochromatique un peu plus clair que les autosomes, à bords nets et d'épaisseur constante, dont la structure rappelle beaucoup celle $\mathrm{du}$ filament hétérochromosomique de Crocidura russula (cf. fig. 163, 166). Il est toujours relié à un segment autosomique. L'ensemble des deux tronçons mesure environ vingt microns: la partie hétérochromatique a une longueur de douze microns, tandis que la partie autosomique mesure huit microns. Lorsque la vésicule a disparu (fig. 195-197), le filament hétérochromatique garde un aspect pelotonné, comme chez Crocidura russula.

A la phase diffuse de la pachyténie, on ne remarque rien de nouveau en ce qui concerne la partie hétérochromatique de ce qu'on peut déjà appeler le trivalent, mais le fragment autosomique suit en revanche l'évolution normale d'un autosome et s'estompe, 
si bien qu'à ce stade, l'aspect est à peu près celui d'un cordon vermiforme plus ou moins enroulé en spirale, ou d'une vésicule appliquée contre la membrane du noyau. Toutefois, on aperçoit encore, bien que difficilement, la liaison avec l'autosome.

D i ploténie: Je n'ai pas d'observations relatives à ce stade.

D i a c i n è s e : La figure 198 représente les 10 bivalents autosomiques, et, tout à gauche, le trivalent, dessinés d'après une fin de diacinèse, au moment de la dis-

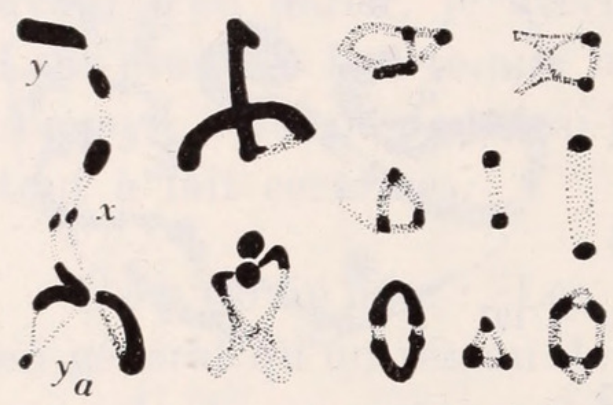

FIG. 198.

Sorex araneus L.

L'ensemble des bivalents, à la fin de la diacinèse. $x-y-y a=$ trivalent sexuel Hématoxyline. $(\times$ 4000. $)$ parition de la membrane nucléaire. On notera que le trivalent n'est pas totalement hétérochromatique.

Première division de maturation (fig. 199-206): Les premières numérations me donnèrent l'impression qu'il $\mathrm{y}$ avait 12 bivalents. Mais je remarquai bientôt que l'un des plus petits éléments était toujours placé à l'extrémité d'un bivalent qui paraissait très dyssymétrique (fig. 199 et 206). Une telle position si constante ne peut être due au hasard: les figures de profil donnent d'un coup la clé du double problème posé par les caryogrammes et par les cinèses auxocytaires. Elles montrent en effet sans aucun doute l'existence, chez Sorex araneus, d'un trivalent formé d'un chromosome en $V$ associé par ses deux bras avec deux éléments acro- ou télocentriques (fig. 207-212). Le point d'attachement de l'élément métacentrique central est marqué par une coudure nette. De part et d'autre, les deux bras de ce chromosome, que je désigne par la lettre $X$, sont inégaux. Le plus long s'apparie avec un élément acrocentrique ou peut-être télocentrique, que je nomme $Y a$, et dont la longueur est légèrement inférieure à celle du bras long de l' $X$. Un ou deux chiasmas se forment entre le bras long de l' $X$ et son homologue $Y a$, et dans de nombreux cas, les extrémités distales des chromatides, encore appariées, forment deux masses opaques, tandis que leurs portions proximales, séparées, restent presque invi- 
sibles (fig. 209, 210). Le bras court de l' $X$ est en relation avec un petit chromosome, que je nomme $Y$; c'est le plus petit des trois éléments non appariés dans les caryogrammes. Massif et rigide, il
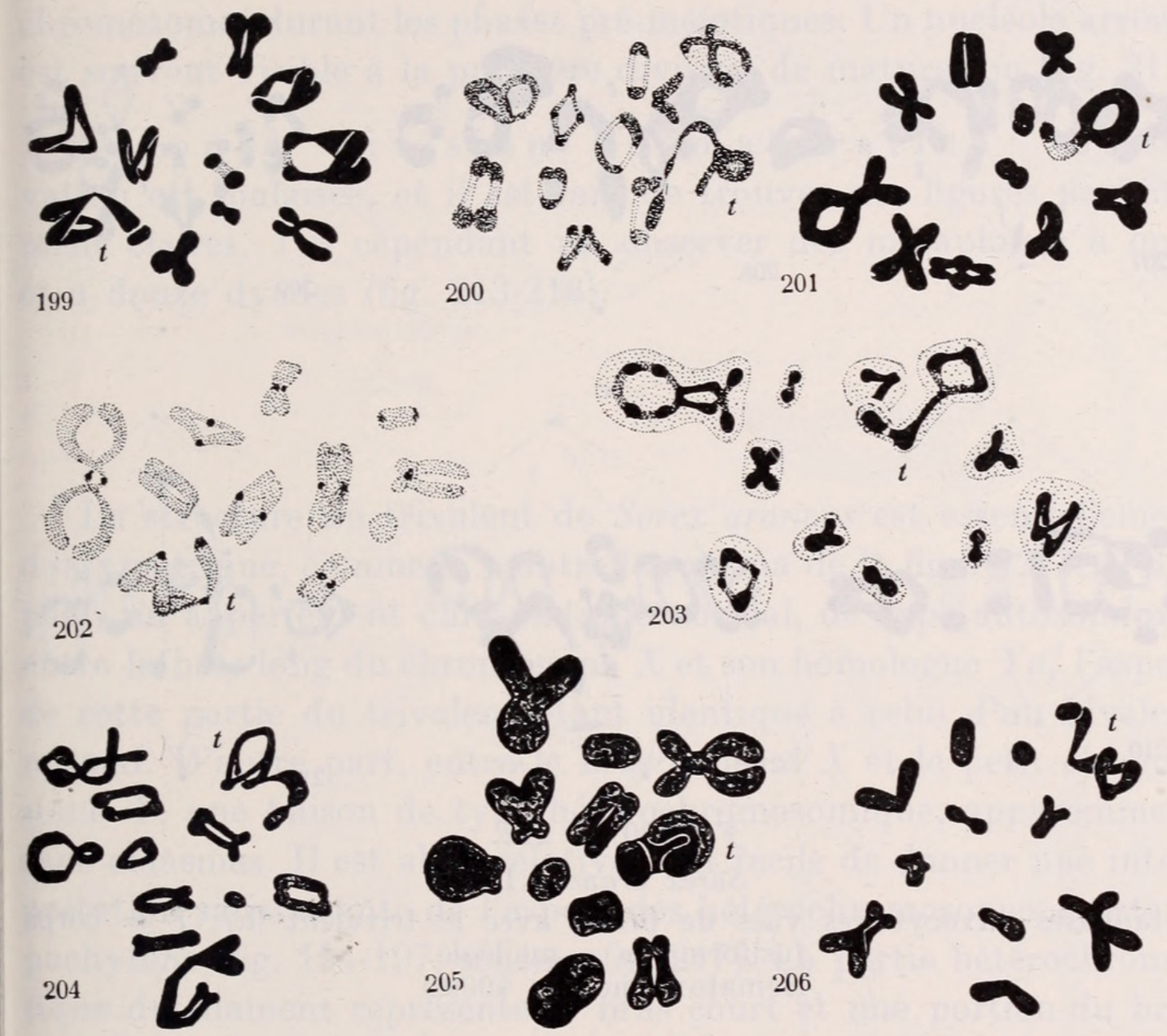

FIG. 199 à 206.

Sorex araneus L.

Métaphases auxocytaires en vue polaire (fig. 200 oblique). $t=$ trivalent sexuel. Hématoxyline $(199-204,206)$ et fuchsine de Feulgen $(205)$. ( $\times 4000$.)

est presque toujours placé dans le prolongement du bras court d' $X$, à une distance d'environ 0,5 micron. Dans quelques cas, j'ai remarqué entre $X$ et $Y$ un fin connectif (fig. 212). Le chromosome $X$ est toujours orienté vers l'un des pôles, $Y$ et $Y a$ vers l'autre, et la coorientation se produit, comme pour les autosomes, au moment de la mise au fuseau. Dans quelques cas, une partie du bras court de l' $X$ est hétérochromatique négative (fig. 209), mais l'extrémité du segment apparié avec $Y$ reste toujours colorée aussi intensément que celui-ci. 
Les dix bivalents autosomiques sont normaux, mais parfois montrent une coloration chromonématique très curieuse, la matrix

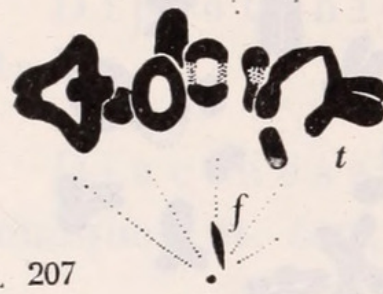

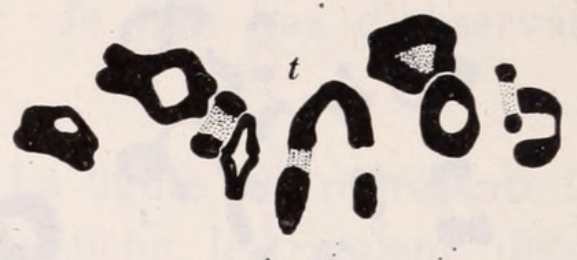

208

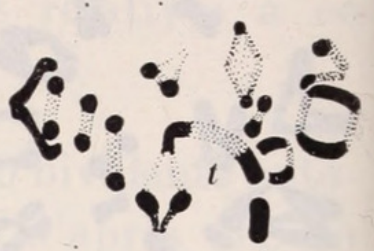

209

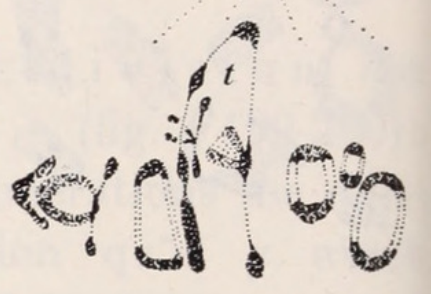

212
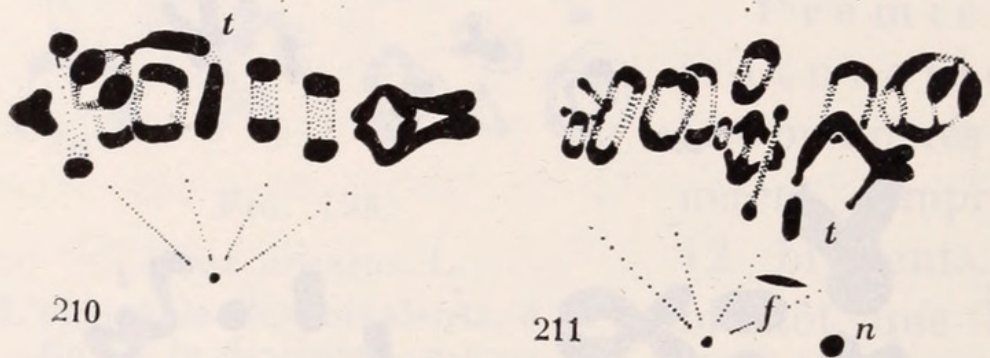

FIG. 207 à 212.

Sorex araneus L.

Métaphases auxocytaires vues de profil, avec le trivalent $(t.) ; f=\operatorname{corps}$ fusiforme, $n=$ nucléole.

Hématoxyline. $(\times 4000$.

des chromatides restant presque incolore (fig. 203). Avec la fuchsine de Feulgen, au contraire, la matrix, très épaisse et gonflée, se colore intensément, et donne l'aspect empâté de la figure 205.
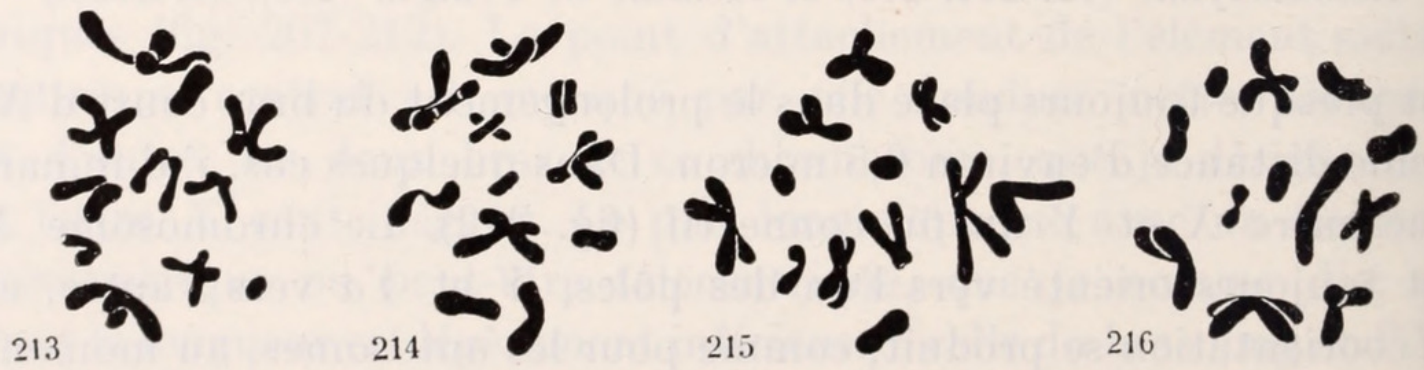

FIG. 213 à 216.

Sorex araneus $\mathrm{L}$.

Métaphases de seconde division de maturation.

FIG. 213 et 214. 11 dyades. - Fig. 215 et 216. 12 dyades.

Hématoxyline. $(\times 4000$. $)$ 
On remarque dans presque toutes les cellules, durant les stades de l'accroissement et pendant la première division de maturation, un corpuscule fusiforme, très mince et long d'un 'micron environ. Il se colore à l'hématoxyline, et évolue à proximité des hétérochromosomes durant les phases pré-méiotiques. Un nucléole arrondi est souvent visible à la première division de maturation (fig. 211).

Seconde division de maturation: L'observation est malaisée, et il est rare de trouver des figures parfaitement claires. J'ai cependant pu observer des métaphases à onze et à douze dyades (fig. 213-216).

La structure du trivalent de Sorex araneus est essentiellement dyssymétrique, comme le montre le schéma de la figure 217. D'une part, un appariement chiasmatique normal, de type autosomique, entre le bras long du chromosome $X$ et son homologue $Y a$, l'aspect de cette partie du trivalent étant identique à celui d'un bivalent normal. D'autre part, entre le bras court d' $X$ et le petit chromosome $Y$, une liaison de type hétérochromosomique, apparemment sans chiasmas. Il est alors relativement facile de donner une interprétation satisfaisante de l'aspect des hétérochromosomes au stade pachytène (fig. 194-197, schéma fig. 217). La partie hétérochromatique du filament représente le bras court et une portion du bras long de l' $X$, et le chromosome $Y$, qui est en général en continuité avec l'extrémité du bras court d' $X$. Ces deux composants du trivalent - partie hétérochromatique d' $X$, et $Y$ - évoluent partiellement dans une vésicule hétérochromatique (hétérocaryosome), semblable à celle que j'ai décrite chez les Chiroptères et chez quelques Insectivores. La partie autosomique se compose de l'extrémité du bras long de l' $X$, associée en parasynapsis normale avec le chromosome $Y a$ qui lui est homologue.

Il est difficile de se faire une idée exacte de la nature des liaisons entre $X$ et $Y$. A la métaphase, les deux chromosomes sont presque toujours déjà séparés. C'est done plus tôt qu'il faudrait observer leur disposition; malheureusement, les figures diacinétiques sont très rares; celle que j'ai donnée (fig. 198) montre bien l' $Y$, reconnaissable à son aspect rigide et hétérochromatique, relié sans 
apparence de chiasmas à l'extrémité du bras court de l' $X$. Si nous cherchons plus tôt encore, au stade pachytène par exemple, nous retrouvons la même disposition relative des deux éléments (fig. 196,
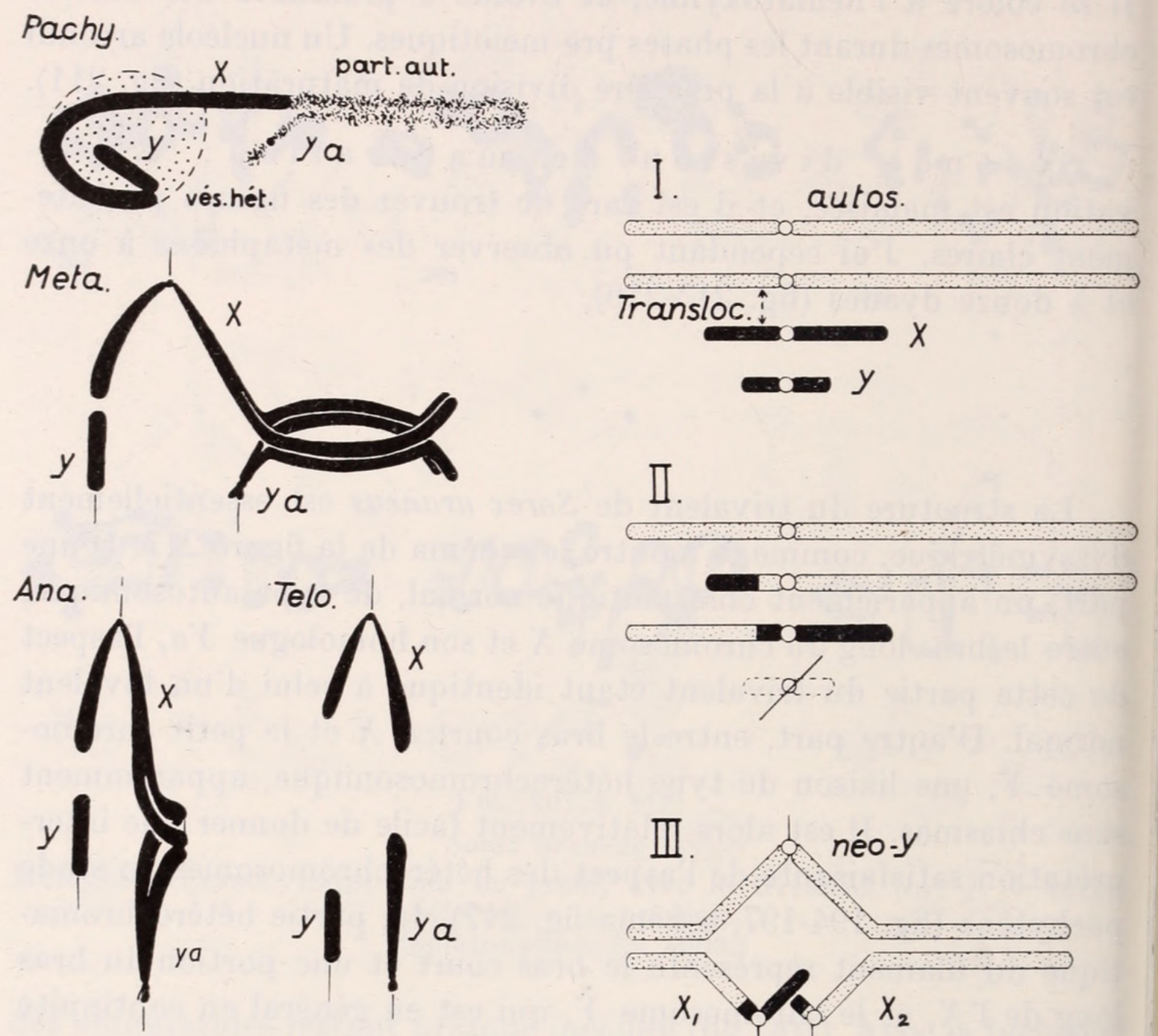

FIG. 217.

Sorex araneus L.

Schéma montrant l'évolution du trivalent à la première division de maturation. Pachy.$=$ stade pachytène. Méta. $=$ métaphase. Ana. $=$ anaphase. Télo. = télophase. Vés ${ }^{\cdot}$ hét. $=$ vésicule hétérocaryotique. Part. aut.= partie autosomique du chromosome $X$.

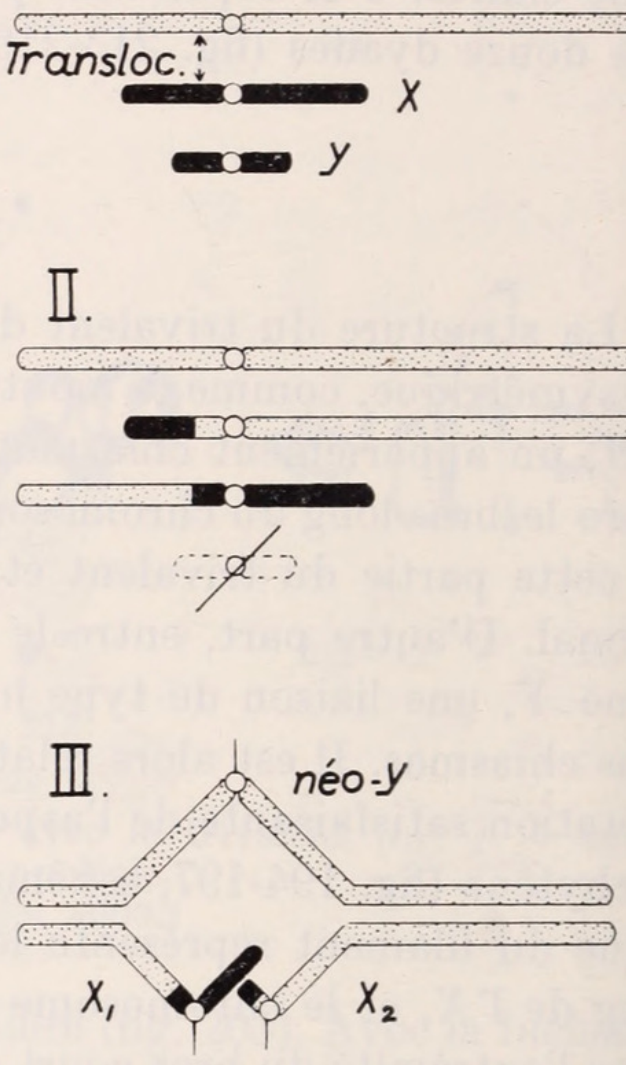

FIG. 218.

Schéma illustrant la première hypothèse sur l'origine du trivalent sexuel de Sorex araneus. Autos. = paire d'autosomes. Transloc. $=$ translocation, indiquée par les flèches. (Cf. page 447.)

197; schéma fig. 217). $X$ et $Y$ sont placés bout à bout, en continuité, ou parfois parallèlement, mais sans apparence très nette d'une liaison chiasmatique. Il n'est pas impossible que d'autres phéno- 
mènes physiques entrent en jeu: attraction entre segments pairs ou entre télomères, par exemple.

On peut émettre de nombreuses hypothèses pour expliquer la genèse du trivalent de Sorex araneus. J'en ai retenu deux, la seconde me paraissant de beaucoup la plus probable.

P r e mi ère h y p o th è s e: Nous partons d'une espèce à digamétie $X O$, ou nous supposons la perte de l' $Y$ (schéma fig. 218). Une translocation réciproque entre l'ancien $X$ et l'un des éléments d'une paire d'autosomes en $V$ donne, avec l'autre élément de la paire d'autosomes, resté intact, trois chromosomes homologues qui s'associent à la méiose, par leurs segments correspondants. Les deux chromosomes ayant subi la translocation portent chacun une partie de l'ancien $X$, et doivent done se nommer $X_{1}$ et $X_{2}$. L'autosome qui leur est homologue devient un néo- $Y$. Il est évident que la nomenclature que j'ai adoptée plus haut n'est pas adaptée à ce schéma.

C r it i q u e : Cette hypothèse a contre elle deux faits importants. Tout d'abord, elle suppose à l'origine une espèce à digamétie $X O$, alors que toutes les Musaraignes étudiées jusqu'à maintenant relèvent du schéma $X Y$; quant à la disparition de l' $Y$, c'est une supposition toute gratuite. En second lieu, elle ne tient pas compte de la structure dyssymétrique du trivalent au stade pachytène, où l'une des extrémités est de nature autosomique, l'autre de nature hétérochromosomique. Or, le schéma que suggère la première hypothèse est symétrique, sinon quant à la forme, du moins quant à la nature des segments situés de part et d'autre du centromère du néo- $Y$.

S e c o n d e hy p ot h ès e: Nous supposons à l'origine une espèce à digamétie $X Y$. Le chromosome $Y$ reste inchangé, et $X$ est un élément métacentrique. Une translocation soude, sur l'extrémité du bras non apparié de l' $X$ (segment différentiel) un fragment autosomique relativement long, provenant d'un chromosome acroou télocentrique (schéma fig. 219 I et II.). Le nouvel $X$, ainsi prolongé, est homologue à l' $Y$ par son bras court, et à un autosome $Y a$ par son segment autosomique. Il s'appariera donc avec ces deux chromosomes à la méiose, formant ainsi le trivalent. (Schéma fig. 219, III.) 
Critique: Cette hypothèse a l'avantage de sa simplicité, et de son point de départ conforme au schéma général de digamétie $X Y$, normal chez les Mammifères. Elle explique remar-
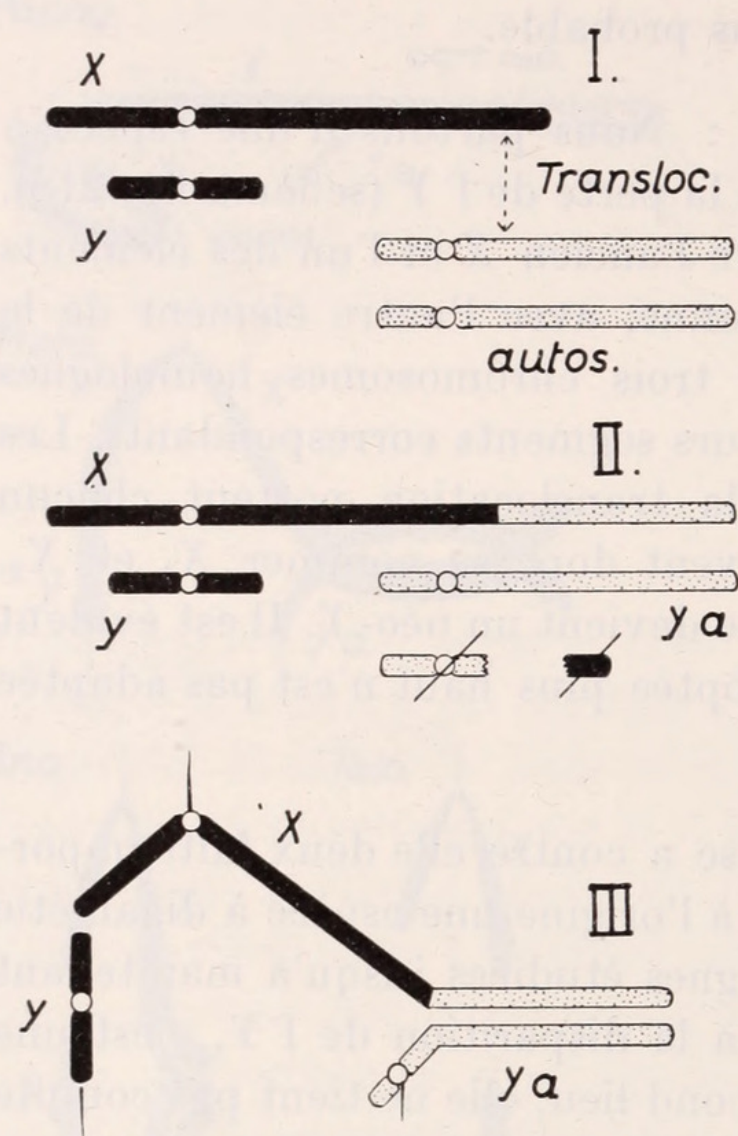

FIG. 219.

Schéma illustrant la seconde hypothùss sur l'origine du trivalent de Sorex araneus. (Cf. page 447.) quablement bien l'aspect des figures observées au stade pachytène: toute la partie hétérochromatique du trivalent (fig. 197 et schéma fig. 217), si semblable à l'XY pré-méiotique de Crocidura russula (fig. 163, 166) représente le matériel provenant de l'ancien $X Y$ de l'espèce primitive; le point de transition marque l'endroit où s'est produite la translocation, et le fragment autosomique, d'aspect plumeux et irrégulier, n'est autre que le segment d'autosome soudé à l' $X$, et apparié à son homologue $Y a$.

Formule de la fem ell e : Si la première hypothèse est fondée, la femelle doit normalement posséder $24 \mathrm{chro-}$ mosomes, soit 20 autosomes $+2 X_{1}+2 X_{2}$ puisque les gènes déterminant le sexe sont

répartis sur les chromosomes $X_{1}$ et $X_{2}$ (nomenclature du schéma fig. 218). Si c'est la seconde hypothèse qui est exacte, on peut s'attendre à trouver chez la femelles 22 chromosomes, soit 20 autosomes $+2 X$. J'espère pouvoir me procurer prochainement des femelles, dont l'analyse autorisera une conclusion définitive.

Le cas de Sorex présente un intérêt multiple:

Tout d'abord, c'est parmi tous les Euthériens l'espèce qui possède, de façon certaine, le nombre de chromosomes le plus bas. Il est vrai que Pontecorvo (1943) signale chez Cricetulus griseus un nombre encore plus petit ( $2 N=14)$, mais la qualité très médiocre 
du matériel, fixé au Bouin et conservé pendant seize ans dans l'alcool, n'inspire pas entièrement confiance, et les résultats de Pontecorvo devront encore être confirmés. Le Mammifère euthérien qui, après Sorex araneus, possède le nombre le plus bas de chromosomes, est un Rongeur étudié par OGuma (1937), Microtus montebelli $(2 N=31)$. De nombreux Marsupiaux présentent des formules diploïdes comprises entre douze et vingt-deux.

Il est intéressant de noter que chez Microtus montebelli, comme chez Sorex araneus, le nombre diploïde peu élevé va de pair avec une forte proportion de chromosomes en $V$. Si nous admettons que les grands $V$ proviennent de fusions centriques de chromosomes en $I$, et que celles-ci sont dues à des translocations réciproques, on peut supposer qu'un mécanisme évolutif, après avoir porté sur les autosomes, est également intervenu dans les modifications structurales des chromosomes sexuels. Microtus montebelli a seulement perdu son chromosome $Y$, tandis que chez Sorex, l' $X$ s'est combiné avec une paire d'autosomes, réalisant un type plus complexe d'hétérochromosomes. Ceci nous montre enfin que, outre les schémas classiques $X O$ et $X Y$, il existe encore d'autres possibilités de digamétie chez les Mammifères.

L'existence d'un trivalent n'a encore jamais été signalée chez un Vertébré. Chez les Invertébrés, en revanche, des hétérochromosomes multiples de ce type ont été décrits dans de nombreux cas, en particulier chez des Orthoptères, des Hémiptères, des Coléoptères et des Diptères. Enfin, chez un Kangourou, Macropus ualabatus, Agar (1923) a mis en évidence un quadrivalent formé par l'union d'une tétrade autosomique avec l'X $Y$. Matthey (1949 b) estime qu'AGAR a peut-être interprété à tort comme quadrivalent ce qui serait en réalité un trivalent analogue à celui de Sorex. Il serait heureux que l'étude de Macropus ualabatus soit reprise.

L'analyse détaillée du cas de Sorex araneus m'a montré combien il est difficile de résoudre la question des hétérochromosomes chez les Mammifères. Si le nombre de chromosomes n'avait pas été si bas, si les caryogrammes n'avaient été si clairs, grâce à l'excellente conservation des caractères morphologiques de chaque chromosome; si enfin, les divisions réductionnelles de profil n'avaient pas montré la structure de tous les bivalents, jamais je n'aurais pu résoudre ce problème et déceler le trivalent. Si un tel mécanisme existe chez d'autres Mammifères à nombre chromosomique plus. 
élevé, il est très probable qu'il pourra échapper à l'observateur le plus perspicace.

\section{DISCUSSION}

J'ai examiné deux espèces qui avaient déjà fait l'objet d'une étude antérieure. Mes résultats sont en accord avec ceux de Painter (1925) en ce qui concerne le Hérisson, et complètent les observations de ce cytologiste. Ils contredisent par contre ceux de Koller (1936), qui, avant moi, a étudié la spermatogenèse de la Taupe (Talpa europaea L.). En effet, Koller trouve chez cette espèce 38 chromosomes, alors que je n'en compte que 34 ; mais la discordance entre ces résultats ne se borne pas à la numération: alors que mes dessins montrent clairement que chez Talpa, tous les chromosomes, à l'exception de l' $Y$, sont métacentriques, Kolle R dessine plus de 10 éléments typiquement acrocentriques (fig. 10, p. 193). Mais la surprise augmente lorsqu'on compte les chromosomes dessinés dans cette figure: l'auteur affirme en avoir vu 38, mais il n'en a représenté que 34. Par contre la figure $1 a$, concerernant Putorius furo, espèce étudiée par Koller et décrite dans le même mémoire montre 38 chromosomes, alors que l'auteur donne le nombre diploïde 34 pour cette espèce. Les dessins sont probablement exacts, mais ils contredisent le texte. La situation est inversée à la figure 4, où les dessins $a$ et $b$, représentant une diacinèse de Putorius furo, indiquent la présence de 17 bivalents, tandis qu'on en compte 19 dans les dessins $c$ et $d$ relatifs à Talpa.

L'auteur dit avoir reçu son matériel, déjà inclu, d'un collègue de Londres. Il n'est pas impossible que les pièces aient été mélangées, ce qui pourrait expliquer les regrettables discordances numériques entre les dessins des différents stades et le texte. Dans ces conditions, on ne peut guère tenir compte des conclusions de Koller, concernant la structure des hétérochromosomes, ni de la figure 6 de son travail.

Les observations de TAteishi sur Crocidura murina L, très voisine de $C r$. russula au point de vue systématique, sont fort semblables à celles que j'ai pu faire chez cette dernière espèce.

Les nombres diploïdes et fondamentaux ne diffèrent que de deux et trois unités respectivement. Le chromosome $X$ est un très 
grand élément chez les deux espèces, l' $Y$ semble un peu plus grand chez Cr. murina. Enfin, Tateishi a fait les mêmes observations que moi en ce qui concerne l'hétérochromatie des chromosomes sexuels.

\section{Les hétérochromosomes des Insectivores.}

A part Sorex araneus, tous les Insectivores étudiés jusqu'à maintenant par Painter (1925), Koller (1936), Tateishi (1938) et moi-même, soit six espèces appartenant à trois familles différentes,

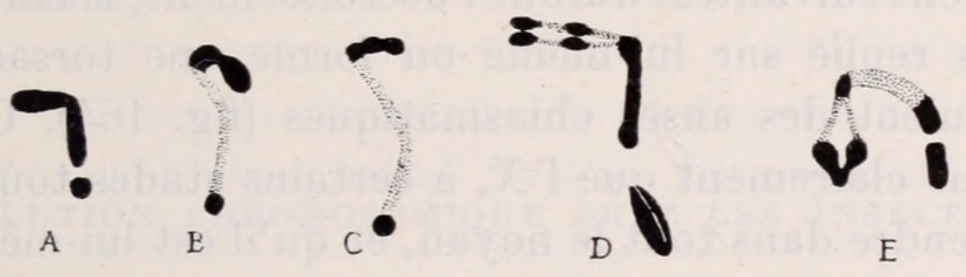

FIG. 220.

Aspect des hétérochromosomes à la métaphase auxocytaire, chez les Insectivores.

A : Erinaceus europaeus L. - B : Talpa europaea L. - C: Neomys fodiens Pall. - D : Crocidura russula Herm. - E: Sorex araneus L. (Trivalent.)

ont une digamétie de type $X Y$. Le chromosome $X$ est toujours métacentrique, et l' $Y$ en général petit, sphérique et punctiforme chez les Talpidae, chez Erinaceus et Neomys, est un peu plus grand et probablement métacentrique chez Crocidura. Dans l'ensemble, les hétérochromosomes des Insectivores sont semblables à ceux des Chiroptères (fig. 220).

L i a i s o n e n t r e $X$ e t $Y$. - Fidèle à l'hypothèse qu'il a le premier émise avec Darlington (1934), Koller admet chez Talpa, comme d'ailleurs chez tous les Euthériens à digamétie $X Y$, une liaison chiasmatique entre le chromosome $Y$ et le segment pair de l'X, localisé dans le bras court chez Talpa. Tateishi (1938) ne donne aucune indication à ce sujet pour les deux espèces étudiées par lui, mais ses figures d' $X Y$ de profil (fig. 1, p. 157) semblent montrer des liaisons chiasmatiques conformes à la conception de Koller et Darlington. L'auteur mentionne la formation d'anneaux dans les parties en contact de l' $X$ et de l' $Y$. J'ai fait des observations analogues chez $C r$. russula, et je pense qu'il s'agit d'un enroulement relationnel des chromatides, après rupture des

Rev. Suisse de Zool., T. 56, 1949. 
liaisons entre les hétérochromosomes. Quant à Painter, il n'a examiné que des divisions diploïdes.

Les observations que j'ai pu faire chez Talpa, Neomys et Crocidura s'accordent avec l'hypothèse de Koller et Darlington, mais le très grand polymorphisme des chromosomes sexuels durant les stades préméiotiques rend très difficile, pour ne pas dire impossible même, la réduction à un seul schéma d'une réalité si riche en diversité. On peut toutefois tenter de dégager quelques caractères généraux des hétérochromosomes; si l'on compare entre elles les figures 147, 149-152 (Neomys) et 163-169 (Crocidura), on arrive aux conclusions suivantes: durant l'accroissement, le chromosome $X$ est toujours replié sur lui-même ou forme une torsade dont les boucles simulent des anses chiasmatiques (fig. 164). Cette disposition indique clairement que l' $X$, à certains stades tout au moins, ne peut s'étendre dans tout le noyau, et qu'il est lui-même contenu dans une vésicule dont la nature reste inconnue: probablement une simple gouttelette de substance basophile, sans membrane propre. Cette vésicule disparaît plus ou moins tôt, ou tout au moins devient transparente et incolore, au cours de la pachyténie. De nombreuses figures montrent une parasynapsis entre $X$ et $Y$, mais il est rare de voir des chiasmas typiques.

A la diacinèse, les deux hétérochromosomes sont disposés bout à bout, et parfois déjà séparés. Enfin, lors de la métaphase de la première division de maturation, l' $Y$ est toujours placé dans le prolongement du bras court (Crocidura) ou long (Neomys, Talpa) de l' $X$.

\section{Pré-réduction ou post-réduction.}

Je n'ai observé chez les Insectivores que des figures de préréduction, et ce processus paraît être constant dans ce groupe, puisque Tateishi (1938) a fait les mêmes remarques. D'ailleurs, c'est le seul mécanisme qui soit compatible avec l'existence du trivalent de Sorex.

3. Hétérochromatie des chromosomes sexuels.

Comme chez les Chiroptères, les chromosomes $X$ et $Y$ sont toujours hétérochromatiques aux stades pachytène et diffus. A la 
diacinèse, ce phénomène subsiste, chez Crocidura russula, mais dans une faible mesure, et pas constamment. A la métaphase de la première division de maturation, le bivalent $X Y$ est hétérochromatique chez Talpa, Neomys et Crocidura. C'est en général le segment différentiel de l' $X$ qui est plus coloré que les autosomes, ainsi que l' $Y$. Chez Crocidura, on observe une hétérochromatie réversible, qui affecte d'abord le segment différentiel, puis, à 'anaphase, le segment pair. L'hétérochromatie ne dure que jusqu'à la télophase de la première division, et ne se maintient pas, comme 'est le cas chez Myotis myotis par exemple, jusqu'à la fin de la seconde division.

\section{Evolution chromosomique chez les Insectivores.}

Bien que le nombre des espèces étudiées jusqu'à maintenant soit trop faible pour autoriser des conclusions définitives, il est déjà clair que l'ordre des Insectivores est beaucoup moins homogène au point de vue cytologique que celui des Chiroptères, puisqu'à l'intérieur d'une même famille, les Soricidae, par exemple, les nombres fondamentaux varient de 44 (Sorex araneus) à 92 (Neomys fodiens). En tenant compte de tous les travaux, on peut établir le tableau suivant:

\begin{tabular}{|c|c|c|c|c|}
\hline Espèces & $2 \mathrm{~N}$. & $\begin{array}{c}\text { Nombre } \\
\text { de V. }\end{array}$ & N. F. & Auteur \\
\hline $\begin{array}{l}\text { ERINACEIDAE } \\
\text { Erinaceus europaeus L. } \\
\text { TALPIDAE } \\
\text { Talpa europaea L. } \\
\text { Mogera insularis Swinhoe } \\
\text { SoricidaE }\end{array}$ & $\begin{array}{l}52 \\
42 \\
40 \\
23\end{array}$ & $\begin{array}{c}40 \\
7-8 \\
6 \\
21\end{array}$ & $\begin{array}{c}87 \\
67 \\
58 \\
\\
92 \\
49-50 \\
46 \\
44\end{array}$ & $\begin{array}{l}\text { Bovey, } 1949 \\
\text { Bovey, } 1949 \\
\text { TAteishi, } 1938 \\
\text { Bovey, } 1949 \\
\text { Bovey, } 1949 \\
\text { TATEISHi, } 1938 \\
\text { Bovey, } 1948\end{array}$ \\
\hline
\end{tabular}

Sorex araneus peut être rapproché des Crocidures, tandis que, par son nombre fondamental élevé, Neomys fodiens évoque plutôt 
Talpa ou Erinaceus. La loi de Robertson est ici en défaut, et le seul groupe où l'on puisse envisager son application approximative est celui des Soricidae, Neomys étant mis à part.

Les Insectivores sont unanimement considérés comme un ordre très ancien, et semblent avoir existé dès la période crétacée, mais les formes fossiles très primitives sont plus apparentées aux Centetes de Madagascar qu'aux Insectivores actuels d'Europe. Très abondants à l'Eocène, ils représentent aujourd'hui les derniers restes d'un groupe presque éteint, qui fut probablement, à l'origine, la souche d'où se détachèrent de nombreux ordres de Mammifères, notamment les Chiroptères et les Primates (A.-S. Roмer, 1947). Parmi les Insectivores, les Erinaceidae représentent le groupe le plus primitif, dont dérivent les Talpidae, et, plus récemment, les Soricidae, considérés par Romer comme moins primitifs que les Talpidae.

La cytologie peut-elle infirmer ou confirmer ces hypothèses? Nous nous étions demandé, en étudiant le même problème chez les Chiroptères, si l'évolution chromosomique s'était faite avec prédominance des fusions centriques ou au contraire des fragmentations d'éléments métacentriques donnant naissance à des acrocentriques. La question n'avait pu être résolue, faute de documents paléontologiques et cytologiques assez abondants. La situation est encore moins favorable en ce qui concerne les Insectivores, et les tentatives de connaître le sens de cette évolution aboutissent à des contradictions.

La parenté avec les Primates ne peut être examinée, ces derniers, à part l'Homme, étant trop peu connus au point de vue cytologique. Remarquons cependant que l'Homme, avec son grand nombre de chromosomes en $V$ et son nombre fondamental voisin de 80 , possède un assortiment chromosomique fort comparable à celui du Hérisson $(N . F .=87)$, comme l'avait déjà relevé Painter (1925).

Les affinités entre les Chiroptères et les Insectivores se manifestent si l'on compare entre eux les Talpidae, les Erinaceidae, et Neomys, d'une part, les Pteropidae, les Nycteridae, d'autre part. Chez tous ces animaux, le nombre fondamental est élevé, et les chromosomes métacentriques nombreux. En outre, les chromosomes sexuels sont du même type et ont la même forme dans les deux ordres. 
Quoi qu'il en soit, la diversité des conditions cytologiques à l'intérieur de l'ordre des Insectivores illustre bien le caractère disparate et quelque peu artificiel de ce groupe systématique. Il est regrettable que les représentants les plus primitifs de cet ordre, les Chrysochloridae et les Centedidae n'aient pas encore fait l'objet d'une étude cytologique. Lorsque nous connaîtrons les formules chromosomiques de nombreuses espèces appartenant à ces diverses branches, il sera alors possible d'émettre une hypothèse sur leurs relations phylétiques, ce qui, pour l'instant, serait certainement prématuré.

\section{CONCLUSIONS GÉNÉRALES}

Loin d'être fastidieuse, l'étude cytologique systématique d'un groupe d'animaux peut aboutir à la découverte de faits cntièrement nouveaux. L'utilité de telles recherches apparaît clairement si l'on considère la faible proportion d'espèces étudiées à ce point de vue, par rapport à l'ensemble du règne animal. C'est justement cet aspect trop lacunaire de nos connaissances dans ce domaine qui rend si difficiles toute comparaison et toute généralisation.

De l'étude des deux ordres des Chiroptères et des Insectivores, nous pouvons tirer les conclusions suivantes:

1. Les Chiroptères sont beaucoup plus homogènes que les Insectivores.

2. Les nombres fondamentaux, comme les nombres diploïdes, paraissent sans rapport entre les deux ordres. On peut toutefois établir quelques analogies entre les Erinaceidae, les Talpidae, les Pteropidae et les Nycteridae, familles qui sont toutes considérées comme très primitives.

3. Les chromosomes sexuels des Chiroptères et des Insectivores montrent en revanche une remarquable uniformité: morphologie identique, constance de la pré-réduction, hétérochromatie positive, insertion médiane ou sub-médiane du centromère de l' $X$, sont autant de caractères communs, qui témoignent des relations phylétiques existant entre les deux ordres. 


\section{RÉSUMÉ}

1. J'ai étudié treize espèces de Chiroptères et cinq espèces d'Insectivores. Les résultats obtenus se résument comme suit:

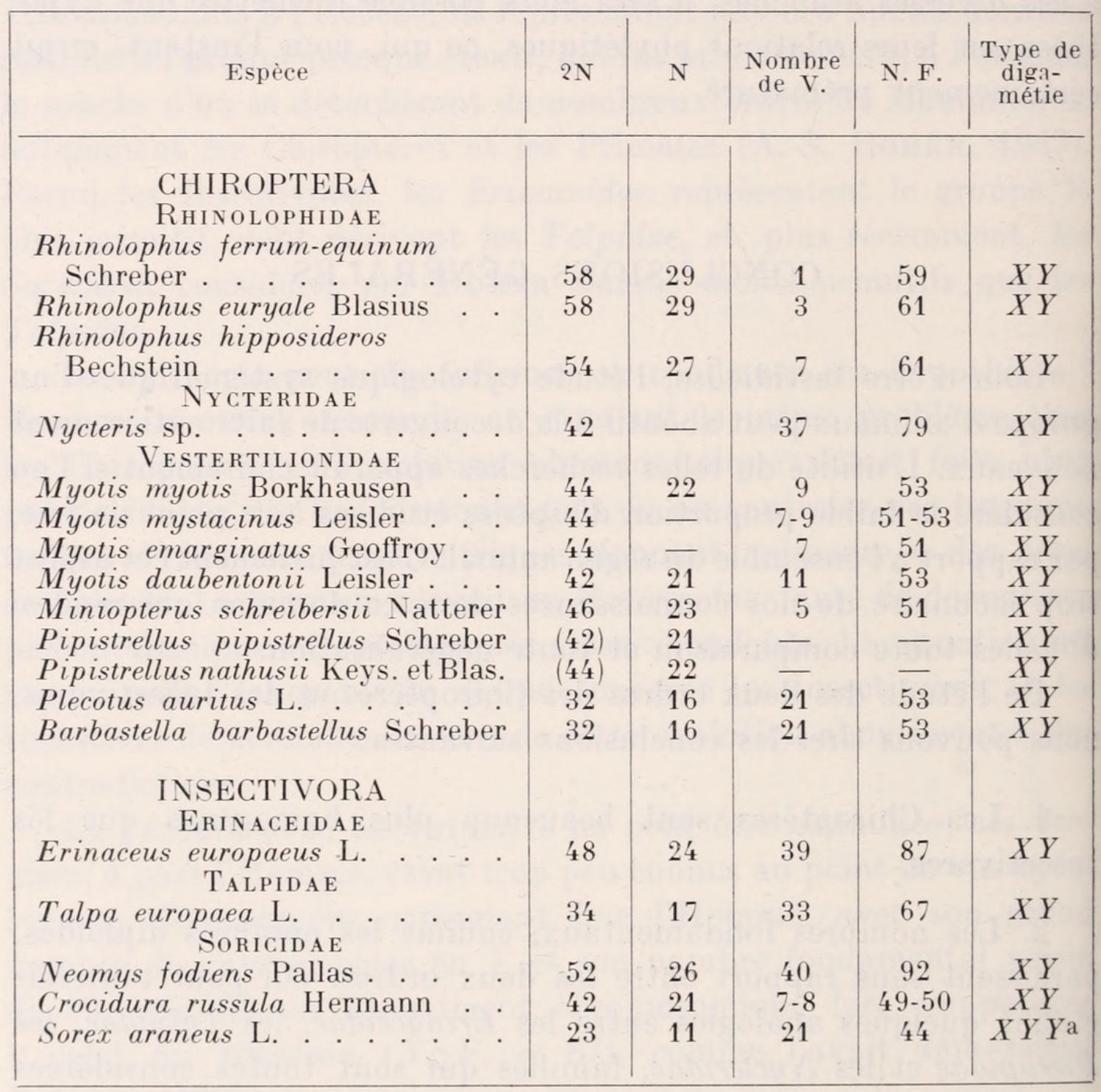

2. La digamétie est du type $X Y$ chez les mâles de toutes les espèces étudiées, sauf Sorex araneus L., qui possède un trivalent sexuel $X-Y-Y a$, dont la description est donnée.

3. Durant les stades pré-méiotiques, les hétérochromosomes évoluent tout d'abord dans une vésicule hétérocaryotique. La nature de leurs liaisons est discutée. 
4. Il y a toujours pré-réduction chez les Insectivores, et, probablement, chez les Chiroptères.

5. Chez la plupart des espèces étudiées, les chromosomes sexuels ont à la méiose une hétérochromatie positive.

6. La loi de Robertson trouve chez les Chiroptères une excellente justification. Par contre, elle ne peut s'appliquer aux formules très peu homogènes des Insectivores, dont les affinités phylétiques ne sauraient être, pour l'instant, précisées par la cytologie.

7. Les affinités entre les Chiroptères et les Insectivores, peu marquées si l'on analyse les formules chromosomiques, sont plus nettes lorsqu'on compare les chromosomes sexuels.

8. Le trivalent sexuel de Sorex araneus L. représente un cas absolument nouveau chez les Vertébrés. Il dérive probablement d'un couple $X Y$ normal par une translocation sur l' $X$ d'un segment autosomique.

\section{AUTEURS CITÉS}

1923. Agar, W. E. The male meiotic phase in two genera of marsupials (Macropus and Petauroides). Quart. Journ. Micr. Sc., 67, p. 183. 1904. Andersen, K. et Matschie, P. Übersicht einiger geographischen Formen der Untergattung Euryalus. SB. Ges. Naturfr. Berlin, 5, p. 71.

1912. Aтнias, M. Sur les divisions de maturation de l'œuf des Mammifères. Arch. Inst. Bacter. Cam. Pest., Lisbonne, 3, p. 287.

1935. Boule, M. et Piveteau, J. Les fossiles. Paris.

1948. Bovey, R. Un type nouveau d'hétérochromosomes chez un Mammifère: le trivalent sexuel de Sorex araneus L. Arch. d. Jul. KlausStift., 23, p. 506.

1949. _ La formule chromosomique de quelques Insectivores indigènes. Experientia, 5, p. 72.

1927. Courrier, R. Etude sur le déterminisme des caractères sexuels secondaires chez quelques Mammifères à activité testiculaire périodique. Thèse, Liége. Arch. de Biol., 37, p. 173.

1937. Darlington, C. D. Recent advances in Cytology. 2nd ed., London. 1917. Hance, R. T. The fixation of mammalian chromosomes. Anat. Rec., 12 , p. 371. 
1935. Heberer, G. Die Ergebnisse der Chromosomenforschung beim Menschen. Zeitschr. f. Mensch. Vererb. und Konstitutionslehre, 19 , p. 96.

1912. Jordan, H. E. Notes on the spermatogenesis of the bat. Anat. Anz., 40 , p. 513.

1934. Koller, P. C. et Darlington, C. The genetical and mechanical properties of the sex-chromosomes. I. Rattus norvegicus, $\hat{\sigma}$. Journ. Genet., 29, p. 159.

1936. Koller, P. C. Chromosome behaviour in the male ferret and mole during anoestrus. Proc. Roy. Soc., London, Ser. B, 121, p. 192.

1941. Makino, S. Studies on the Murine Chromosomes. I. Cytological investigations of Mice, inclued in the genus Mus. Jour. Fac. Sc. Hokk. Imp. Univers. Ser. VI, 7, p. 305.

1948. A study of the chromosomes in two species of bats (Chiroptera). Biol. Bull., 94, p. 275.

1931. Matthey, R. Chromosomes de Reptiles Sauriens, Ophidiens et Chéloniens. L'évolution de la formule chromosomiale chez les Sauriens. Rev. suisse Zool., 38, p. 117.

1932. - Les chromosomes et la systématique zoologique. Rev. suisse Zool., 3.9, p. 229.

1936. - Le problème des hétérochromosomes chez les Mammifères. Arch. Biol., 47, p. 319.

1938. - Contribution nouvelle à l'étude des hétérochromosomes chez les Mammifères, et singulièrement chez les Rongeurs. Journ. Genet., 36, p. 73.

1945. L'évolution de la formule chromosomiale chez les Vertébrés. Experientia, 1, p. 50.

1947. — Encore les hétérochromosomes des Apodemus. Arch. d. Jul. Klaus-Stift., 22, p. 85.

1949 a. Chromosomes sexuels géants chez un Campagnol, Microtus agrestis L. Experientia, 5, p. 72.

1949 b. Les chromosomes des Vertébrés. Rouge, Lausanne.

1948. - et Bover, R. La formule chromosomique chez cinq espèces de Chiroptères. Experientia, 4, p. 1.

1928. Minouchi, O. On the fixation of chromosomes in mammals and some others animals. Jap. Journ. Zool., 1, p. 219.

1928. - Spermatogenesis of the albino rat (Mus norvegicus albus). Jap. Journ. Zool., 1, p. 255.

1937. Oguma, K. Absence of the Y-chromosome in the vole, Microtus montebelli Edw., with supplementary remarks on the sex-chromosomes of Evotomys and Apodemus. Cytologia Fuj. Jub. vol., p. 796.

1937. Oguma, K. et Makino, S. A new list of the chromosome numbers in Vertebrata. Journ. Fac. Sc. Hokkaido Univ., S. VI, 5.

1925. Painter, Th. S. A comparative study of the chromosomes of mammals. Amer. Nat., 59, p. 385. 
1943. Ponteconvo, G. Meiosis in the striped hamster (Cricetulus griseus Milne-Edw.) and the problem of heterochromatin in mammalian sex-chromosomes. Proc. Roy. Soc. Edinburgh, Sect. B, 62, p. 32.

1938. Renaud, P. La formule chromosomiale chez sept espèces de Muscardinidae et de Microtinae indigènes. Rev. suisse Zool., 45, p. 349. 1917-1922. Revilliod, P. Contribution à l'étude des Chiroptères des terrains tertiaires. Mém. Soc. paléont. suisse, 43, 44, 45.

1916. Robertson, W. R. B. Chromosome studies. Journ. Morph., $2 \%$. 1947. Romer, A. S. Vertebrate Paleontology. 3rd impr., Chicago.

1947. Ryberg, O. Studies on bats and bat parasites. Stockholm.

1932. Shiwago, P. I. et Andres, A. H. Die Geschlechtschromosomen des Menschen. Z. Zellforsch., 16.

1936. Tateishi, S. The chromosomes of the Formosan Hare, Lepus formosus and of the Rabbit, Lepus cuninculus. Zool. Mag. (Japan), 48, p. 617.

1937. — Zool. Mag. (Japan), 49, p. 1. (Titre en japonais.)

1938. - The chromosomes of two species of Insectivora. Annotationes Zoologicae Japonenses, 17, p. 515.

1910. Van der Stricht, O. La structure de l'œuf des mammifères. Mem. Acad. roy. Belgique (Sci.), Ser. 2, t. 2, No. 2, p. 1.

1928. Weber, M. Die Säugetiere, 2. Aufl. Iena.

1945. White, M. J. D. Animal cytology and evolution. Cambridge. 1925. Wilson, E. B. The cell in development and heredity, 3rd ed., New-York. 


\section{$2 \mathrm{BHL}$ Biodiversity Heritage Library}

Bovey,

Rene

. 1949. "Les chromosomes des Chiroptères et des Insectivores." Revue suisse de zoologie 56, 371-459. https://doi.org/10.5962/bhl.part.117900.

View This Item Online: https://www.biodiversitylibrary.org/item/148885

DOI: https://doi.org/10.5962/bhl.part.117900

Permalink: https://www.biodiversitylibrary.org/partpdf/117900

\section{Holding Institution}

American Museum of Natural History Library

\section{Sponsored by}

BHL-SIL-FEDLINK

\section{Copyright \& Reuse}

Copyright Status: In copyright. Digitized with the permission of the rights holder.

Rights Holder: Muséum d'histoire naturelle - Ville de Genève

This document was created from content at the Biodiversity Heritage Library, the world's largest open access digital library for biodiversity literature and archives. Visit BHL at https://www.biodiversitylibrary.org. 